University of Nebraska - Lincoln

DigitalCommons@University of Nebraska - Lincoln

2-26-2021

\title{
The influence of juvenile dinosaurs on community structure and diversity
}

Katlin Schroeder

University of New Mexico, maguire@unm.edu

S. Kathleen Lyons

University of Nebraska - Lincoln, katelyons@unl.edu

Felisa A. Smith

University of New Mexico, fasmith@unm.edu

Follow this and additional works at: https://digitalcommons.unl.edu/bioscifacpub

Part of the Biology Commons, Paleontology Commons, and the Population Biology Commons

Schroeder, Katlin; Lyons, S. Kathleen; and Smith, Felisa A., "The influence of juvenile dinosaurs on community structure and diversity" (2021). Faculty Publications in the Biological Sciences. 857. https://digitalcommons.unl.edu/bioscifacpub/857

This Article is brought to you for free and open access by the Papers in the Biological Sciences at DigitalCommons@University of Nebraska - Lincoln. It has been accepted for inclusion in Faculty Publications in the Biological Sciences by an authorized administrator of DigitalCommons@University of Nebraska - Lincoln. 


\title{
The influence of juvenile dinosaurs on community structure and diversity
}

\author{
Katlin Schroeder, ${ }^{1}$ S. Kathleen Lyons, ${ }^{2}$ and Felisa A. Smith ${ }^{1}$ \\ 1 Department of Biology, University of New Mexico, Albuquerque, NM 87131, USA \\ 2 School of Biological Sciences, University of Nebraska-Lincoln, Lincoln, NE 68588, USA \\ Corresponding author — K. Schroeder, email: maguire@unm.edu
}

\begin{abstract}
Despite dominating biodiversity in the Mesozoic, dinosaurs were not speciose. Oviparity constrained even gigantic dinosaurs to less than $15 \mathrm{~kg}$ at birth; growth through multiple morphologies led to the consumption of different resources at each stage. Such disparity between neonates and adults could have influenced the structure and diversity of dinosaur communities. Here, we quantified this effect for 43 communities across 136 million years and seven continents. We found that megatheropods (more than $1000 \mathrm{~kg}$ ) such as tyrannosaurs had specific effects on dinosaur community structure. Although herbivores spanned the body size range, communities with megatheropods lacked carnivores weighing 100 to $1000 \mathrm{~kg}$. We demonstrate that juvenile megatheropods likely filled the mesocarnivore niche, resulting in reduced overall taxonomic diversity. The consistency of this pattern suggests that ontogenetic niche shift was an important factor in generating dinosaur community structure and diversity.
\end{abstract}

Dinosaurs were the dominant terrestrial vertebrates for $>150$ million years, yet their species diversity, particularly at sizes $<60 \mathrm{~kg}$, remained well below that of other fossil groups (1). Moreover, their overall body

Published in Science 371, 941-944 (2021).

DOI: $10.1126 /$ science.abd9220

Copyright (C) 2021 Katlin Schroeder, S. Kathleen Lyons, and Felisa A. Smith; published by the

American Association for the Advancement of Science. Used by permission. Submitted 20 July 2020; accepted 21 January 2021; published 26 February 2021. 
size distribution differed from other vertebrates. Because small-bodied vertebrates can finely partition resources and have high turnover between environments $(2,3)$, they typically have the highest diversity across regions. Yet curiously, large-bodied dinosaurs were the most diverse. This was particularly true for herbivorous sauropods and ornithischians, whereas the predominantly carnivorous theropods exhibited a more uniform range of sizes globally (4). Although the preponderance of large-bodied forms may be partially due to taphonomy (5), some $90 \%$ of dinosaur species $<60 \mathrm{~kg}$ would have to be missing from the fossil record for the body mass distribution of dinosaurs to resemble that of extinct mammals, which display a pattern less skewed by size (4). Rather, dinosaurs' global body mass distribution patterns may have been linked to their physiology; as oviparous organisms, the largest dinosaurs grew from disproportionately small infants (6). Many dinosaurs exhibited marked morphological differences between juveniles and adults $(7,8)$, resulting in the utilization of different resources through growth and development (9-12), a relatively rare terrestrial life history strategy observed mostly in large egg-laying reptiles (13). Moreover, rapid growth combined with low adult survivorship (14-16) resulted in large populations of juvenile dinosaurs (17) that may have competed with dinosaurs that were small and medium-sized as adults.

Here, we tested whether low dinosaur species diversity and their unusual body size distribution was, at least partially, due to the large disparity between neonate and adult body size, with juveniles of largerbodied species filling ecological niches that might have otherwise been available to other taxa. This concept of "ontogenetic niche shift" (ONS) in dinosaurs is widely assumed based on modern correlates $(10,13$, $14,18,19)$. For example, based on modeling of hypothetical dinosaur communities, Codron et al. $(9,11)$ predicted that ONS led to reduced diversity of dinosaurs weighing between 1 and $1000 \mathrm{~kg}$. Despite these predictions, little work has empirically explored juveniles' influence on community structure and overall dinosaur diversity $(11,19)$. Thus, we examined small-scale body size patterns for evidence of competitive interactions using fossil evidence from dozens of communities representing a wide variety of environments spanning most of dinosaur evolution and evaluated the potential effects of spatial scale and trophic affiliation using well-constrained groups of biologically interacting species. 
Our analyses are based on 43 dinosaur communities constructed from data extracted from the Paleobiology Database $(20,21)$ (Table S1). From this baseline, each species' occurrence and taxonomic validity were checked individually against the literature, with taxa deemed synonymous by most experts removed and new taxa absent from the Paleobiology Database added. Masses were derived using averages from the primary literature (Table S1). Wherever possible, formations were limited to smaller subsets of co-occurring species. Our dataset represents seven continents spanning 136 million years and includes $>550$ species. We predicted that dinosaur communities with strong local drivers would diverge from the global distribution $(2,22,23)$. Because ecological interactions such as competition might not have influenced carnivorous and herbivorous dinosaurs equally (24), the shape of each carnivore and herbivore dinosaur guild within each community was compared against the global distribution (4).

We found that the overall body size distributions within communities were consistently bimodal regardless of continent, taxa, and time, resulting in less-extreme skew toward large size than evidenced in the global distribution (global skew $=-0.577$, community average skew $=-0.365$; Table S2). The disparity between the local and global distributions was driven primarily by small (10 to $100 \mathrm{~kg}$ ) carnivorous dinosaurs (Table S4 and Fig. 1); when examined separately, local herbivore body mass distributions closely reflect their global distribution, suggesting that ecological interactions have little effect on their distribution $[\mathrm{P}<0.05$ in $40 \%$ of communities, Kolmogorov-Smirnov (K-S) test; Table S3].

By contrast, most carnivorous guilds within communities differed from the global carnivore pattern ( $\mathrm{P}<0.05$ for $64 \%$ of communities, K-S test; Table S4), as predicted for strong local interactions. Pairwise comparisons between carnivore guild distributions were nonsignificant in 92\% of tests ( $\alpha=0.05$, two-tailed t test with Bonferroni correction for multiple comparisons: $\alpha=0.000058$ nonsignificant in 99.7\%, Table S7) despite differing variances, means, and sample sizes, suggesting similar underlying drivers across communities. The only exceptions are formations lacking megatheropods (carnivores $>1000 \mathrm{~kg}$ ) (e.g., Tremp and Bissekty), those dominated by very small taxa (e.g., Yixian), or those containing multiple sauropods (e.g., Morrison and Lameta), where the availability of multiple enormous prey species may have reduced interspecific competition and allowed the coexistence of an unusually diverse assortment of carnivores. 


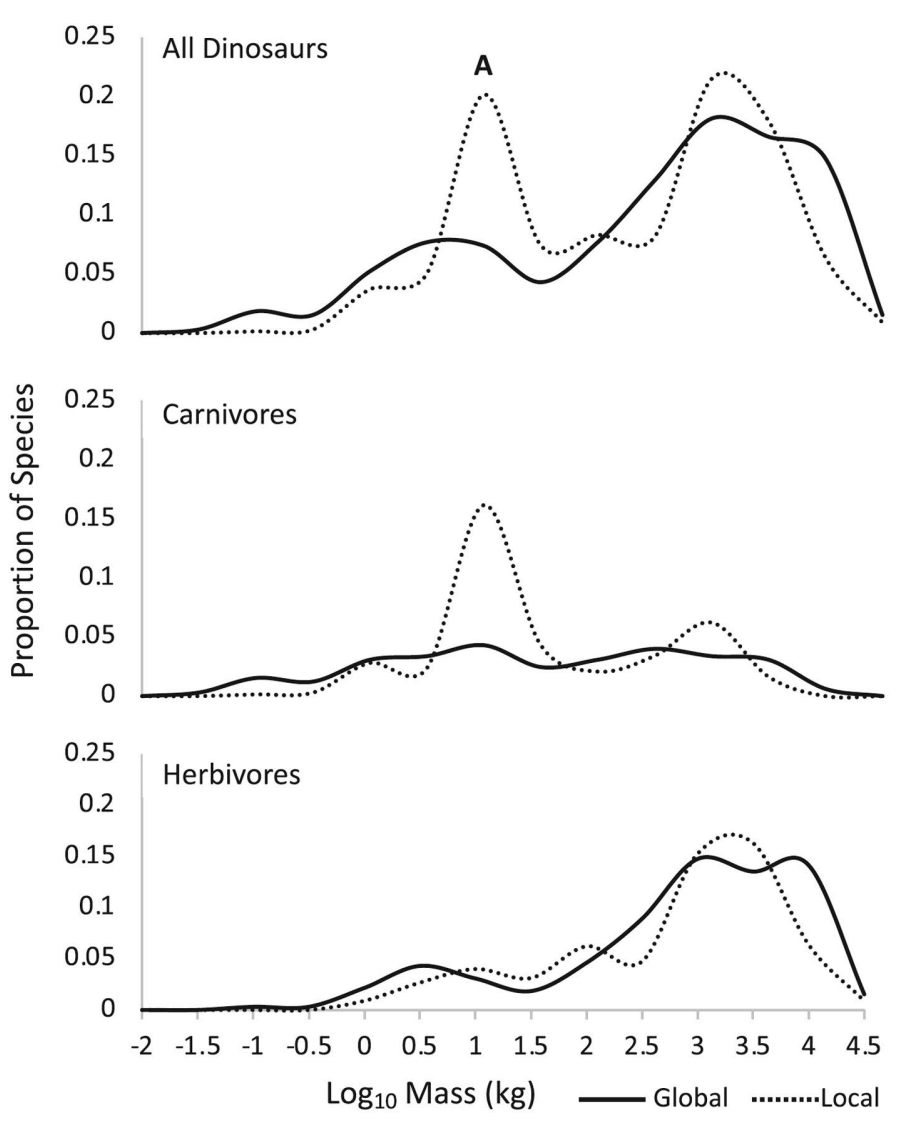

Fig. 1. Community divergence from global distributions. Distributions comparing 1303 global taxa with local community taxa (median). Overall, global taxa are more left skewed and communities are more bimodal. "A" indicates the largest deviation from the global distribution; the same deviation is clearly shown in the carnivore distribution.

Community distributions exhibited a persistent lack of carnivorous dinosaurs weighing between 100 and $1000 \mathrm{~kg}$ (Fig. 2). The least likely body size of carnivorous taxa was consistently in the 100 - to $300-\mathrm{kg}$ range (Fig. S1). For perspective, if the modern mammal carnivore assemblage of Kruger National Park were similarly structured, there would be no carnivores between the size of an African lion $(190 \mathrm{~kg})$ and a bateared fox (4 kg) (Fig. 3). The carnivore "gap" was above the expected limit of taphonomic size bias against small dinosaurs (5), and the drivers of such bias were unlikely to have selectively affected carnivores but not herbivores, suggesting that the gap represents a true biological signal. Moreover, it is unlikely that other clades such as mammals or crocodylomorphs occupied this body size niche because no known Mesozoic mammals exceeded $15 \mathrm{~kg}(22)$, and crocodylomorphs were predominantly 


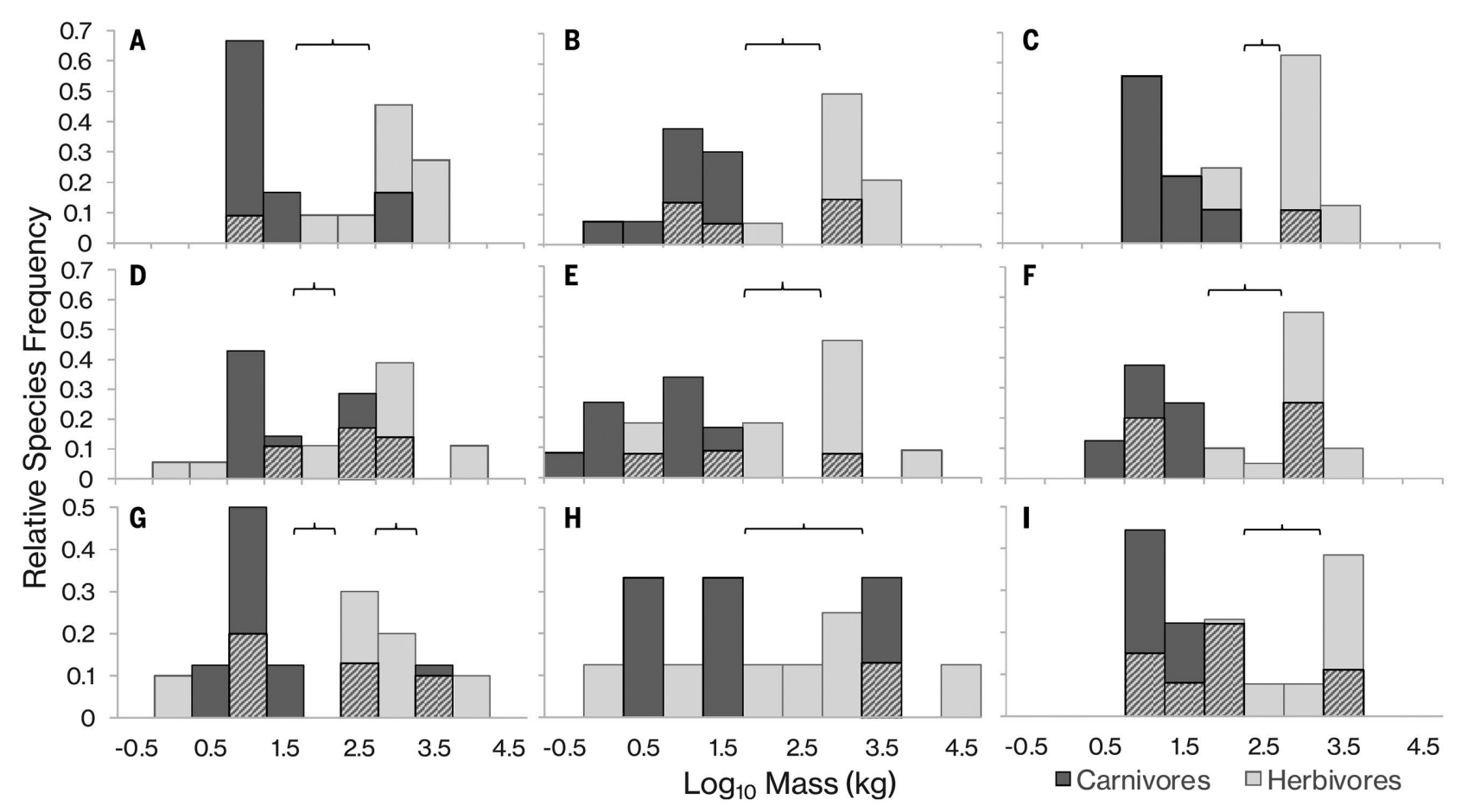

Fig. 2. Community mass-species distributions of nine formations. The formations shown are as follows: (A) Judith River, (B) Dinosaur Park, (C) Two Medicine, (D) Bayan Shireh, (E) Barun Goyot, (F) Horseshoe Canyon, (G) Cedar Mountain, (H) Cloverly, (I) Hell Creek. Brackets illustrate the gaps in carnivore distributions.

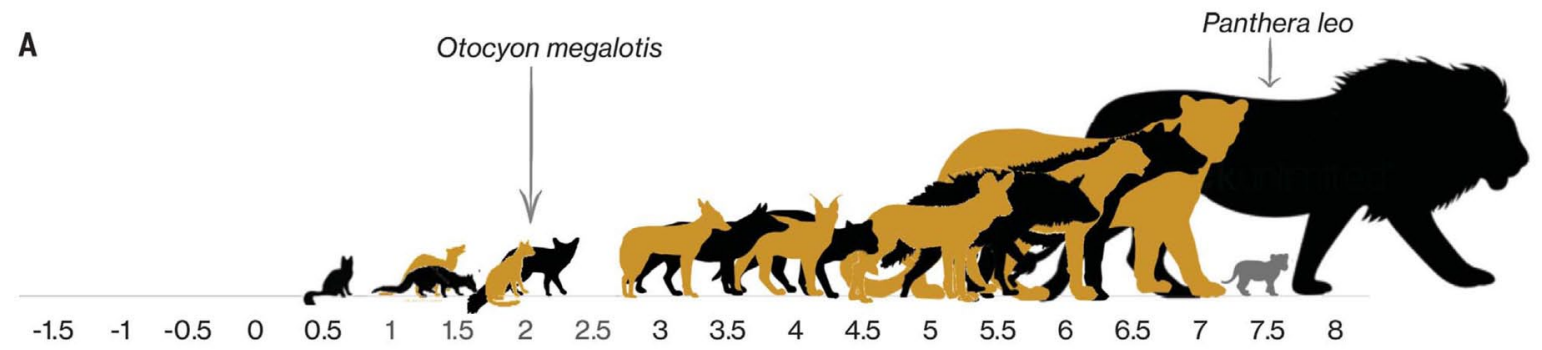

B

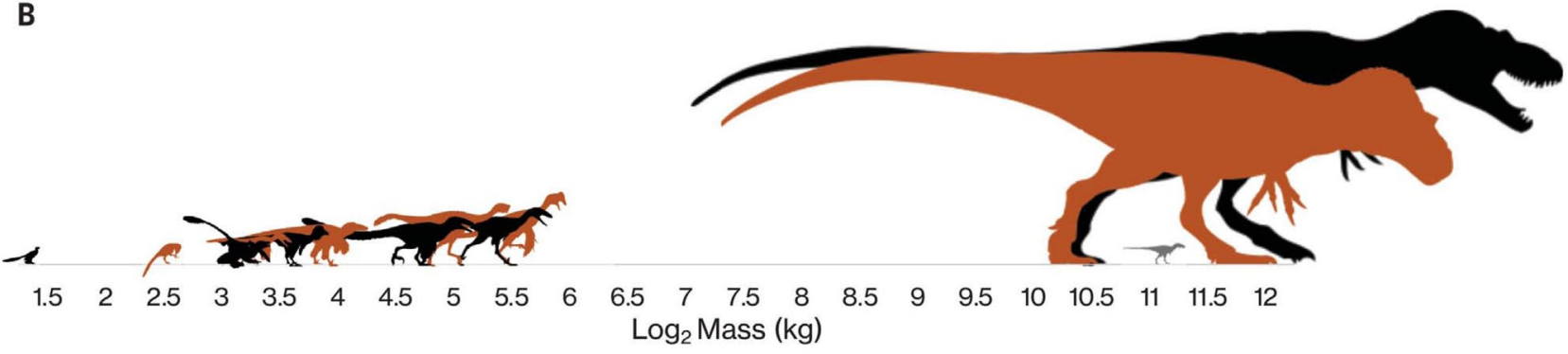

Fig. 3. The dinosaur gap versus modern carnivorous mammals. (A) Carnivorous mammals of Kruger National Park organized to scale by mass. (B) Carnivorous dinosaurs of Dinosaur Park Formation if the largest carnivore were scaled equally to the largest mammalian carnivore in Kruger. Infants (gray) of the largest species shown below adult to show relative growth requirement. 
semiaquatic after the Triassic (25). Furthermore, the width of the carnivore body size gap is correlated with the size of the largest carnivore (Kendall rank $\mathrm{t}=0.437, \mathrm{P}=0.000652$ ). The presence of megatheropods in the community decreased the likelihood of co-occurring species between 100 and $1000 \mathrm{~kg}$ even further (Table S5). Formations without megatheropods, such as Yixian Lujiantun, did not exhibit body size discontinuities in their carnivorous dinosaur assemblages.

Although the overall distribution of carnivore body size was consistent, the gap itself was dynamic. From the Jurassic to the Cretaceous, the size gap in carnivore species shifted toward larger sizes, mirroring the evolutionary increase in overall dinosaur size (26), and widened from an average of $436 \mathrm{~kg}$ to $>2060 \mathrm{~kg}$. We suspect that the shift and expansion of the body size gap was caused by a number of changes from the Jurassic to the Cretaceous resulting in increased competition, including (i) decrease by half of average prey body mass, limiting the potential for size partitioning (26); (ii) the diversification of small, potentially endothermic carnivorous dinosaurs (27); and (iii) heightened ONS in Cretaceous megatheropods.

A smaller size gap was found in Jurassic communities, which were characterized by multiple large allosauroids and medium-sized ceratosaurs. Allosauroidea was a morphologically diverse clade (28), which likely facilitated the co-occurrence of multiple carnivores within communities. Juvenile allosaurs were more similar to adults than Cretaceous megatheropods (29), resulting in fewer feeding niche shifts through ontogeny. Predation on sauropods (30) may have reduced allosaurs' competition with ceratosaurs, which have been associated with piscivory or omnivory, respectively (31). This relatively high morphological differentiation and associated dietary niche partitioning combined with limited ONS in megatheropods may have allowed for the coexistence of largeand medium-sized Jurassic carnivores.

The end of the Jurassic saw a drastic reduction in the diversity of both sauropods and stegosaurs and may have led to the disappearance of many allosauroid taxa (32). Replacing the diverse megatheropod guilds of the Jurassic were Cretaceous communities dominated by a single clade: tyrannosaurs in the north and abelisaurs in the south. Both tyrannosaurs and abelisaurs have been associated with extensive morphological changes through ontogeny $(7,33)$. Concurrent diversification of dromaeosaurs added competitive pressure on the truncated prey base 
(34). The ornithischian prey that replaced sauropods likely traveled in multigenerational herds (35), limiting the possibility of predation of isolated juveniles. We suggest that competition for a limited prey source by both large and small carnivores, and the broadening of megatheropod niches, resulted in a widening of the carnivore gap.

For juvenile megatheropods to exclude smaller species from the community, they must represent a non-negligible proportion $(>50 \%)$ of the biomass. Moreover, juvenile peak biomass must fall predominantly within the carnivore gap. To evaluate the effect of juveniles $[<16$ years of age $(12,33)]$ on community composition, we calculated the proportion of juvenile biomass using published growth rates derived from lines of arrested growth and survivorship curves based on relative age abundance from mass-death assemblages recorded in the fossil record (17, 36-38).

We calculated biomass through ontogeny for 1000 individual cohorts of six tyrannosaurs and four allosaurs by multiplying the mass $\left(\mathrm{M}_{\mathrm{a}}\right)$ and survivorship $\left(S_{a}\right)$ at age in years (a) such that the proportion of any species' biomass represented by juveniles $\left(\mathrm{BM}_{\mathrm{J}}\right)$ is as follows:

$$
\begin{aligned}
\mathrm{BM}_{\mathrm{J}}=\sum & \left(\frac{\mathrm{M}_{1} * \mathrm{~S}_{1}}{\sum\left(\mathrm{M}_{1} * \mathrm{~S}_{1}, \mathrm{M}_{2} * \mathrm{~S}_{2}, \ldots \mathrm{M}_{\max } * \mathrm{~S}_{\max }\right)}\right), \\
& \left(\frac{\mathrm{M}_{2} * \mathrm{~S}_{2}}{\sum\left(\mathrm{M}_{1} * \mathrm{~S}_{1}, \ldots \mathrm{M}_{\max } * \mathrm{~S}_{\max }\right)}\right) \ldots \\
& \left(\frac{\mathrm{M}_{16} * \mathrm{~S}_{16}}{\sum\left(\mathrm{M}_{1} * \mathrm{~S}_{1}, \ldots \mathrm{M}_{\text {max }} * \mathrm{~S}_{\max }\right)}\right)
\end{aligned}
$$

We then related $\mathrm{BM}_{\mathrm{J}}$ to the proportion of mass contained in adults, set to 1 , so that relative juvenile species proportion $\left(\mathrm{RSP}_{\mathrm{J}}\right)$ is equal to the following:

$$
\mathrm{RSP}_{\mathrm{J}}=\mathrm{BM}_{\mathrm{J}} /\left(1-\mathrm{BM}_{\mathrm{J}}\right)
$$

For example, if juveniles represented $60 \%$ of the biomass of Tyrannosaurus rex, then the juvenile "morphospecies" would be equivalent to $1.5 \times$ the taxonomic species. Tyrannosaurs and other megatheropods did not live long past somatic maturity (16), and their juvenile growth rate 


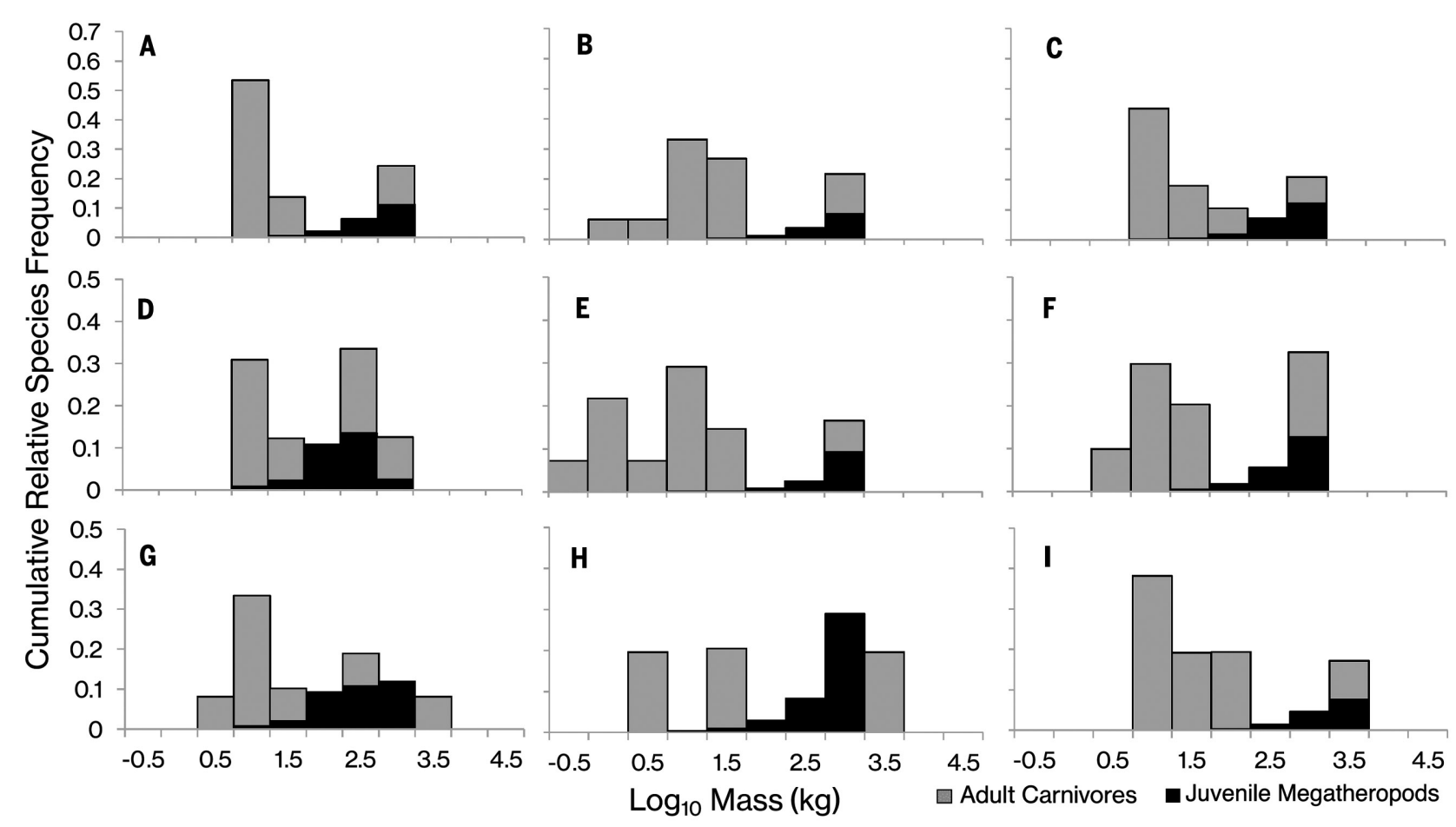

Fig. 4. Community mass-species distributions with juvenile megatheropods as morphospecies stacked with adult conspecifics. The formations shown are as follows: (A) Judith River, (B) Dinosaur Park, (C) Two Medicine, (D) Bayan Shireh, (E) Barun Goyot, (F) Horseshoe Canyon, (G) Cedar Mountain, (H) Cloverly, (I) Hell Creek. The influence of juveniles was highest within the carnivore gap and was proportional to at least $60 \%$ of adults in all measured communities. Megatheropods $<3000 \mathrm{~kg}$ exerted the most influence, matching or outweighing their adult conspecifics in more than half of the measured formations.

approached that of mammals and birds (12). This supports our finding that megatheropod biomass peaked at sexual maturity (16 to 19 years of age) and followed a log-normal distribution (Fig. S2). For all 10 species examined, juvenile biomass was proportional to least $60 \%$ of adult conspecifics (Table S6) and exceeded adult biomass in five tyrannosaur species. Substantial proportions of juvenile biomass, including peak biomass, fell within the range of the size gap in all communities (Fig. 4). Thus, juvenile megatheropods represented taxonomically identical but ecologically disparate morphospecies within their communities, with the greatest potential influence in the mass range of 300 to $1000 \mathrm{~kg}$. Our results support the hypothesis that juvenile megatheropods effectively filled the niche of medium-sized carnivores, or mesocarnivores 
and therefore likely limited diversification of theropods with adult body sizes that fell within this range.

That large carnivorous dinosaurs may have filled multiple niches through ontogeny is not a new assertion $(7,9,11,12,32)$, yet despite their morphological disparity, adults and juveniles continue to be grouped together in diversity indices, which is accurate taxonomically but not ecologically. Our analysis demonstrates the influence that juvenile megatheropods would have had as morphospecies on their community. We found a gap in the community body size distribution of carnivorous dinosaurs regardless of continent, biome, formation size, or species examined. Our analysis demonstrates that this gap was likely filled by juvenile megatheropods and suggests that low taxonomic diversity in carnivorous dinosaurs was not caused solely by taphonomy or collection bias but rather by competition for resources within and among body size niches filled by juveniles. Dinosaurs existed in a specialized terrestrial community structure, largely organized as a result of their extreme size, ovipary, and resulting ontogenetic niche shift. The "grow fast, die young" approach of megatheropods resulted in a predominance of juveniles in communities, filling the morphological and functional role of mesocarnivores, which as a result are absent from the fossil record as individual species, artificially deflating diversity indices of dinosaurs as a whole.

Acknowledgments: We thank A. Kooser and S. Schappert for their feedback on this manuscript and invaluable advice on figures.

Funding: None.

Author contributions: K.S., S.K.L., and F.A.S. conceived and designed the experiments and wrote the manuscript. K.S. performed the experiments, analyzed the data, and contributed reagents, materials, and analysis tools.

Competing interests: The authors declare no competing interests.

Data and materials availability: All data are available in the main text or supplementary materials.

\section{Supplementary materials attached to this archive record:}

Materials and Methods

Figs. S1 and S2

Tables S1 to S7

References (39-378)

MDAR Reproducibility Checklist 


\section{References}

1. S. C. Wang, P. Dodson, Proc. Natl. Acad. Sci. U.S.A. 103, 13601-13605 (2006).

2. J. H. Brown, P. F. Nicoletto, Am. Nat. 138, 1478-1512 (1991).

3. D. A. Kelt et al., Ecology 77, 746-761 (1996).

4. E. J. O'Gorman, D. W. E. Hone, PLOS ONE 7, e51925 (2012).

5. C. M. Brown, D. C. Evans, N. E. Campione, L. J. O'Brien, D. A. Eberth, Palaeogeogr. Palaeoclimatol. Palaeoecol. 372, 108-122 (2013).

6. R. S. Seymour, Paleobiology 5, 1-11 (1979).

7. N. O. Ratsimbaholison, R. N. Felice, P. M. O'Connor, Acta Palaeontol. Pol. (2016).

8. T. D. Carr, J. Vertebr. Paleontol. 19, 497-520 (1999).

9. D. Codron, C. Carbone, D. W. H. Müller, M. Clauss, Biol. Lett. 8, 620-623 (2012).

10. E. E. Werner, J. F. Gilliam, Annu. Rev. Ecol. Syst. 15, 393-425 (1984).

11. D. Codron, C. Carbone, M. Clauss, PLOS ONE 8, e77110 (2013).

12. H. N. Woodward et al., Sci. Adv. 6, eaax6250 (2020).

13. D. Purwandana et al., Naturwissenschaften 103, 27 (2016).

14. G. M. Erickson, K. C. Rogers, S. A. Yerby, Nature 412, 429-433 (2001).

15. J. R. Horner, A. De Ricqlès, K. Padian, J. Vertebr. Paleontol. 20, 115-129 (2000).

16. G. M. Erickson, P. J. Currie, B. D. Inouye, A. A. Winn, Science 313, 213-217 (2006).

17. G. M. Erickson, P. J. Currie, B. D. Inouye, A. A. Winn, Can. J. Earth Sci. 47, 12691275 (2010).

18. P. Gignac, H. O’Brien, Integr. Comp. Biol. 56, 449-458 (2016).

19. D. J. Varricchio, Hist. Biol. 23, 91-107 (2011).

20. The Paleobiology Database (2020); https://paleobiodb.org/

21. Materials and methods are available as supplementary materials.

22. F. A. Smith et al., Science 330, 1216-1219 (2010).

23. J. B. Foster, Nature 202, 234-235 (1964).

24. D. Codron, J. Codron, M. Sponheimer, M. Clauss, Front. Ecol. Evol. 4, (2016).

25. S. L. Brusatte, M. J. Benton, M. Ruta, G. T. Lloyd, Science 321, 1485-1488 (2008).

26. R. B. J. Benson, G. Hunt, M. T. Carrano, N. Campione, Palaeontology 61, 13-48

(2017).

27. R. B. J. Benson et al., PLOS Biol. 12, e1001853 (2014).

28. K. T. Bates, R. B. J. Benson, P. L. Falkingham, Paleobiology 38, 486-507 (2016).

29. C. Foth, B. P. Hedrick, M. D. Ezcurra, PeerJ 4, e1589 (2016).

30. E. J. Rayfield et al., Nature 409, 1033-1037 (2001).

31. R. Delcourt, Sci. Rep. 8, 9730 (2018).

32. J. O. Farlow, T. R. Holtz Jr.., Paleontol. Soc. Pap. 8, 251-266 (2002).

33. T. D. Carr, PeerJ 8, e9192 (2020).

34. A. R. Fiorillo, R. A. Gangloff, J. Vertebr. Paleontol. 20, 675-682 (2001).

35. M. J. Ryan, P. J. Currie, J. D. Gardner, M. K. Vickaryous, J. M. Lavigne, GAIA 15, $123-$ $133(2000)$. 
36. S. Wang et al., Curr. Biol. 27, 144-148 (2017).

37. G. M. Erickson et al., Nature 430, 772-775 (2004).

38. P. J. Bybee, A. H. Lee, E. T. Lamm, J. Morphol. 267, 347-359 (2006). 


\title{
Supplementary Materials for
}

\section{The influence of juvenile dinosaurs on community structure and diversity}

\author{
Katlin Schroeder*, S. Kathleen Lyons, Felisa A. Smith \\ *Corresponding author. Email: maguire@unm.edu
}

Published 26 February 2021, Science 371, 941 (2021)

DOI: $10.1126 /$ science.abd9220

This PDF file includes:

Materials and Methods

Supplementary Text

Figs. S1 and S2

Tables $\mathrm{S} 1$ to $\mathrm{S} 7$

References

Other Supplementary Material for this manuscript includes the following:

(available at science.sciencemag.org/content/371/6532/941/suppl/DC1)

MDAR Reproducibility Checklist (PDF) 


\section{Materials and Methods}

Data

Dinosaur assemblages were identified by downloading all vertebrate occurrences known to species or genus level between 200Ma and 65MA from the Paleobiology Database (PaleoDB https://paleobiodb.org/\#/ download 6 August, 2018). Using associated depositional environment and taxonomic information, the vertebrate database was limited to only terrestrial organisms, excluding amphibians, pseudosuchians, champsosaurs and ichnotaxa. Taxa present in formations were confirmed against the most recent available literature, as of November, 2020. Synonymous taxa or otherwise duplicated taxa were removed. Taxa that could not be identified to genus level were included as "Taxon X". GPS locality data for all formations between 200MA and 65MA was downloaded from PaleoDB to create a minimally convex polygon for each possible formation.

Any attempt to recreate local assemblages must include all potentially interacting species, while excluding those that would have been separated by either space or time. We argue it is acceptable to substitute formation for home range in the case of non-avian dinosaurs, as range increases with body size. Reaching masses of $80,000 \mathrm{~kg}$, it is not unrealistic for gigantic nonavian dinosaurs to have home ranges that spanned the entirety of a geologic formation, particularly as none of the included formations exceed the modern range of some mammalian megafauna (39). Further, our research is focused on the overall shape of body size diversity distributions, so while there is time-averaging in our dataset, this is also acceptable, as we are examining the presence/absence of body sizes, rather than the exact species identified within the assemblage. Wherever possible, formations were reduced to biostratigraphic groups recognized in the literature to remedy this potential issue, resulting in an average time span of 4.8 million years for our formations. Only formations with at least 5 species were included in the overall dataset, and only formations containing at least 3 carnivores were included in the carnivore test set.

Mass estimates from the literature were added to the final dataset of terrestrial vertebrates. When a range of masses were available for one species, the average was used to normalize for variability within mass estimate techniques, which included, (but were not limited to) volumetric, limb bone circumference, limb bone length, and polynomial regression. Where no mass estimate was available, estimates from similarly sized species within the same genus, family or order were substituted. Species were assigned to a $0.5 \log (10)$ bin based on mass. While it is noted that a wide variety of mass estimate techniques are included within this dataset, as all analyses were performed on $\log 10$ scale, variability between estimates did not have an appreciable effect on our results.

All species were assigned a basic trophic level designator based on species morphological and ecological descriptions in the literature. Species were categorized individually, regardless of taxonomic affinity in order to most accurately capture the intricacies of dinosaur community structuring. "Carnivores" and "Herbivores" were identified as any organism never having been contested in the literature as a non-carnivore or non-herbivore, respectively. As only a small percentage of the species included in our analyses fell outside of these two main categories, we subdivided the "omnivore" group in two, which were then added to either the "carnivore" or "herbivore" group as appropriate. "Meat-dominant omnivore" was assigned to any species that had been suggested as an omnivore after an initial designation of carnivore, or which was identified as an omnivore with identifiable faunivore morphology, and were grouped with carnivores for analysis. A similar method was used to designate "plant-dominant omnivore", 
which were grouped with herbivores. Recent accounts of dietary remnants, such as stomach contents and coprolites, were also considered when grouping species into tropic categories. While we endeavored to be as accurate as possible with these descriptions, some species, particularly enigmatic species within caenagnathidae and oviraptoridae, have yet to be definitively described within a single dietary category. Our inclusion of these species within the "meat-dominant omnivore" group may prove to be inaccurate as more evidence is presented in the literature. However, these enigmatic species represent an exceedingly small portion of the overall sample tested (1.6\%) and therefore are unlikely to significantly change our results when grouped as herbivores rather than carnivores.

\section{$\underline{\text { Statistical Analysis }}$}

To determine the shape of the M-S distribution, each dinosaur assemblage, as well as each carnivore and herbivore assemblage was tested for fit with K-S Goodness of Fit (alpha=0.05) against the global dataset, bootstrapped 1,000 times. Bimodality was assessed with Sarle's Bimodality Coefficient, summary statistics, and fit to quadratic and $4^{\text {th }}$ degree polynomial regressions. Bimodality was further tested through likelihood of bin occupancy for the entire dataset, as well as on carnivores and herbivores separately. To determine the effect of the presence of megatheropods in an assemblage, a Kendal Rank test was performed on 39 carnivore guild distributions. Likelihood of bin occupancy was further tested in relation to occupancy of surrounding mass bins to determine the influence of any larger species on occupancy.

\section{Ontogenetic Biomass Calculations}

In order to identify potential competitive exclusion in dinosaur assemblages, biomass was calculated for megatheropods from nine formations from the total dataset. These formations were selected because they represent multiple geographic locations and geologic periods, and contain theropods for which growth and survivorship curves have been calculated. Growth rates from Erickson and Bybee were multiplied by survivorship of the same species from Erickson, using a 1,000-individual cohort. As it has been hypothesized that giant theropods would all employ a similar rapid growth curve, and as neither growth curves nor survivorship curves for Acrocanthosaurus atokensis and Siats meekerorum were available in the primary literature, growth curves for these species were created by averaging growth rates from Tyrannosaurus rex for its increased size, and Allosaurus fragilis for its taxonomic similarity to A.atokensis and $S$. meekerorum, Survivorship curves from Daspletosaurus torosus were substituted directly, as the mass of all three species is similar. The same method was utilized for Tarbosaurus bataar, averaging curves from $T$. rex and D. torosus. Biomass estimates for Achillobator giganticus were not included as there are no existing comparable growth curves or survivorship estimates available.

The relative biomass for juveniles and adults of each species was multiplied by the proportional biomass for each mass bin, normalized so the adult proportion $=1$. Juveniles were defined as individuals in the cohort that had not reached somatic maturity based on asymptotic growth (approximately age 16). 


\section{Supplementary Text}

Inclusion and exclusion of taxa

Our database of 557 non-avian dinosaur taxa are based on downloads from the Paleobiology Database, an extensive recording of all published taxa, updated and maintained by a group of paleontologists from around the globe. Downloads from the PBDB provided a baseline of all potential taxa to be found in a community, which was then independently verified against the literature, as of November, 2020. Sources for every occurrence of every species were identified independent from the PBDB to confirm their accuracy temporally and spatially, and are listed in Table S1. All obsolete taxa (e.g. Deinodon) were excluded. Morphological and phylogenetic reassessments were followed as much as possible, and taxonomy was updated accordingly, specifically pertaining to the following species:

Amtosaurus archibaldi- fragmentary remains that have been described with ankylosaur or hadrosaur affinities have since been renamed as Bissektipelta (40)

Anatotitan copei- hadrosaur material from western North America which most recently has been demonstrated to be the remains of Edmontosaurus (41).

Chialingosaurus kuani- based solely on juvenile remains from the Shaximiao formation that cannot be distinguished as a unique taxon, and is therefore a nomen dubium (42)

Chungkingosaurus jiangbeiensis- has been identified as a possible juvenile of Tuojiangosaurus (43)

Compsosuchus solus- based on fragmentary remains from the Lameta formation, has since been determined to be a nomen dubium and is therefore listed as "Noasaurid C" in this paper (44)

Drinker nisti- neornithischian remains from this species were reclassified together with at least one other taxon as a single taxon Nanosaurus agilis (45)

145 Iguanodon dawsoni- limited post-cranial material from the Wadhurst Clay formation that has since been reclassified as Barilium dawsoni (46)

Iguanodon orientalis- poorly described skull material from Khuren Dukh that has since been reclassified as Altirhinus kurzanovi (47)

Jubbulpuria tenuis- based on fragmentary remains from the Lameta formation, has been determined to be a nomen dubium and is therefore listed as "Theropod B" in this paper (44)

Koutalisaurus kohlerorum- synonymous with Pararhabdodon (48) 
Majungatholus atopus- originally described as a new pachycephalosaur, material has been reassigned to Majungasaurus (50)

Maleevus disparoserratus- originally described as a new ankylosaur from the Bayan Shireh formation, has since been determined to be a junior synonym of Pinacosaurus (51)

Nanotyrannus- based on a skull originally described as Gorgosaurus lancensis from the Hell 165 Creek formation, Nanotyrannus was erected as a new genus of pygmy tyrannosaur (52). Following the discovery of a second, more complete skeleton, numerous morphological analyses have overwhelmingly shown the two specimens attributed to $N$. lancensis to be juvenile specimens of Tyrannosaurus rex $(8,12,53-56)$

170 Ornithomimus minutus- based on isolated toe bones from the Laramie formation with likely Alvarezsaurid affinity (57), is listed as "Alvarezsauridae" in this paper

Richardoestesia asiatica- although likely a valid species, this tooth taxon cannot be distinguished from $R$. isosceles and has been included within this taxon in this paper (58)

Styracosaurus ovatus- ceratopsian remains from the Two Medicine formation that have since been reclassified as Rubeosaurus (59)

Titanosaurus madagascariensis- based on fragmentary remains from the Maeverano formation, has been identified as synonymous with Laplatasaurus (60)

Triceratops / Torosaurus- although the validity of the genus Torosaurus has been called into question (61-62) as a possible ontogenetic stage of the earlier named Triceratops, we feel this assertion has yet to be definitively proven, particularly in light of more recent morphometric analyses (63-64). As such, we include both genera in our paper.

Ugrunaaluk kuukpikensis- hadrosaurid remains from the Prince Creek formation that have since been reclassified as remains of juvenile Edmontosaurus (65)

190 Wuerhosaurus mongoliensis- informally named stegosaur material from the Dzunbain formation which has since been formally described as Mongolostegus exspectabilis (66)

Willinakaqe salitralensis- originally described as a new saurolophine hadrosaur from the Allen formation(67), W. salitralensis has since been determined to refer to multiple species and is 195 therefore a nomen dubium (68).

Yunnanosaurus robustus- material from the Lufeng formation represents an ontogenetic series, synonymous with $Y$. huangi (69)

In the interest of capturing as much biodiversity as possible, we removed as few "problematic" taxa as possible. Only those that we found to be definitively synonymous, or those that showed a preponderance of evidence towards synonymizing were excluded. 
Fig. S1.

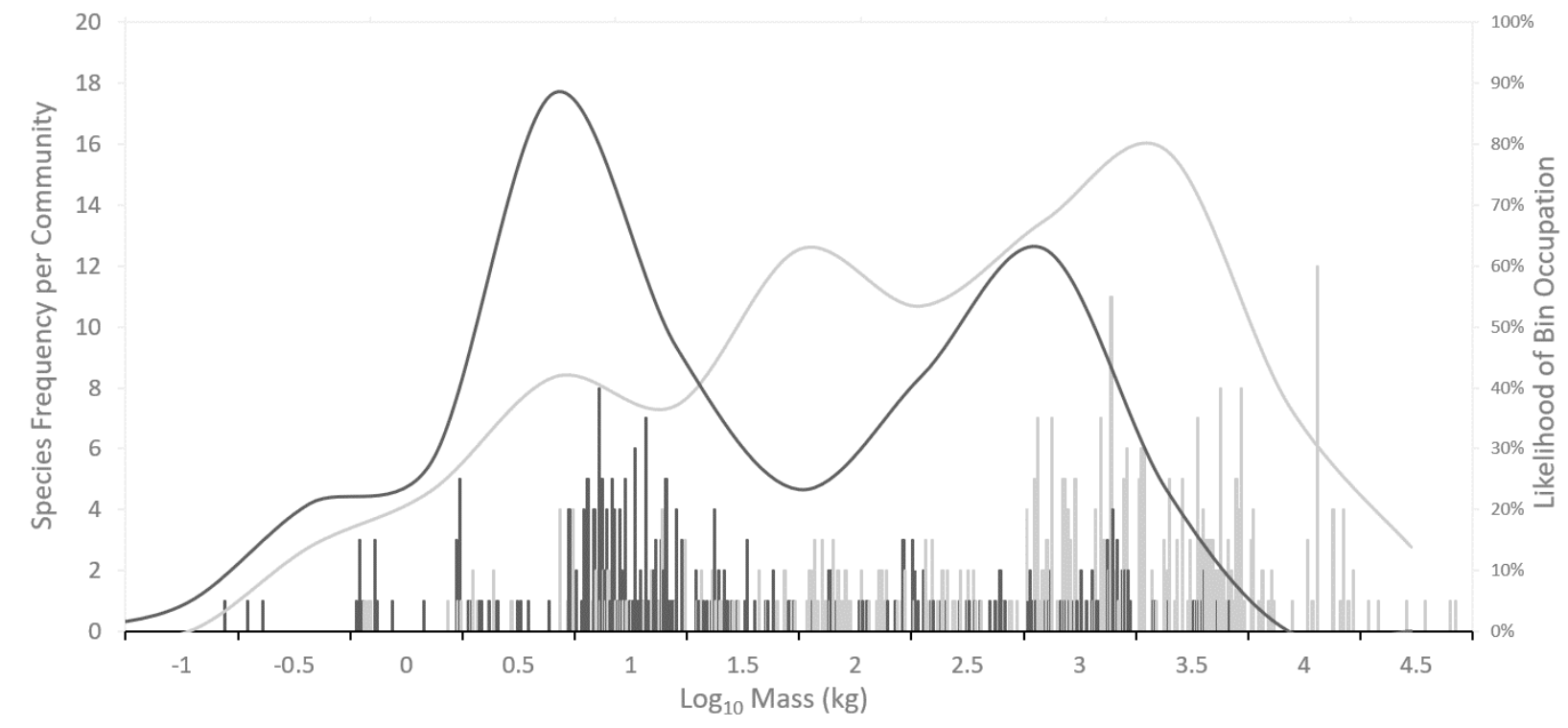

205 Number of species in each mass bin, by guild, in formations from the Jurassic and Cretaceous eras (bars) appears more normal than global distribution. Herbivores (light grey) mirror their leftskewed global distribution, while carnivores exhibit a unique bimodal trend. Likelihood of at least one species existing in mass bin in any formation (lines) shows the distinctly more platykurtic distribution at local scales, as well as the severe drop in likelihood in medium size 210 carnivore bins. 
Fig. S2.

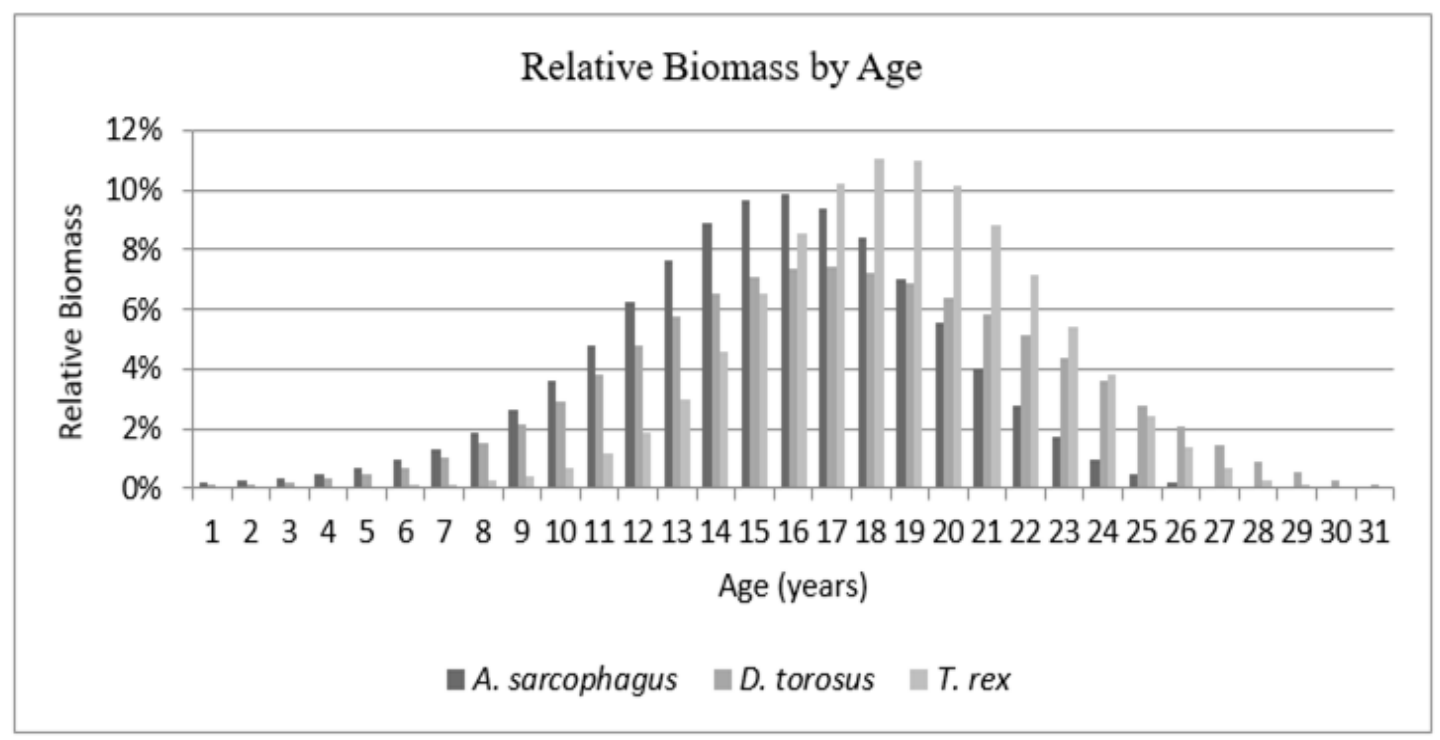

215 Growth vs survivorship of three megatheropods shows biomass is highest at sexual maturity, which occurs shortly before somatic maturity in dinosaurs. 
Table S1.

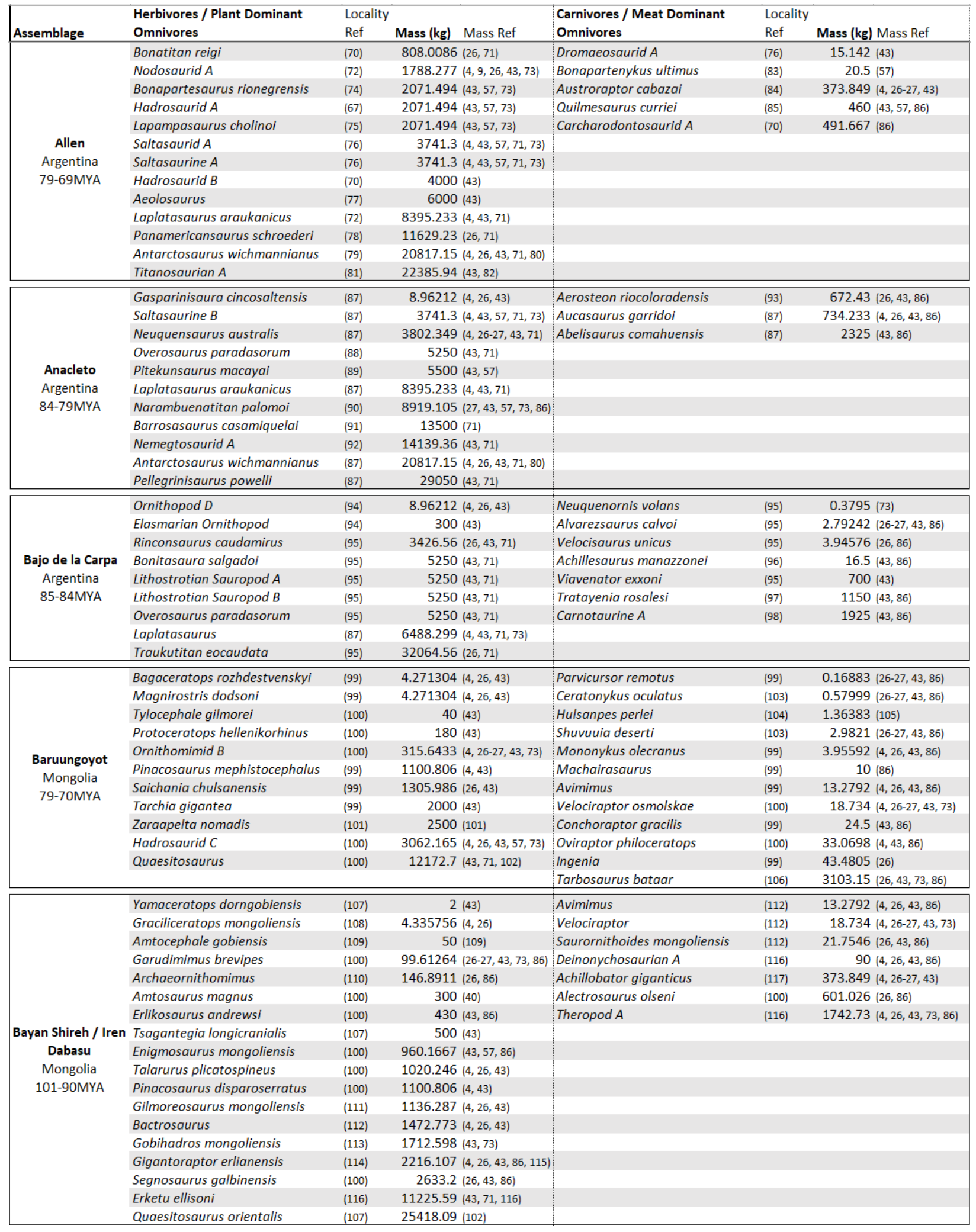




\begin{tabular}{|c|c|c|c|c|c|c|}
\hline \multirow[b]{2}{*}{ Assemblage } & \multirow{2}{*}{$\begin{array}{l}\text { Herbivores / Plant Dominant } \\
\text { Omnivores }\end{array}$} & \multicolumn{2}{|c|}{ Locality } & \multirow{2}{*}{$\begin{array}{l}\text { Carnivores / Meat Dominant } \\
\text { Omnivores }\end{array}$} & \multicolumn{2}{|c|}{ Locality } \\
\hline & & Ref & Mass (kg) Mass Ref & & Ref & Mass (kg) Mass Ref \\
\hline \multirow{7}{*}{$\begin{array}{c}\text { Bissekty } \\
\text { Uzbekistan } \\
\text { 92-90MYA }\end{array}$} & Levnesovia transoxiana & (118) & $175(43)$ & Caenagnathasia martinsoni & (122) & $1.4(43)$ \\
\hline & Bissektipelta archibaldi & $(110)$ & $300(40)$ & Richardoestesia isosceles & (123) & $10.1954(9,86)$ \\
\hline & Ornithomimid C & (119) & $315.6433(4,26-27,43,73)$ & Urbacodon itemirensis & (123) & $10.8333(57,86)$ \\
\hline & Turanoceratops tardabilis & $(120)$ & $483.7592(26,43)$ & Itemirus medullaris & (124) & $12.422(26)$ \\
\hline & Bactrosaurus & (104) & $1472.773(4,26,43)$ & Paronychodon asiaticus & (123) & $12.9875(86)$ \\
\hline & Therizinosauroidea & (120) & $4750(43,86)$ & Timurlengia euotica & (125) & $220(125)$ \\
\hline & Non-lithostrotian Titanosaur A & (121) & $7000(43)$ & & & \\
\hline \multirow{7}{*}{$\begin{array}{c}\text { Callovian Oxford } \\
\text { Clay } \\
\text { United Kingdom } \\
\text { 166-163.5MYA }\end{array}$} & Callovosaurus leedsi & $(126)$ & $108.3666(4,27)$ & Eustreptospondylus oxoniensis & (131) & $348.725(4,26,43,86)$ \\
\hline & Sarcolestes leedsi & (127) & $187.5(57)$ & Metriacanthosaurus parkeri & (132) & $1184.89(26,86)$ \\
\hline & Lexovisaurus durobrivensis & (126) & $2155.233(4,43)$ & & & \\
\hline & Omosaurus durobrivensis & (128) & $2155.233(4,43)$ & & & \\
\hline & Loricatosaurus priscus & (104) & $2257.871(4,26,57,73)$ & & & \\
\hline & Cetiosauriscus stewarti & (129) & $12160.95(4,26,43,71)$ & & & \\
\hline & Cetiosaurus & (130) & $12232.49(4,26,43,71)$ & & & \\
\hline \multirow{10}{*}{$\begin{array}{c}\text { Cedar Mountain } \\
\text { Mussentuchit } \\
\text { United States } \\
\text { 102-95.64MYA }\end{array}$} & Neoceratopsian A & (133) & $1.5(134)$ & Velociraptorine $A$ & (142) & $5.82906(4,43,86)$ \\
\hline & Zephyrosaurus & (135) & $20(43)$ & Richardoestesia & (142) & $10.2398(9,43,86)$ \\
\hline & Pachycephalosaurid A & (136) & $27.6576(4,26,43)$ & Paronychodon & (142) & $12.9875(9,26,43,86)$ \\
\hline & Animantarx ramaljonesi & (137) & $411.8695(4,26,43)$ & Caenagnathoid A & (136) & $13.2792(4,26,43,86)$ \\
\hline & Ornithopod E & (136) & $500(43)$ & Troodontid C & (142) & $21.7546(26,43,86)$ \\
\hline & Tenontosaurus & (138) & $818.362(4,26-27,43)$ & Dromaeosaurine A & (142) & $83.1907(4,26,43,86)$ \\
\hline & Eolambia caroljonesa & (139) & $1000(43)$ & Alectrosaurus & (135) & $601.026(26,86)$ \\
\hline & Peloroplites cedrimontanus & (140) & $2000(43)$ & Siats meekerorum & (143) & $3950(86,143)$ \\
\hline & Cedarpelta bilbeyhallorum & (140) & $5000(43)$ & & & \\
\hline & Astrodon & (135) & $10800(71,141)$ & & & \\
\hline \multirow{8}{*}{$\begin{array}{c}\text { Cloverly } \\
\text { United States } \\
\text { 113-100.5MYA }\end{array}$} & Aquilops americanus & (134) & $1.5(134)$ & Microvenator celer & (144) & $3.62685(4,26)$ \\
\hline & Zephyrosaurus schaffi & (144) & $20(43)$ & Deinonychus antirrhopus & (144) & $83.1907(4,26,43,86)$ \\
\hline & Ornithomimus velox & (104) & $175.6667(9,43,86)$ & Acrocanthosaurus atokensis & (144) & $4558.8(4,26,43,86)$ \\
\hline & Tenontosaurus tilletti & (144) & $818.362(4,26-27,43)$ & & & \\
\hline & Sauropelta edwardsorum & (144) & $2000(43)$ & & & \\
\hline & Tatankacephalus cooneyorum & (144) & $2505.082(43,145)$ & & & \\
\hline & Rugocaudia coonevi & (146) & $6300(71)$ & & & \\
\hline & Sauroposeidon proteles & (144) & $34146.02(26,43,147)$ & & & \\
\hline \multirow{14}{*}{$\begin{array}{c}\text { Dinosaur Park MA } \\
\text { 1b } \\
\text { Canada } \\
\text { 76-75.5MYA }\end{array}$} & Stegoceras validum & (148) & $27.6576(4,26,43)$ & Hesperonychus elizabethae & $(152)$ & $2.55(43,86)$ \\
\hline & Hanssuesia sternbergi & (148) & $28.07162(43)$ & Bambiraptor feinbergi & (153) & $5.82906(4,43,86)$ \\
\hline & Prencephale & (148) & $38.50373(26,43)$ & Richardoestesia isosceles & (153) & $10.1954(9,86)$ \\
\hline & Struthiomimus altus & (149) & $215.0365(4,26-27,43,73)$ & Richardoestesia gilmorei & (153) & $10.3284(9,43,86)$ \\
\hline & Panoplosaurus mirus & (150) & $1436.424(26,43)$ & Zapsalis abradens & (153) & $12.3333(86,104)$ \\
\hline & Euoplocephalus tutus & (150) & $1617.899(4,26,43)$ & Dromaeosaurus albertensis & (153) & $14.7467(9,43,86)$ \\
\hline & Centrosaurus apertus & (150) & $2214.703(4,26,43)$ & Saurornitholestes langstoni & (153) & $23.4053(4,9,26,43,57)$ \\
\hline & Chasmosaurus belli & (150) & $2244.365(4,26,43)$ & Pectinodon & (153) & $47.3759(26)$ \\
\hline & Chasmosaurus russelli & (151) & $2258.128(4,26-27,43)$ & Caenagnathus collinsi & (154) & $61.5(43,155)$ \\
\hline & Edmontonia rugosidens & (150) & $2265.253(26,43)$ & Troodon formosus & (153) & $68.1649(4,26,43,86)$ \\
\hline & Corythosaurus intermedius & (104) & $2977.832(26,43)$ & Chirostenotes pergracilis & (154) & $74.7759(9,43,73,86)$ \\
\hline & Lambeosaurus lambei & (150) & $3248.555(4,26,43)$ & Gorgosaurus libratus & (156) & $2554.78(4,26,43,86)$ \\
\hline & Lambeosaurus clavinitialis & (150) & $3300(43)$ & Daspletosaurus & (156) & $2565.55(4,43,73,86)$ \\
\hline & Parasaurolophus walkeri & (150) & $3549.646(4,26,43)$ & & & \\
\hline \multirow{14}{*}{$\begin{array}{l}\text { Djadokhta } \\
\text { Mongolia } \\
\text { 75-71MYA }\end{array}$} & Bagaceratops & (157) & $4.271304(4,26,43)$ & Parvicursor remotus & (159) & $0.16883(26-27,43,86)$ \\
\hline & Goyocephale lattermorei & $(100)$ & $27.6576(4,26,43)$ & Mahakala omnogovae & $(160)$ & $0.53745(26,43,86)$ \\
\hline & Oviraptor philoceratops & $(100)$ & $33.06984(4,43,86)$ & Halszkaraptor escuilliei & (161) & $0.76234(26,161)$ \\
\hline & Protoceratops andrewsi & $(100)$ & $99.57581(4,26,43)$ & Shuvuuia deserti & (162) & $2.9821(26-27,43,86)$ \\
\hline & Aepyornithomimus tugrikinensis & (158) & $105.9753(158)$ & Gobivenator mongoliensis & (113) & $12.7809(26,43,86)$ \\
\hline & Udanoceratops tschizhovi & (110) & $736.087(26,43)$ & Avimimus portentosus & $(100)$ & $13.2792(4,26,43,86)$ \\
\hline & Pinacosaurus grangeri & $(100)$ & $1100.806(4,43)$ & Byronosaurus & (163) & $13.6(43,86)$ \\
\hline & Nemegtosaurus & $(100)$ & $14139.36(43,71)$ & Velociraptor mongoliensis & $(100)$ & $18.734(4,26-27,43,73)$ \\
\hline & & & & Kol ghuva & (164) & $20(43)$ \\
\hline & & & & Tsaagan mangas & (165) & $20.5(43,86)$ \\
\hline & & & & Saurornithoides mongoliensis & $(100)$ & $21.7546(26,43,86)$ \\
\hline & & & & Khaan mckennai & (166) & $23.9839(26-27)$ \\
\hline & & & & Citipati osmolskae & (116) & $93.9976(26,43,86)$ \\
\hline & & & & Tyrannosauroid & (99) & $3103.15(26,43,73,86)$ \\
\hline
\end{tabular}




\begin{tabular}{|c|c|c|c|c|c|c|}
\hline Assemblage & $\begin{array}{l}\text { Herbivores / Plant Dominant } \\
\text { Omnivores }\end{array}$ & $\begin{array}{l}\text { Locality } \\
\text { Ref }\end{array}$ & Mass (kg) Mass Ref & $\begin{array}{l}\text { Carnivores / Meat Dominant } \\
\text { Omnivores }\end{array}$ & $\begin{array}{l}\text { Locality } \\
\text { Ref }\end{array}$ & Mass (kg) Mass Ref \\
\hline \multirow{10}{*}{$\begin{array}{l}\text { Griman Creek } \\
\text { Australia } \\
99.6-93.5 \mathrm{MYA}\end{array}$} & Iguanodontian A & $(167-168)$ & $6(43)$ & Noasaurid A & (174) & $13.7896(4,26,43)$ \\
\hline & Ornithopod A & (169) & $8.9165(43,57)$ & Noasaurid B & (174) & $26.5(43,86)$ \\
\hline & Ornithopod B & $(169-170)$ & $20(43)$ & Rapator ornitholestoides & (167) & $156.745(4,26,43,57,73)$ \\
\hline & Weewarrasaurus pobeni & (168-169) & $50(169)$ & Megaraptorid A & (175) & $201.729(26,43,86)$ \\
\hline & Ornithopod C & $(169,171)$ & $90(43)$ & Avetheropoda A & (175) & $672.43(26,43,86)$ \\
\hline & Ankylosaurian A & (168) & $300(43)$ & & & \\
\hline & Fostoria dhimbangunmal & $(168,172)$ & $355.8834(27,43)$ & & & \\
\hline & Ankylopollexian A & $(168-169)$ & $910.3948(4,26-27,43)$ & & & \\
\hline & Iguanodontian B & (169) & $4225.792(4,9,27,73)$ & & & \\
\hline & Titanosaurian B & $(168,173)$ & $6000(43)$ & & & \\
\hline \multirow{3}{*}{$\begin{array}{c}\text { Hanson } \\
\text { Antarctica } \\
\text { 194-188MYA }\end{array}$} & Prosauropod A & (104) & $554.9922(4,9,71,73,176)$ & Coelophysoid A & $(178-179)$ & $6(43,178,180)$ \\
\hline & Glacialisaurus hammeri & (177) & $745(71,177)$ & Coelophysoid B & (179) & $25.0459(26,43,86)$ \\
\hline & & & & Cryolophosaurus ellioti & (181) & $349.387(26,43)$ \\
\hline \multirow{12}{*}{$\begin{array}{c}\text { Hell Creek } \\
\text { United States } \\
66.8-66 \mathrm{MYA}\end{array}$} & Orodromeus makelai & (182) & $15.1695(4,26)$ & Richardoestesia isosceles & (196) & $10.1954(9,86)$ \\
\hline & Stegoceras & (183) & $27.6576(4,26,43)$ & Richardoestesia gilmorei & (197) & $10.3284(9,43,86)$ \\
\hline & Citipes elegans & (184) & $56.36128(9,43,185)$ & Acheroraptor temertyorum & (198) & $18.5(86,199)$ \\
\hline & Sphaerotholus buchholtzae & (186) & $68.33955(43,57,73)$ & Saurornitholestes & (197) & $23.7026(4,9,26,43,57)$ \\
\hline & Thescelosaurus & (187) & $170.3897(4,26,43)$ & Pectinodon bakkeri & (200) & $47.3759(26)$ \\
\hline & Leptoceratops gracilis & (188) & $212.9821(4,26,43)$ & Anzu wyliei & (201) & $246.013(43,86,201)$ \\
\hline & Ornithomimus & (189) & $221.4634(4,9,26-27,43)$ & Dakotaraptor steini & (202) & $285(43,86)$ \\
\hline & Pachycephalosaurus wyomingensis & (190) & $342.0206(4,26,43)$ & Tyrannosaurus rex & (55) & $6428.6(4,26,43,73,86)$ \\
\hline & Ankylosaurus magniventris & (191) & $3872.272(4,26,43)$ & & & \\
\hline & Edmontosaurus annectens & (192) & $4595.999(4,26,43)$ & & & \\
\hline & Torosaurus & $(63,193)$ & $7598.517(4,26-27,43,73)$ & & & \\
\hline & Triceratops horridus & $(194)$ & $8318.221(4,26-27,43,73)$ & & & \\
\hline \multirow{9}{*}{$\begin{array}{l}\text { Horseshoe Canyon } \\
\text { Drumheller- } \\
\text { Horsethief } \\
\text { Canada } \\
\text { 73.1-71.5MYA }\end{array}$} & Ornithomimus edmontonicus & (149) & $138.7235(4,26-27,73)$ & Richardoestesia gilmorei & (203) & $10.3284(9,43,86)$ \\
\hline & Struthiomimus & (149) & $215.0365(4,26-27,43,73)$ & Paronychodon & (149) & $12.9875(9,26,43,86)$ \\
\hline & Arrhinoceratops brachyops & (149) & $1300(43)$ & Dromaeosaurus & (149) & $13.56(9,43,86)$ \\
\hline & Edmontonia longiceps & (149) & $1985.43(4,26,43)$ & Atrociraptor marshalli & (203) & $15.25(43,86)$ \\
\hline & Anodontosaurus lambei & (203) & $2164.816(43,204)$ & Troodontid D & (203) & $22.496(86)$ \\
\hline & Anchiceratops ornatus & (149) & $2229.013(26,43)$ & Albertavenator & (203) & $50(205)$ \\
\hline & Pachyrhinosaurus canadensis & (149) & $3139.13(4,26,43)$ & Epichirostenotes & (149) & $98.0518(86)$ \\
\hline & Edmontosaurus regalis & (149) & $5260.076(4,26,43)$ & Apatoraptor & (203) & $115.552(206)$ \\
\hline & & & & Albertosaurus sarcophagus & (149) & $2345.18(4,26,43,86)$ \\
\hline \multirow{11}{*}{$\begin{array}{c}\text { Judith River Coal } \\
\text { Ridge } \\
\text { Canada } \\
76.2-75.2 \mathrm{MYA}\end{array}$} & Pachycephalosaurid B & (207) & $27.6576(4,26,43)$ & Richardoestesia gilmorei & (208) & $10.3284(9,43,86)$ \\
\hline & Hypsilophodontid A & $(207-208)$ & $170.3897(4,26,43)$ & Zapsalis abradens & (208) & $12.3333(86,104)$ \\
\hline & Avaceratops lammersi & (208) & $579.6361(4,43)$ & Paronychodon & (207) & $12.9875(9,26,43,86)$ \\
\hline & Nodosaurid B & (208) & $1436.424(26,43)$ & Dromaeosaurus albertensis & (208) & $14.7467(9,43,86)$ \\
\hline & Mercuriceratops gemini & (208) & $2070.972(43,209)$ & Troodon formosus & (208) & $68.1649(4,26,43,86)$ \\
\hline & Monoclonius crassus & (207) & $2396.318(43,210)$ & Gorgosaurus libratus & (104) & $2554.78(4,26,43,86)$ \\
\hline & Zuul crurivastator & (211) & $2500(211)$ & & & \\
\hline & Lambeosaurus & (207) & $3117.374(4,26,43)$ & & & \\
\hline & Gryposaurus & (208) & $3428.063(4,26,43)$ & & & \\
\hline & Spiclypeus shipporum & (208) & $3500(208)$ & & & \\
\hline & Hadrosaurid D & $(207-208)$ & $4000(43)$ & & & \\
\hline \multirow{9}{*}{$\begin{array}{l}\text { Kaiparowits } \\
\text { United States } \\
\text { 76.1-74MYA }\end{array}$} & Stegoceras validum & (104) & $27.6576(4,26,43)$ & Richardoestesia & (213) & $10.2398(9,43,86)$ \\
\hline & Hypsilophodontid B & (212) & $40.23712(26,43)$ & Paronychodon & (213) & $12.9875(9,26,43,86)$ \\
\hline & Ornithomimus velox & (213) & $175.6667(9,43,86)$ & Dromaeosaurid B & (213) & $13.56(9,43,86)$ \\
\hline & Akainacephalus johnsoni & (214) & $1500(43)$ & Velociraptorine $B$ & (213) & $23.7026(4,9,26,43,57)$ \\
\hline & Nasutoceratops titusi & (215) & $1500(43)$ & Talos sampsoni & (219) & $29(43,219)$ \\
\hline & Kosmoceratops richardsoni & (216) & $1850(43,216)$ & Hagryphus giganteus & (220) & $80(43,86)$ \\
\hline & Utahceratops gettyi & (216) & $2750(43,216)$ & Teratophoneus curriei & (221) & $1532.18(26,43,86)$ \\
\hline & Gryposaurus monumentensis & (217) & $3000(43)$ & & & \\
\hline & Parasaurolophus cyrtocristatus & (218) & $3292.803(4,43)$ & & & \\
\hline \multirow{6}{*}{$\begin{array}{l}\text { Kayenta/Navajo } \\
\text { Sandstone } \\
\text { United States } \\
\text { 199-182MYA }\end{array}$} & Segisaurus & (222) & $6(43,178,180)$ & Kayentavenator elysiae & (227) & $17.75(43,57)$ \\
\hline & Scutellosaurus lawleri & (223) & $14.8132(26,43)$ & Coelophysis kayentakatae & (228) & $30.1113(26,43)$ \\
\hline & Seitaad ruessi & (224) & $85(43,71)$ & Dilophosaurus wetherilli & (229) & $340.609(4,26-27,43,86)$ \\
\hline & Sarahsaurus aurifontanalis & (225) & $163.7259(26-27,43,71)$ & & & \\
\hline & Ammosaurus & (222) & $355.1782(4,26,43,71)$ & & & \\
\hline & Scelidosaurus & (226) & $500(86)$ & & & \\
\hline
\end{tabular}




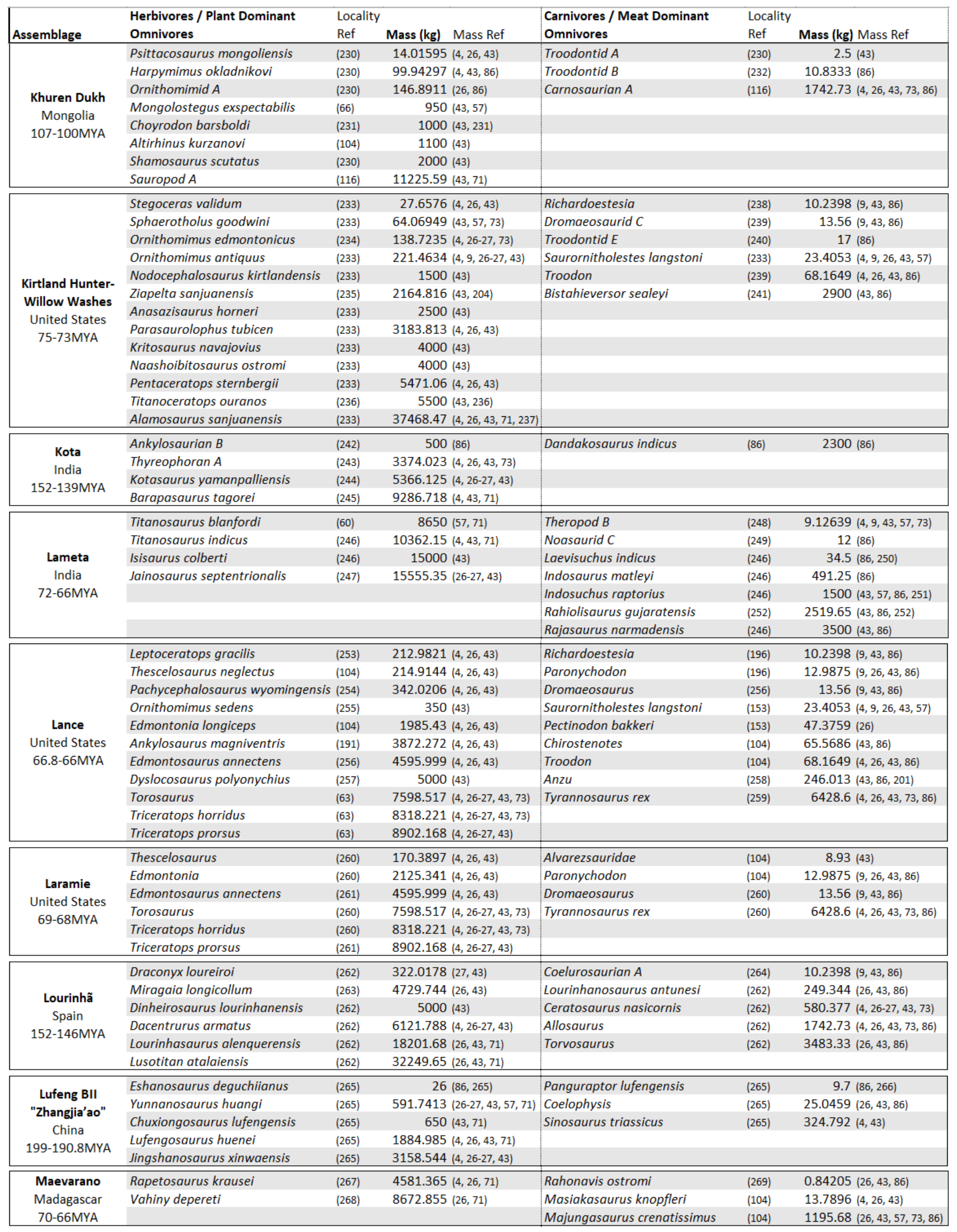




\begin{tabular}{|c|c|c|c|c|c|c|c|}
\hline Assemblage & $\begin{array}{l}\text { Herbivores / Plant Dominant } \\
\text { Omnivores }\end{array}$ & $\begin{array}{l}\text { Locality } \\
\text { Ref }\end{array}$ & Mass (kg) & Mass Ref & $\begin{array}{l}\text { Carnivores / Meat Dominant } \\
\text { Omnivores }\end{array}$ & $\begin{array}{l}\text { Locality } \\
\text { Ref }\end{array}$ & Mass (kg) Mass Ref \\
\hline \multirow{8}{*}{$\begin{array}{c}\text { Milk River } \\
\text { Canada } \\
\text { 84.5-83.5MYA }\end{array}$} & Acrotholus audeti & $(270)$ & & $(270)$ & Richardoestesia isosceles & (272) & $10.1954(9,86)$ \\
\hline & Gryphoceratops morrisoni & (271) & 50 & $(271)$ & Richardoestesia gilmorei & (272) & $10.3284(9,43,86)$ \\
\hline & Brachyceratops & $(104)$ & 1300 & $(43)$ & Zapsalis & (272) & $12.3333(86,104)$ \\
\hline & Paleoscincus & (104) & 1617.899 & $(4,26,43)$ & Atrociraptor & (272) & $15.25(43,86)$ \\
\hline & Nodosaurid C & (272) & 2125.341 & $(4,26,43)$ & Saurornitholestes langstoni & $(104)$ & $23.4053(4,9,26,43,57)$ \\
\hline & Ankylosaurid A & (272) & 2297.224 & $(43)$ & Saurornitholestes & (272) & $23.7026(4,9,26,43,57)$ \\
\hline & Hadrosaurid E & (272) & 3428.063 & $(4,26,43)$ & Tyrannosaurid A & (272) & $2554.78(4,26,43,86)$ \\
\hline & Centrosaurine $A$ & $(104)$ & 3500 & $(43)$ & & & \\
\hline \multirow{18}{*}{$\begin{array}{c}\text { Morrison Brushy } \\
\text { Basin Zone } 3 \\
\text { United States } \\
\text { 150-149.2MYA }\end{array}$} & Nanosaurus agilis & (273) & 24.29303 & $(4,43,57,73)$ & Coelurus fragilis & (273) & $20.3806(26,43,86)$ \\
\hline & Mymoorapelta maysi & (273) & 300 & $(43)$ & Stokesosaurus clevelandi & (273) & $92.5(43,86)$ \\
\hline & Camptosaurus & (273) & 910.3948 & $(4,26-27,43)$ & Marshosaurus bicentesimus & (273) & $212.5(43,86)$ \\
\hline & Stegosaurus stenops & (273) & 3353.66 & $(4,26,43)$ & Ceratosaurus nasicornis & (273) & $580.377(4,26-27,43,73)$ \\
\hline & Ankylosaurus & (273) & 3872.272 & $(4,26,43)$ & Allosaurus fragilis & (273) & $1913.75(4,26,43,73,86)$ \\
\hline & Stegosaurus ungulatus & (273) & 5376.958 & $(26,43)$ & Allosaurus jimmadseni & $(273,278)$ & $1913.75(4,26,43,73,86)$ \\
\hline & Diplodocus carnegii & (273) & 10877.24 & $(9,43,71,274)$ & Torvosaurus & $(273)$ & $3483.33(26,43,86)$ \\
\hline & Camarasaurus lewisi & (273) & 12499.94 & $(4,26,43)$ & Saurophaganax maximus & (273) & $3758.72(26,43,86)$ \\
\hline & Camarasaurus lentus & (273) & 13254.41 & $(4,26,43,71,275)$ & & & \\
\hline & Haplocanthosaurus & (273) & 14217.57 & $(4,26,43,71)$ & & & \\
\hline & Camarasaurus grandis & (273) & 14224.85 & $(26,43,71)$ & & & \\
\hline & Diplodocus longus & (273) & 17338.44 & $(4,26,43,71)$ & & & \\
\hline & Barosaurus & (273) & 21136.82 & $(4,26,43,71)$ & & & \\
\hline & Barosaurus lentus & (273) & 21136.82 & $(4,26,43,71)$ & & & \\
\hline & Camarasaurus supremus & (273) & 22974.7 & $(4,43,71)$ & & & \\
\hline & Apatosaurus louisae & (273) & 25385.81 & $(4,26,43,71)$ & & & \\
\hline & Diplodocus hallorum & $(273,276)$ & 26689.4 & $(26,43)$ & & & \\
\hline & Apatosaurus ajax & $(273)$ & 29708.57 & $(4,26-27,43,71)$ & & & \\
\hline \multirow{13}{*}{$\begin{array}{c}\text { Oldman } \\
\text { Canada } \\
77.5-76.5 \mathrm{MYA}\end{array}$} & Prenocephale brevis & (148) & 10 & (43) & Saurornitholestes langstoni & (286) & $23.4053(4,9,26,43,57)$ \\
\hline & Hanssuesia sternbergi & (148) & 28.07162 & (43) & Daspletosaurus torosus & $(53)$ & $2631.11(4,43,73,86)$ \\
\hline & Struthiomimus altus & (279) & 215.0365 & $(4,26-27,43,73)$ & & & \\
\hline & Wendiceratops pinhornensis & $(280)$ & 1250 & $(43,281)$ & & & \\
\hline & Ankylosaurid B & (282) & 1617.899 & $(4,26,43)$ & & & \\
\hline & Spinops sternbergorum & (283) & 1896.318 & $(43,283)$ & & & \\
\hline & Coronosaurus brinkmani & $(150)$ & 2000 & (43) & & & \\
\hline & Nodosaurid D & $(150)$ & 2125.341 & $(4,26,43)$ & & & \\
\hline & Centrosaurus apertus & (284) & 2214.703 & $(4,26,43)$ & & & \\
\hline & Chasmosaurine A & $(150)$ & 2244.365 & $(4,26,43)$ & & & \\
\hline & Hypacrosaurus stebingeri & (150) & 3293.245 & $(4,43)$ & & & \\
\hline & Albertaceratops nesmoi & (285) & 3500 & (43) & & & \\
\hline & Brachylophosaurus canadensis & $(150)$ & 5729.18 & $(26,43)$ & & & \\
\hline \multirow{8}{*}{$\begin{array}{l}\text { Prince Creek } \\
\text { Alaska } \\
\text { 70.6-69.1MYA }\end{array}$} & Hypsilophodontid C & (287) & 82.79609 & $(4,26-27,43,288)$ & Dromaeosaurus albertensis & (34) & $14.7467(9,43,86)$ \\
\hline & Alaskacephale gangloffi & (287) & 130 & (289) & Saurornitholestes langstoni & (34) & $23.4053(4,9,26,43,57)$ \\
\hline & Ornithomimid D & (287) & 221.4634 & $(4,9,26-27,43)$ & Troodon formosus & (34) & $68.1649(4,26,43,86)$ \\
\hline & Ceratopsid A & $(290)$ & 1300 & (43) & Nanuqsaurus hoglundi & (294) & $750(43,294)$ \\
\hline & Pachyrhinosaurus perotorum & (291) & 2260.778 & $(4,26,43)$ & & & \\
\hline & Pachyrhinosaurus canadensis & (287) & 3139.13 & $(4,26,43)$ & & & \\
\hline & Lambeosaurine $A$ & (292) & 3594.614 & $(4,26,43)$ & & & \\
\hline & Edmontosaurus annectens & $(293)$ & 4595.999 & $(4,26,43)$ & & & \\
\hline \multirow{5}{*}{$\begin{array}{c}\text { Scollard } \\
\text { Canada } \\
66.8-66 \mathrm{MYA}\end{array}$} & Leptoceratops gracilis & (203) & 212.9821 & $(4,26,43)$ & Richardoestesia & (203) & $10.2398(9,43,86)$ \\
\hline & Thescelosaurus neglectus & (203) & 214.9144 & $(4,26,43)$ & Paronychodon & (203) & $12.9875(9,26,43,86)$ \\
\hline & Pachycephalosaurus & (104) & 342.0206 & $(4,26,43)$ & Dromaeosaurus albertensis & (203) & $14.7467(9,43,86)$ \\
\hline & Ankylosaurus magniventris & (203) & 3872.272 & $(4,26,43)$ & Troodon & (203) & $68.1649(4,26,43,86)$ \\
\hline & Triceratops horridus & (203) & 8318.221 & $(4,26-27,43,73)$ & Tyrannosaurus rex & (203) & $6428.6(4,26,43,73,86)$ \\
\hline \multirow{15}{*}{$\begin{array}{c}\text { Shangshaximiao } \\
\text { China } \\
\text { 170.3-166.1MYA }\end{array}$} & Gongbusaurus shiyii & (295) & 20.05596 & $(4,26,43,73,296)$ & Sinocoelurus fragilis & (295) & $25.0459(26,43,86,298)$ \\
\hline & Yandusaurus hongheensis & (295) & 140 & $(43)$ & Chienkosaurus ceratosauroides & (295) & $420(86)$ \\
\hline & Yingshanosaurus jichuanensis & (295) & 1277.148 & $(4,43,73)$ & Szechuanosaurus campi & (295) & $1001.33(57,86,299)$ \\
\hline & Gigantspinosaurus sichuanensis & (295) & 1460.431 & $(26,43)$ & Yangchuanosaurus shangyouensis & $(295)$ & $2950(43,86)$ \\
\hline & Daanosaurus zhangi & (295) & 3517.054 & $(4,43,71,73)$ & Yangchuanosaurus hepingensis & (295) & $3000(43)$ \\
\hline & Tuojiangosaurus multispinus & (295) & 3532.595 & $(4,26-27,43)$ & & & \\
\hline & Omeisaurus maoi & (295) & 6232.125 & $(43,71,73)$ & & & \\
\hline & Mamenchisaurus youngi & (295) & 6746.814 & $(26,43,71)$ & & & \\
\hline & Omeisaurus changshouensis & (295) & 6900 & $(71-71)$ & & & \\
\hline & Mamenchisaurus fuxiensis & (295) & 6967.05 & $(43,57,73,297)$ & & & \\
\hline & Omeisaurus fuxiensis & (295) & 7400 & (71) & & & \\
\hline & Mamenchisaurus constructus & (295) & 11334.89 & $(4,26,43,71)$ & & & \\
\hline & Mamenchisaurus hochuanensis & (295) & 14411.76 & $(4,43,71,73,80)$ & & & \\
\hline & Mamenchisaurus anyueensis & (295) & 25000 & (43) & & & \\
\hline & Mamenchisaurus jingyanensis & $(295)$ & 28500 & $(43,71)$ & & & \\
\hline
\end{tabular}




\begin{tabular}{|c|c|c|c|c|c|c|c|}
\hline \multirow[b]{2}{*}{ Assemblage } & \multirow{2}{*}{$\begin{array}{l}\text { Herbivores / Plant Dominant } \\
\text { Omnivores }\end{array}$} & \multicolumn{3}{|l|}{ Locality } & \multirow{2}{*}{$\begin{array}{l}\text { Carnivores / Meat Dominant } \\
\text { Omnivores }\end{array}$} & \multicolumn{2}{|c|}{ Locality } \\
\hline & & Ref & Mass (kg) & Mass Ref & & Ref & Mass (kg) Mass Ref \\
\hline \multirow{9}{*}{$\begin{array}{c}\text { Tendaguru Middle } \\
\text { Saurian } \\
\text { Tanzania } \\
\text { 157-152MYA }\end{array}$} & Dryosaurus lettowvorbecki & $(300)$ & 80 & (43) & Abelisauroid A & (303) & $10.742(86)$ \\
\hline & Dicraeosaurus hansemanni & (301) & 7268.766 & $(4,9,43,71,274)$ & Non-coelurosaurian Tetanuran A & (303) & $15.1685(4,26,43)$ \\
\hline & Giraffatitan brancai & (302) & 36554.87 & $(4,26-27,43,71)$ & Elaphrosaurus bambergi & (303) & $248.155(4,26,43)$ \\
\hline & & & & & Ceratosaurid B & (303) & $481.333(86)$ \\
\hline & & & & & Ceratosaurid C & $(300)$ & $712.644(86)$ \\
\hline & & & & & Abelisaurid A & (303) & $1002.5(86)$ \\
\hline & & & & & Veterupristisaurus milneri & (303) & $1175(57,86)$ \\
\hline & & & & & Allosaurus tendagurensis & $(300)$ & $2143.4(4,26,43,73,86)$ \\
\hline & & & & & Carcharodontosaurid B & (303) & $2981.55(86)$ \\
\hline \multirow{8}{*}{$\begin{array}{c}\text { Tendaguru Upper } \\
\text { Saurian } \\
\text { Tanzania } \\
\text { 152-145MYA }\end{array}$} & Kentrosaurus aethiopicus & (305) & 1149.464 & $(4,26,43)$ & Abelisauroid A & (303) & $10.742(86)$ \\
\hline & Australodocus bohetti & (305) & 4000 & $(43)$ & Ceratosaurid A & $(300)$ & $481.333(86)$ \\
\hline & Dicraeosaurus sattleri & (301) & 7154.417 & $(26,43,71)$ & Abelisaurid A & (303) & $1002.5(86)$ \\
\hline & Tendaguria tanzaniensis & (305) & 10000 & $(57,71)$ & Ostafrikasaurus crassiserratus & (307) & $1150(86)$ \\
\hline & Tornieria africana & (305) & 12025.71 & $(26-27,43,71)$ & Megalosauroid A & (303) & $1966.67(86)$ \\
\hline & Janenschia robusta & (305) & 12332.74 & $(4,27,43,71)$ & Megalosaurus ingens & (303) & $5700(86,251)$ \\
\hline & Wamweracaudia keranjei & $(306)$ & 12332.74 & $(4,27,43,71)$ & & & \\
\hline & Giraffatitan brancai & $(302)$ & 36554.87 & $(4,26-27,43,71)$ & & & \\
\hline \multirow{7}{*}{$\begin{array}{c}\text { Tremp } \\
\text { Spain } \\
67.6-66 \mathrm{MYA}\end{array}$} & Euhadrosaurian $A$ & (308) & 600 & (43) & Richardoestesia & (311) & $10.2398(9,43,86)$ \\
\hline & Pararhabdodon isonensis & (309) & 1250 & $(43,57)$ & Euronychodon & (311) & $12.9875(86)$ \\
\hline & Nodosaurid E & (104) & 1817.855 & $(4,9,43,73)$ & Paronychodon & (311) & $12.9875(9,26,43,86)$ \\
\hline & Hadrosaurid F & (309) & 3274.599 & $(4,26,43,57,73)$ & Dromaeosaurus & (311) & $13.56(9,43,86)$ \\
\hline & Lambeosaurine $B$ & (309) & 3363.898 & $(4,26,43)$ & Pyroraptor olympius & (311) & $14.7361(86,312)$ \\
\hline & Hypselosaurus & $(310)$ & 6300 & $(57,71)$ & Theropod C & (311) & $757(313)$ \\
\hline & Titanosaurus indicus & $(309)$ & 10362.15 & $(4,43,71)$ & & & \\
\hline \multirow{20}{*}{$\begin{array}{l}\text { Two Medicine } \\
\text { United States } \\
\text { 82.6-74MYA }\end{array}$} & Orodromeus makelai & (314) & 15.1695 & $(4,26)$ & Bambiraptor feinbergi & (324) & $5.82906(4,43,86)$ \\
\hline & Prenoceratops pieganensis & (315) & 20 & (43) & Richardoestesia isosceles & (323) & $10.1954(9,86)$ \\
\hline & Stegoceras validum & (314) & 27.6576 & $(4,26,43)$ & Dromaeosaurus & (314) & $13.56(9,43,86)$ \\
\hline & Hanssuesia sternbergi & (314) & 28.07162 & (43) & Saurornitholestes & (323) & $23.7026(4,9,26,43,57)$ \\
\hline & Montanaceratops & (316) & 134.4206 & $(4,43)$ & Troodon formosus & (323) & $68.1649(4,26,43,86)$ \\
\hline & Cerasinops hodgskissi & (317) & 151.9703 & $(4,26-27,43)$ & Caenagnathus sternbergi & (314) & $74.7759(9,43,73,86,325)$ \\
\hline & Einiosaurus procurvicornis & (318) & 974.7027 & $(4,43)$ & Daspletosaurus horneri & (326) & $2500(43)$ \\
\hline & Brachyceratops montanensis & (318) & 1300 & (43) & Gorgosaurus & (314) & $2554.78(4,26,43,86)$ \\
\hline & Stellasaurus ancellae & (319) & 1521.933 & (319) & & & \\
\hline & Prosaurolophus blackfeetensis & (314) & 1532.758 & (4) & & & \\
\hline & Euoplocephalus tutus & (314) & 1617.899 & $(4,26,43)$ & & & \\
\hline & Achelousaurus horneri & (318) & 1709.393 & $(4,26,43)$ & & & \\
\hline & Oohkotokia horneri & $(320)$ & 1750 & $(43,320)$ & & & \\
\hline & Chasmosaurus & (314) & 2251.247 & $(4,26-27,43)$ & & & \\
\hline & Edmontonia rugosidens & (314) & 2265.253 & $(26,43)$ & & & \\
\hline & Rubeosaurus ovatus & (314) & 2289.302 & $(43,57,321)$ & & & \\
\hline & Maiasaura peeblesorum & (314) & 2873.436 & $(4,26,43)$ & & & \\
\hline & Acristavus gagslarsoni & (322) & 3000 & (43) & & & \\
\hline & Hypacrosaurus stebingeri & (323) & 3293.245 & $(4,43)$ & & & \\
\hline & Gryposaurus latidens & $(323)$ & 3365.19 & $(26,43)$ & & & \\
\hline \multirow{12}{*}{$\begin{array}{c}\text { Upper Elliot } \\
\text { Lesotho } \\
\text { 201-199.3MYA }\end{array}$} & Abrictosaurus consors & (327) & 1.797141 & $(4,26-27,43)$ & Coelophysis rhodesiensis & (336) & $13.0264(26,43)$ \\
\hline & Lycorhinus angustidens & (327) & 3.763795 & $(43,328)$ & Dracovenator regenti & (337) & $217.5(43,86)$ \\
\hline & Pegomastax africanus & (329) & & (329) & & & \\
\hline & Heterodontosaurus tucki & (327) & 4.02759 & $(4,26,43)$ & & & \\
\hline & Lesothosaurus diagnosticus & (327) & 4.959149 & $(4,26,43)$ & & & \\
\hline & Stormbergia dangershoeki & (327) & 35.54129 & $(4,26-27)$ & & & \\
\hline & Arcusaurus pereirabdalorum & (330) & 51.32899 & $(331)$ & & & \\
\hline & Massospondylus kaalae & (332) & 280 & $(43,71)$ & & & \\
\hline & Massospondylus carinatus & (330) & 430.3565 & $(4,26,43,71)$ & & & \\
\hline & Melanorosaurus & (333) & 1300 & (43) & & & \\
\hline & Aardonyx celestae & $(330)$ & 2604.76 & $(4,71,334)$ & & & \\
\hline & Pulanesaura eocollum & $(330)$ & 3200 & $(43,71,335)$ & & & \\
\hline
\end{tabular}




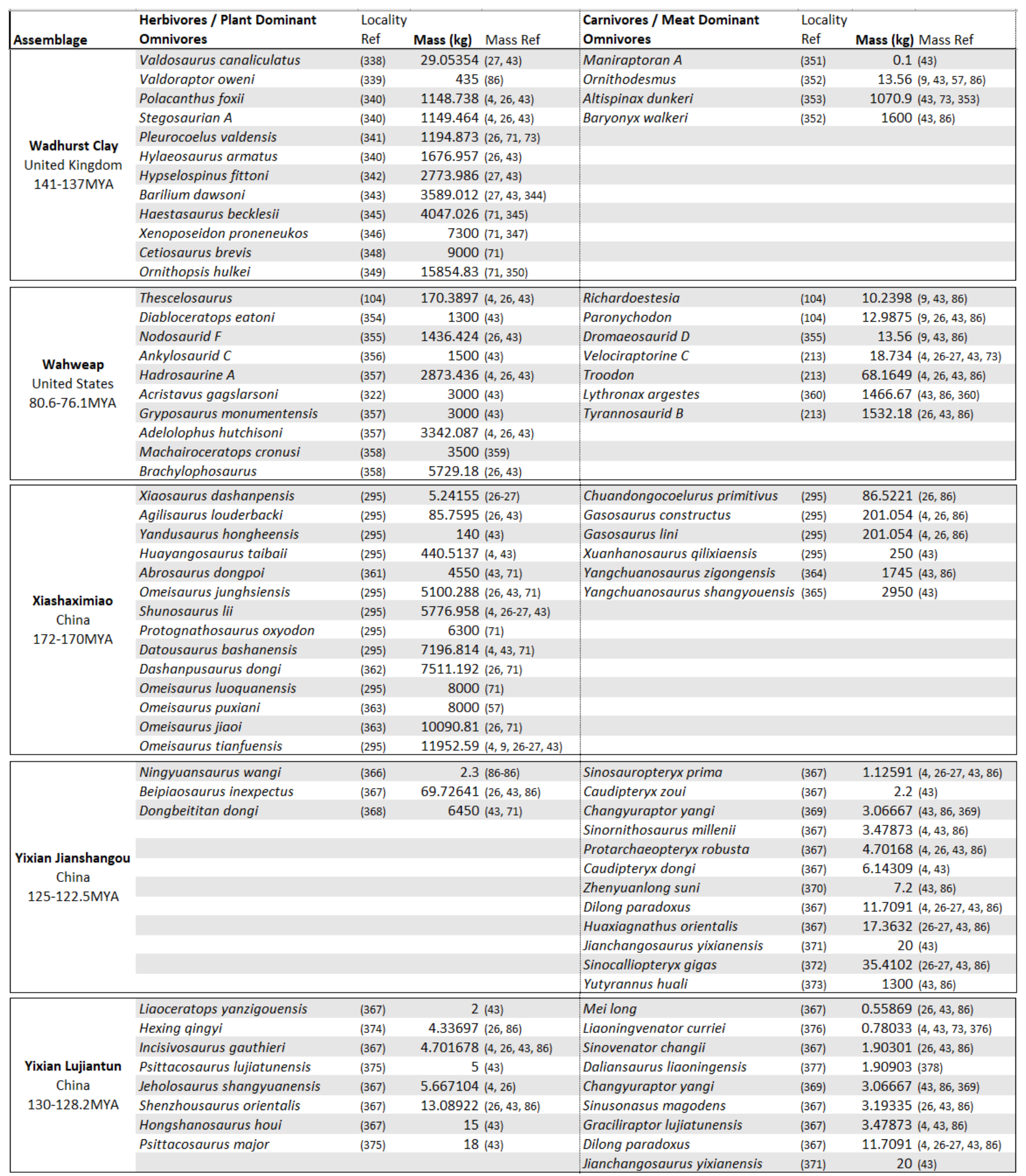

Taxon list for each community with mass in $\mathrm{kg}$ and references 


\section{Table S2.}

\begin{tabular}{|c|c|c|c|c|c|c|c|c|c|c|c|c|c|c|c|c|c|c|c|c|c|c|c|c|}
\hline \multirow[t]{2}{*}{ Formation } & \multirow[b]{2}{*}{$\mathrm{N}$} & \multicolumn{8}{|c|}{ Raw Data $=\log 2($ mass $(\mathrm{kg}))$} & \multicolumn{4}{|c|}{ M-S Distribution } & \multicolumn{2}{|c|}{$\begin{array}{l}\text { KS Goodness of } \\
\text { Fit (vs Global) }\end{array}$} & \multicolumn{3}{|c|}{$\begin{array}{l}\text { Quadratic Regression } \\
\text { (bins) }\end{array}$} & \multicolumn{3}{|c|}{\begin{tabular}{|c} 
4th degree polynomial \\
regression
\end{tabular}} & \multicolumn{3}{|c|}{ Bimodality Coefficient } \\
\hline & & Min & Max & IQR & Q1 & Q3 & Median & Mean & $P_{\text {Mean }}$ & Skew & $P_{\text {skew }}$ & kurtosis & $P_{\text {twot }}$ & & . & & & R2 & & $P$ & & b1 & b2 & $b$ (Sarle) \\
\hline Upper Elliot & 14 & 1.8 & 3200.0 & 643.7 & 4.0 & 647.8 & 43.4 & 582.2 & 0.032 & 0.3142 & 0.157 & -1.201 & 0.555 & 0.4535 & 0.0090 & 3.0044 & 0.1064 & 0.4289 & 1.8307 & 0.2420 & 0.5496 & 0.1159 & 1.7870 & 0.1983 \\
\hline Lufeng BII "Zhangjia'ao" & 8 & 9.7 & 3158.5 & 1551.0 & 25.3 & 1576.2 & 458.3 & 833.9 & 0.254 & -0.5714 & 0.994 & -1.729 & 0.335 & 0.5249 & 0.0020 & 1.3960 & 0.3020 & 0.2587 & 0.5478 & 0.7084 & 0.2675 & 0.4444 & 1.5102 & 20.2253 \\
\hline Kayenta/Navajo Sandstone & 9 & 6.0 & 500.0 & 331.6 & 16.3 & 347.9 & 85.0 & 168.1 & 0.040 & 0.0363 & 0.320 & -1.818 & 0.316 & 0.6402 & 0.0000 & 2.5982 & 0.1351 & 0.3938 & 1.5300 & 0.3051 & 0.5050 & 0.0017 & 1.4454 & 40.1665 \\
\hline Hanson & 5 & 6.0 & 745.0 & 634.5 & 15.5 & 650.0 & 349.4 & 336.1 & 0.132 & -0.7560 & 0.794 & -2.479 & 0.171 & 5402 & 0000 & 0.7537 & 0.5013 & 0.1585 & 0.3046 & 0.8651 & 0.1688 & 1.0160 & 1.3802 & \\
\hline ashaximiao & 21 & 5.2 & 11952.6 & 7183.5 & 170.5 & 7354.0 & 2950.0 & 3844.0 & 0.608 & -0.6533 & 0.919 & -0.453 & 0.926 & 2532 & 262 & 1.5944 & 0.2613 & 0.2850 & 1.3187 & 0.3622 & 0.467 & 0.4728 & 2.3752 & 20.2503 \\
\hline langshaximiao & 1 & 20.1 & 28500.0 & 6044.3 & 1139.2 & 7183.5 & 3532.6 & 6398.5 & 0.188 & -1.4961 & 0.146 & 1.883 & 0.129 & 1693 & 7670 & 1.8521 & 0.2183 & 0.3165 & 6.5876 & 0.0220 & 0.8145 & 2.4802 & 4.1911 & 10.4520 \\
\hline allovian Oxford Clay & 0 & 108.4 & 12232.5 & 4565.9 & 167.7 & 4733.6 & 1670.1 & 3290.0 & 0.472 & 0.3895 & 0.134 & -0.782 & 0.8 & 3249 & 0.128 & 1.4842 & 0.2830 & 0.2706 & 1.1403 & 0.4209 & 0.4319 & 0.1920 & 2.0123 & 30.1877 \\
\hline urian & 12 & 10.7 & 554.9 & 2650.0 & 122.0 & 2772.0 & 741.9 & 4370.2 & & & 0.335 & -0.113 & & & & 18 & 0. & 0.3752 & 33 & 0.3890 & 0.4512 & & & \\
\hline endas & 14 & 10.7 & 36554.9 & 10989.7 & 1112.7 & 12102.5 & 4850.0 & 7561.5 & 0.087 & -1.4106 & 0.186 & 3.114 & 0.053 & 170 & 0.4850 & 3.6299 & 0.0755 & 0.4757 & 7.9123 & 0.0143 & 0.8406 & 2.3352 & 4.7080 & 0.3901 \\
\hline Kota & & 500.0 & 9286.7 & 5926.4 & 1400.0 & 7326.4 & 3374.0 & 4165.4 & 0.152 & -1.2578 & 0.277 & 0.313 & 0.449 & & 1280 & 0.9583 & 0.4236 & 0.1933 & 524 & 0.2196 & 0.5668 & 2.8125 & 2.0781 & \\
\hline Morrison Brushy B & $27 \mid$ & 20.4 & 29708.6 & 16428.0 & 910.4 & 17338.4 & 3872.3 & 9554.0 & 0.132 & -1.1571 & 0.346 & 0.228 & 0.495 & 1476 & 0.7670 & 1.8688 & 0.2158 & 0.3184 & 2.2611 & 0.1778 & 0.6012 & 1.4481 & 2.9734 & 0.3853 \\
\hline $\begin{array}{l}\text { Lourinhã } \\
\text { Crete }\end{array}$ & 11 & 2 & 32249.7 & 99.8 & 2.0 & 21.8 & 3.3 & 8.3 & 254 & 265 & 0.824 & 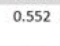 & 0. & 1667 & 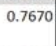 & 11 & 0. & 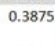 & 1.9438 & 6 & 0.5644 & 0.6516 & 12 & 2 \\
\hline Wadhurst Clay & 17 & 1 & .8 & 3065.1 & 753.0 & 3818.0 & 1600.0 & 3204.3 & 557 & 67 & 0.040 & 73 & 0. & 2896 & 2 & 194 & 0.3641 & 0.2232 & 168 & 11 & ( & 6736 & 93 & 30.6057 \\
\hline Yixian Lujiantun & 17 & 0.6 & 20.0 & 10.4 & 2.0 & 12.4 & 4.3 & 6.7 & 0.000 & 0.1170 & 0.264 & -1.516 & 0.407 & 365 & 0 & 2.1308 & 0.1812 & 0.3476 & 14.2267 & 0.0032 & 0.9046 & 0.0156 & 1.5610 & 0.1946 \\
\hline ian & & 1. & 450.0 & & & 35.4 & 7.2 & 529.0 & & & 0.002 & 1.636 & & & & & 0.1760 & 0.3 & & 0.0 & $c_{-}$ & 2 & & \\
\hline overly & 11 & 1.5 & 34146.0 & 538.8 & 20.0 & 4558.8 & 818.4 & 4601.1 & 0.608 & -0.1915 & 0.535 & -0.889 & 0.7 & 76 & 0.2620 & 1.7993 & 0.2263 & 0.3103 & 358 & 0.5491 & 0.35 & 0.0453 & 1.9665 & 104 \\
\hline uren Du & & 5 & 11225.6 & $=$ & & 2.0 & 975.0 & 6.0 & 0 & \begin{tabular}{|l|l|}
-0.5796 \\
\end{tabular} & 0.997 & -0.3 & & & 100 & & 0.4032 & 0.2 & & 0.7 & $26 \mathrm{c}$ & & & \\
\hline dar $N$ & 18 & .5 & 10800.0 & 236.8 & 2 & 250.0 & 247.5 & 1404.3 & 0.204 & 0.1482 & 0.239 & -1.251 & 0.5 & 224 & 1280 & 570 & 0.3352 & 0.2391 & 52 & 0.6917 & 0.2772 & 248 & 1.7546 & 909 \\
\hline & 2 & 0 & 254 & 7 & 2.0 & 1592.7 & 0.0 & 2131.6 & 0 & -0. & 0.828 & -0.3 & 0.8 & 10 & 00 & & 0.1207 & & & 0.3 & 77 & 39 & & 40 \\
\hline Griman Cre & 15 & 6.0 & 6000.0 & 4 & .0 & 672.4 & 156.7 & 869.2 & 52 & 391 & 0.146 & -0.654 & 0. & 68 & 0 & 00 & 0. & 0.6036 & 97 & 0.1131 & 6644 & 34 & 94 & 08 \\
\hline Bissekty & 14 & 1.4 & 7000.0 & 71 & 0 & 731.0 & 197.5 & 1055.6 & 0 & 35 & 0.210 & -0.676 & 0.8 & 57 & 0 & 19 & 0.2984 & 0.2609 & 93 & 0.6953 & 51 & 186 & 2.1 & 53 \\
\hline jod & 15 & 2.8 & 32064.6 & 5 & 5.5 & 250.0 & 1925.0 & 2.4 & 67 & 69 & 0.834 & -0.828 & 0. & & 20 & 595 & 0.6415 & 0.1050 & 24 & 0.5177 & 0.3756 & 960 & 83 & 743 \\
\hline $\mid \mathbf{k} \mathbf{R} i$ & 17 & 10.2 & 4000.0 & 2406.7 & 19.3 & 2426.0 & 50.0 & 1237.2 & 45 & 20 & 0.237 & -2.035 & 0. & 85 & 0.1280 & 039 & 0.4807 & 0.1673 & 544 & 0.8261 & 0.1955 & 0.0263 & 1.1828 & 2120 \\
\hline acl & & .0 & 29 & 11732.5 & 1927.3 & 13659.8 & 75.0 & 5.8 & 0 & 92 & 0.022 & 5. & 0 & & & & 0 & & & 56 & .780 & .5734 & 54 & 4 \\
\hline Two Medicin & & 5.8 & 3365.2 & 2255.5 & 27.8 & 2283.3 & 1411.0 & 1263.3 & 62 & 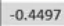 & 0.854 & -1.528 & 0. & 0.4535 & 0.0090 & 1.1325 & 0.3689 & 0.2 & 57 & 0.7284 & 25 & 0.2181 & 1.5248 & B 0.2491 \\
\hline & & & & & & 3.5 & .3 & 3.0 & & -0. & 0.756 & -1.135 & 0. & & & & 0. & 0.2 & & 0.5994 & 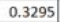 & 40 & 07 & \\
\hline len & 18 & 1 & 22385.9 & 115.1 & 3.8 & 98.8 & 071.5 & 5049.0 & 0 & -1. & 0.370 & 1.116 & 0. & 818 & 0 & 972 & 0.1529 & 0.3747 & 289 & 0.0259 & 8034 & 514 & 3.5131 & 10.3440 \\
\hline & & & & & & & 2000.0 & 1 & & & 0.327 & -0. & & & & & 0. & & & 0. & & & & \\
\hline dith Riv & 17 & \begin{tabular}{|l|}
10.3 \\
\end{tabular} & 4 & 9 & & 6.1 & 1436.4 & 1523.5 & 0 & 87 & 0.745 & -1.696 & 0. & 485 & 0.1280 & 325 & 0.3028 & c & 84 & 259 & ( & 547 & 299 & 270 \\
\hline 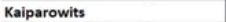 & & 10 & & & & & & & & & & -1.965 & & & & & & & & & & & 43 & \\
\hline Dinos & 27. & 2.6 & 3549.6 & 2241.8 & 23.4 & 5.3 & 1.8 & 1143.5 & 0 & 15 & 0.390 & -1.495 & 0. & 138 & 0.0410 & 834 & 0.3048 & 0.2 & 885 & 0.7019 & 271 & 0029 & 1.5534 & 2033 \\
\hline deth & & 2 & & & 85 & & & & & & & & & & & & & & & & & 274 & 77 & \\
\hline & 19 & .2 & 37 & & 7 & 0.0 & 00.0 & 5.9 & 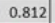 & 008 & 0.351 & -1. & 0.44 & & & 698 & 3319 & 0.2410 & 5260 & ( & 259 & 0000 & 2 & 23 \\
\hline $\mathrm{Hc}$ & & 10 & & & & & 7 & & & & & & & & & & & & & & & & 91 & \\
\hline & & 2 & 2.7 & & 3 & & 1 & .9 & & 0 & 0 & -1. & & & 009 & 630 & 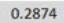 & .2678 & 1 & 8 & 325 & 0670 & D & 85 \\
\hline & 1 & 9.1 & 15 & 1 & & 10 & 2519.6 & & & & 0.718 & -0. & & & & & & & & 50 & ( & & 25 & \\
\hline ince & & 14.7 & .0 & & 8 & 2919.5 & 485.7 & 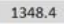 & 0.6 & -0.1062 & 0 & -1.484 & 0. & 82 & 128 & 497 & 0.0261 & 0.5980 & 375 & 0.1153 & 0.662 & 137 & 6044 & 1798 \\
\hline & & 0. & & & & & & & & & & & & & & & & & & & & & & \\
\hline & & 8 & & & & & 3360.7 & & & & 63 & . & & & & & & & & 81 & 07 & 5166 & 44 & 2724 \\
\hline & & .2 & & & & & & & & & & & & & & & & & & 231 & & 1 & 1.5737 & \\
\hline & & & & & & & & & & & 494 & & & & & & & & 03 & 72 & 238 & 68 & 803 & 935 \\
\hline & & 102 & & & & & 2460 & 2 & & & 327 & & & & & & 2940 & & 5949 & 108 & & 9 & 3593 & 2078 \\
\hline & 10 & 10.2 & 8.2 & & 14.3 & & 3.9 & & & & 0.160 & & & & & 37. & & & & & & & 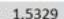 & \\
\hline
\end{tabular}

Summary Statistics, KS Test and polynomial tests for full communities. $\alpha=0.05$, Sarle's $b=$ .555 


\section{Table S3.}

\begin{tabular}{|c|c|c|c|c|c|c|c|c|c|c|c|c|c|c|c|c|c|c|c|c|c|c|c|c|}
\hline \multirow{2}{*}{$\frac{\text { Formation }}{\text { Jurassic }}$} & \multirow[b]{2}{*}{$\mathrm{N}$} & \multicolumn{8}{|c|}{ Raw Data $=$ Log2(mass $(\mathrm{kg}))$} & \multicolumn{4}{|c|}{ M-S Distribution } & \multicolumn{2}{|c|}{$\begin{array}{l}\text { KS Goodness of } \\
\text { Fit (vs Global) }\end{array}$} & \multicolumn{3}{|c|}{$\begin{array}{l}\text { Quadratic Regression } \\
\text { (bins) }\end{array}$} & \multicolumn{3}{|c|}{$\begin{array}{l}\text { 4th degree polynomial } \\
\text { regression }\end{array}$} & \multicolumn{3}{|c|}{ Bimodality Coefficient } \\
\hline & & Min & Max & IQR & Q1 & $\mathrm{Q}^{3}$ & Median & Mean & $P_{\text {Mean }}$ & Skew & $P_{\text {staw }}$ & kurtosis & $P_{\text {turn }}$ & D & $P$ & $\mathbf{F}$ & $\mathrm{P}$ & R2 & $\mathbf{F}$ & $P$ & R2 & b1 & b2 & $\mathrm{b}$ (Sarle) \\
\hline Upper Elliot & 12 & 1.8 & 3200.0 & 1078.6 & 4.0 & 1082.6 & 43.4 & 660.0 & 0.006 & 0.2829 & 0.110 & -1.4683 & 0.391 & 0.7917 & 0.0 & 1.2707 & 0.3317 & 0.2411 & 0.8434 & 0.5454 & 0.3599 & 0.0968 & 1.6143 & 0.1942 \\
\hline Lufeng BII "Zhangjia'ao" & 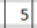 & 26.0 & 3158.5 & 2212.9 & 308.9 & 2521.8 & 650.0 & 1262.3 & 0.262 & -1.7356 & 0.246 & 3.2510 & 0.176 & 0.6667 & 0.0000 & 1.3061 & 0.3229 & 0.2462 & 0.8051 & 0.5643 & 0.3493 & 5.3551 & 2.8128 & 0.5877 \\
\hline yenta/Navajo Sandstone & & 6.0 & 500.0 & 378.8 & 12.6 & 391.4 & 124.4 & 187.5 & 0.007 & -0.3827 & 0.441 & -1.4813 & 0.387 & 0.7273 & 0.0000 & 4.1315 & 0.0586 & 0.5081 & 7250 & 0.1313 & 0.6450 & 0.2289 & 1.6350 & 0.1559 \\
\hline ashaximiao & 15 & 5.2 & 11952.6 & 7860.0 & 140.0 & 8000.0 & 5777.0 & 5019.3 & 0.957 & -1.2712 & 0.616 & 0.8317 & 0.695 & 0.2051 & 0.8430 & 0.7981 & 0.4830 & 0.1663 & .0851 & 0.4414 & 0.4197 & 1.8740 & 3.2042 & 0.4121 \\
\hline miao & 16 & 20.1 & 28500.0 & 8376.6 & 1974.6 & 10351.2 & 489.5 & 7935.8 & 0.452 & -2.0360 & 0.132 & 4.4682 & 0.082 & 0.5000 & 0.0510 & 1.3932 & 0.3026 & 0.2583 & 6460 & 0.0774 & 0.7085 & 4.7586 & 5.8361 & 0.6033 \\
\hline Callovian Oxford Clay & o & 108.4 & 12232.5 & 59557.0 & 128.1 & 9685.2 & 2155.2 & 3920.8 & 0.859 & 0.2765 & 0.112 & -1.3917 & 0.418 & 0.5278 & 0.0510 & 1.0217 & 0.4026 & 0.2035 & 0.6426 & 0.6519 & 0.2999 & 0.1041 & 1.6706 & 0.1680 \\
\hline Tendaguru Middle Saurian & 3 & 80.0 & 36554.9 & 36474.9 & 80.0 & 36554.9 & 7268.8 & 14634.5 & 0.684 & -0.9352 & 0.967 & & & 0.3333 & 0.1700 & 1.1120 & 0.3749 & 0.2175 & 0.6273 & 0.6607 & 0700 & 3.4985 & & \\
\hline Tendaguru Upper Saurian & 8 & 1149.5 & 36554.9 & 7544.1 & 4788.6 & 12332.7 & 11012.9 & 11943.7 & 0.076 & -0.4877 & 0.539 & 0.4208 & 0.882 & 0.3750 & 0.1700 & 4.7277 & 0.0441 & 0.5417 & 6.1118 & 0.0261 & 0.8029 & 0.3238 & 2.5337 & 0.1781 \\
\hline ota & 4 & 500.0 & 9286.7 & 7088.1 & 1218.5 & 8306.6 & $5 \quad 4370.1$ & 4631.7 & 0.528 & -2.0000 & 0.145 & 4.0000 & 0.111 & 0.6389 & 0.0090 & 0.5768 & 0.5834 & 0.1260 & 0.9225 & 0.5086 & 0.3808 & 9.0000 & 2.3333 & 0.6316 \\
\hline Morriso & 19 & 24.3 & 29708.6 & 517783.2 & 353.7 & 21136.8 & 3 13254.4 & 12946.5 & 0.213 & -2.0478 & 0.131 & 3.8594 & 0.118 & 0.6316 & 0.0090 & 1.4041 & 0.3002 & 0.2598 & 557 & 0.3238 & 0.4925 & 4.7014 & 5.6160 & 0.6204 \\
\hline Lourinhã & 6 & 322.0 & 32249.7 & 18085.9 & 627.8 & 21713.7 & 5560.9 & 11 & 0.171 & -0.4401 & 0.492 & 1.3350 & 0.530 & 0.3333 & 0.1700 & 2.5238 & 0.1413 & 0.3869 & .8270 & 0.2426 & 0.5491 & 0.3026 & 2.6006 & 0.1472 \\
\hline \multicolumn{25}{|l|}{ Cretaceous } \\
\hline adh & 13 & 9.1 & 15854.8 & 1524.4 & 49.1 & 673.5 & 174.0 & 883.7 & 0.776 & -2.0 & 0.144 & $5.5 \mathrm{c}$ & 0.040 & & 1.0 & & & & & & & 051 & & 0.5743 \\
\hline ixian & . & 2.0 & 18.0 & 10.1 & 4.4 & 14.5 & 5.3 & 8.5 & 0.000 & -0.4041 & 0.456 & -0.2286 & 0.826 & 0.9333 & 0.0000 & 1.2138 & 0.3464 & 0. & 4.0217 & 0.0639 & 0.7283 & 0.2222 & 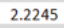 & 0.1716 \\
\hline ixian & 3 & 2.3 & 6450.0 & 6447.7 & 2.3 & 6450.0 & 69.7 & 2174.0 & 0.007 & 0.4233 & 0.078 & & & 0.6 & 0.0000 & 0.1035 & 0.9029 & & 06 & 0.9 & 0.1282 & 0.7166 & & \\
\hline Clover & 8 & 1.5 & 34146.0 & 5292.4 & 58.9 & 5351.3 & 409.2 & & 0.262 & -0.4 & & 0. & 0. & & 0.8430 & & & & & & 74 & 0.3061 & & \\
\hline Chure & 9 & 14.0 & 11225.6 & 5.6 & 123.4 & .0 & 1000.0 & 1959.6 & 0.377 & -0.3645 & 0.427 & -0.1324 & 0.867 & 11 & 0.8430 & 1.6 & 0.2432 & 78 & 44 & 0 & 0.3962 & 35 & 5 & 0. \\
\hline it & 10 & & & & & & & & 0.171 & & & & & & & & & & & & & & & \\
\hline yan & 18 & .0 & 25418.1 & 3.4 & 35.1 & 338.5 & 0.2 & 1.6 & 0.262 & -0.8488 & 0.908 & 0.7106 & 0.756 & 4.0000 & 0.0000 & 1.5294 & 0.2739 & 0. & .0649 & 0. & 0.4152 & 0.8134 & 3.2122 & 0.2657 \\
\hline ima & 10 & 6.0 & & & & & & & 0.03 & & & & 0.505 & & & 2 & & & & & & & & \\
\hline issek & 7 & 175.0 & 7000.0 & 0.0 & 0.0 & .0 & 483.8 & 1.0 & 0.528 & & 0.1 & -2.0 & 0.273 & 34 & & 1.8306 & 0.2215 & 0 & 75 & 0 & 0 & 0.1852 & 94 & 0. \\
\hline ins & 9 & 9.0 & 32064.6 & 9 & 3 & .1 & 152 & & 0.7 & & & & 0.200 & & & & & & & & & & & 0 \\
\hline & 9 & 40.0 & & ) 2789.0 & & & & & 0.776 & & & & 0.993 & & & & & & & & 0.4174 & 2.0305 & & \\
\hline acle & 11 & 9.0 & 29050.0 & 10337.0 & 02.3 & 14139.4 & 95.2 & 10284.0 & 0.313 & -2.9 & $0 . c$ & 9.2967 & 0.003 & 91 & & 1.1022 & 0.3777 & 0. & 30 & 0. & 0.6783 & 10.7269 & 0 & 0.9577 \\
\hline & 20 & & & & & & & & & & & & 0.744 & & & & & & & & & & & \\
\hline Wahwe & 11 & 0.4 & 57 & 3.6 & 36.4 & .0 & & 1.0 & 76 & -1.5 & 0.393 & 3.5 & 0.141 & 91 & $0 . c$ & 31 & 0.4 & 41 & 1.6611 & 0 & 0. & 61 & 9 & 0.4335 \\
\hline 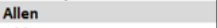 & 13 & 808.0 & 223 & & 5 & & & & 0.3 & -0.2 & & & & & & & & & & & & & & \\
\hline dma & 13 & 10.0 & & & .5 & & & 20 & 0.684 & -1.4499 & 0.437 & 1.0172 & 0.620 & 0.3077 & 0.4 & 0.8705 & 0.4549 & 0.1 & 63 & 0 & 0.3714 & 8 & & 0.4 \\
\hline . & 2 & & & & & & & & 0.77 & -1.6202 & 0.3 & 2.7817 & 0.220 & 0.5273 & & 1.4354 & 0.2 & & & & 0.5557 & 3.2 & & 0.5 \\
\hline & 9 & & & & & & & & 0.3 & & 0.8 & -0.2 & 0.804 & & & & & & & & 0. & 5 & & 0.3449 \\
\hline Dino & 14 & 27.7 & & & 0 & & & & & & 0.7 & & 0. & & & & & & & & & & & 10 \\
\hline Djado & of & 4.3 & & & & & & & & & & & & & & & & & & & & & & \\
\hline & 13 & 27.7 & 37468.5 & & 30.1 & & 0.0 & 5095.4 & 0.85 & & 0.6 & -0.2 & 0.830 & 0.6923 & 0.0 & 1.4919 & 0.2 & & 5 & 0 & 0.405 & 6 & 2 & 0.2152 \\
\hline & & 138.7 & & & & & & & & & & & & & & & & & & & & & & \\
\hline & 11 & 4.3 & 121 & & 40.0 & 2500.0 & 1100.8 & 2062.3 & 0. & & 0.5 & & 0.675 & & & & & & & & 111 & & & 0 \\
\hline & & 8650.0 & & & 9078.0 & & & & & & & & 0.111 & & & & & & & & & & & \\
\hline & 8 & & & & & & & & 0. & & 0.5 & -1 & & & & & 0. & & 1 & 0 & 78 & 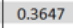 & 1.69 & \\
\hline & 3 & 45 & & & & & & & & & & & & & & & & & & & & & & \\
\hline & & & & & & & & & & & & & & & & & & & & & & & & \\
\hline & 13 & 15.2 & .2 & 5977.9 & 119.4 & 6097.3 & - & 2794.7 & 0. & -0.3 & 0.3 & -1.4 & 0.3 & 0. & 0.8 & 6267 & 0.2 & 0. & 0 & 0. & 0.3646 & & & 5 \\
\hline & & & & & & & & & & & & & & & & & & & & & & & & \\
\hline & 12 & 213.0 & & & & & & & & & 0.9 & & 0.5 & & & 9 & 0.4 & 0 & 1.2 & & 0.4533 & 1 & 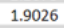 & , \\
\hline ollard & 5 & 213.0 & 8318.2 & 881.3 & 213.9 & 6095.2 & $2 \quad 342.0$ & 2592.1 & 0.60 & 0. & 0.104 & -3.0813 & 0.15 & & 0. & 1.2132 & 0.3466 & 0.2 & 8350 & 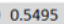 & 35 & .1768 & 1.2297 & c \\
\hline
\end{tabular}

Summary Statistics, KS Test and polynomial tests for herbivore guilds. $\alpha=0.05$, Sarle's $b=.555$ 


\section{Table S4.}

\begin{tabular}{|c|c|c|c|c|c|c|c|c|c|c|c|c|c|c|c|c|c|c|c|c|c|c|c|c|}
\hline \multirow{2}{*}{$\begin{array}{l}\text { Formation } \\
\text { Jurassic }\end{array}$} & \multirow[b]{2}{*}{$\mathrm{N}$} & \multicolumn{8}{|c|}{ Raw Data $=\log 2($ mass $(\mathrm{kg}))$} & \multicolumn{4}{|c|}{ M-S Distribution } & \multicolumn{2}{|c|}{\begin{tabular}{|l} 
KS Goodness of \\
Fit (vs Global)
\end{tabular}} & \multicolumn{3}{|c|}{$\begin{array}{c}\text { Quadratic Regression } \\
\text { (bins) }\end{array}$} & \multicolumn{3}{|c|}{$\begin{array}{c}\text { 4th degree polynomial } \\
\text { regression }\end{array}$} & \multicolumn{3}{|c|}{ Bimodality Coefficient } \\
\hline & & Min & $\operatorname{Max}$ & IQR & Q1 & $Q^{3}$ & Median & Mean & $P_{\text {Mean }}$ & Skew & $\mathbf{P}_{\text {Stew }}$ & kurtosis & $P_{\text {kurt }}$ & D & $\mathbf{P}$ & & & & & 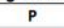 & R2 & b1 & b2 & b (Sarle) \\
\hline Lufeng BII "Zhangjia'ao" & & 9.7 & 324.8 & 315.1 & 9.7 & 324.8 & 25.0 & 119.8 & 0.391 & 1.2933 & 0.016 & & & 0.4424 & 0.0170 & 1.0215 & 0.4026 & 0.2034 & 0.7075 & 0.6154 & 0.3205 & & & \\
\hline Kayenta/Navajo Sandstone & 3 & 17.8 & 340.6 & 322.9 & 17.8 & 340.6 & 30.1 & 129.5 & 0.669 & 1.7321 & 0.004 & & & 0.4424 & 0.0170 & 0.6646 & 0.5407 & 0.1425 & 0.3818 & 0.8147 & 0.2029 & & & \\
\hline Hanson & 3 & 6.0 & 349.4 & 343.4 & 6.0 & 349.4 & 25.0 & 126.8 & 0.391 & 1.2933 & 0.016 & & & 0.4424 & 0.0170 & 1.0215 & 0.4026 & 0.2034 & .7075 & 0.6154 & 0.3205 & & & \\
\hline Xiashaximiao & 6 & 86.5 & 2950.0 & 1873.8 & 172.4 & 2046.3 & 225.5 & 905.6 & 0.170 & 0.4899 & 0.266 & -1.4667 & 0.442 & 0.2242 & 0.4720 & 1.6356 & 0.2538 & 0.2902 & 1.1923 & 0.4027 & 0.4429 & 0.3750 & 1.6400 & 0.1743 \\
\hline Shangshaximiao & 5 & 25.0 & 3000.0 & 2752.5 & 222.5 & 2975.0 & 1001.3 & 1479.3 & 0.034 & -1.9245 & 0.006 & 3.6667 & 0.006 & 0.3309 & 0.1270 & 0.8279 & 0.4712 & 0.1715 & .5481 & 0.7082 & 0.2676 & 6.5844 & 2.9167 & 0.6948 \\
\hline endaguru Middle Saurian & 9 & 10.7 & 2981.6 & 1527.5 & 131.7 & 1659.2 & 481.3 & 948.8 & 0.128 & -1.0103 & 0.190 & -0.5336 & 0.783 & 0.2216 & 0.4720 & 1.6278 & 0.2552 & 0.2892 & 8973 & 0.5200 & 0.3743 & 1.3331 & 2.1198 & 0.3487 \\
\hline endaguru Upper Saurian & 6 & 10.7 & 5700.0 & 2536.3 & 363.7 & 2900.0 & 1076.3 & 1718.5 & 0.012 & -1.7628 & 0.013 & 3.5586 & 0.006 & 0.3643 & 0.1270 & 1.1351 & 0.3682 & 0.2210 & .0644 & 0.4493 & 0.4151 & 4.8553 & 3.3629 & 0.6091 \\
\hline Morrison & 8 & 20.4 & 3758.7 & 2968.4 & 122.5 & 3090.9 & 1247.1 & 1496.9 & 0.034 & -0.5401 & 0.577 & -1.0500 & 0.783 & 0.1559 & 0.7580 & 4.4837 & 0.0494 & 0.5285 & 4725 & 0.0850 & 0.6983 & 0.3970 & 1.8333 & 0.2075 \\
\hline surinhã & 5 & 10.2 & 3483.3 & 2483.2 & 129.8 & 2613.0 & 580.4 & 1213.2 & 0.090 & -0.5901 & 0.513 & -0.0219 & 0.374 & 0.1840 & 0.7580 & 3.8576 & 0.0672 & 0.4909 & 3152 & 0.1714 & 0.6068 & 0.6191 & 1.9945 & 0.1620 \\
\hline \multicolumn{25}{|l|}{ Cretaceous } \\
\hline st Clay & 4 & 0.1 & 0.0 & 1464.3 & 3.5 & 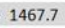 & .2 & 671.1 & 0.669 & 6 & $5 \mathrm{c}$ & . & 0.574 & 8 & 0 & 5 & 0 & 0 & 1 & 11 & ( & 31 & 1 & 10 \\
\hline xian & 9 & 0.6 & 20.0 & 6.3 & 1.3 & 7.6 & 3.1 & 5.2 & 0.000 & \begin{tabular}{|l|l|} 
& 0.8248 \\
\end{tabular} & 0.107 & -1.0794 & 0.760 & 0.7758 & 0.0000 & \begin{tabular}{|l|l|}
1.9772 \\
\end{tabular} & 0.2006 & 0.3308 & 98 & 0.0655 & 258 & 0.8885 & 1. & 0.2949 \\
\hline Ixian. & 12 & 1.1 & 1300.0 & 16.2 & 3.2 & 19.3 & 6.7 & 117.7 & 0.024 & 1.7714 & 0.004 & 4.0246 & 0.004 & 0.6091 & 0.0030 & 2.1738 & 0.1762 & 0.3521 & 13.7609 & 0.0035 & 0.9017 & 70 & & \\
\hline overly & 3 & 3.6 & 4558.8 & 4555.2 & 3.6 & 4558.8 & 83.2 & 1548.5 & 0.724 & \begin{tabular}{|l|l|} 
\\
\end{tabular} & 0.068 & & & 0. & 0.2 & 0.2955 & 0. & 0.0688 & & 0.8309 & 0.1923 & & & \\
\hline hure & 3 & 2.5 & 1742.7 & 1740.2 & 2.5 & 1742.7 & 10.8 & 585.4 & 0.391 & $\begin{array}{l}0.9352 \\
\end{array}$ & 0.068 & & & 0.4424 & 0.0170 & 0.3759 & 0.6982 & 0.0 & & 0.8309 & 0.1923 & & & \\
\hline edar & 8 & 5.8 & 3950.0 & 460.6 & 10.9 & 471.6 & 17.5 & 587.3 & 0.669 & \begin{tabular}{|l|}
1.4286 \\
\end{tabular} & 0.010 & 1.3571 & 0.055 & 0.4008 & 0.0500 & 1.0008 & 0.4093 & 0.2001 & & 0.5541 & 0.3550 & .7778 & 2.9796 & 0.4794 \\
\hline yyar & 7 & 13.3 & 1742.7 & 582.3 & 18.7 & 601.0 & 90.0 & 408.8 & 0.796 & 0.3830 & 0.345 & -2.1066 & 0.171 & 0.2437 & 0.4720 & 1.6756 & 0.2467 & 0.2 & & 0.5 & 0.3391 & 0.2112 & 1.3723 & 0.1788 \\
\hline $\mathrm{k}$ & 5 & 13.8 & 672.4 & 416.9 & 20.1 & 437.1 & 156.7 & 214.2 & 0.976 & -0 & 0.948 & -2.4074 & 0.106 & & 0.0500 & & & & & & & 38 & & 0.1116 \\
\hline sse & 7 & 1.4 & 220.0 & 2.8 & 10.2 & 13.0 & 12.4 & 40.1 & 0.105 & 00 & 0.717 & 3.0000 & 0.008 & 0.6329 & 0.0000 & & 0. & 0.1 & & 0.6622 & 140 & 0.0000 & 3 & 0.1124 \\
\hline inc & 6 & 2.8 & 1925.0 & 1340.1 & 3.7 & 1343.8 & 358.3 & 633.0 & 0.976 & & 0.923 & & 0.083 & & & & & & & & & 54 & & 0.1377 \\
\hline ilk R & 8 & 10.2 & 2554.8 & $\begin{array}{l}3 \\
3\end{array}$ & 10.8 & 23.7 & 19.3 & 7.2 & 0.308 & 84 & 0.000 & 8.0000 & 0.000 & 0 & 0.0000 & & 45 & 0 & & 0.8254 & 959 & 10.8889 & 6.1429 & 1.076 \\
\hline , & 3 & 672.4 & 232 & 1652.6 & 672.4 & 232 & 734.2 & 12 & 0.012 & 1 & 0.004 & & & & & & & & & & & & & \\
\hline vol & 8 & 5.8 & 2554.8 & 1882.7 & 11.0 & 1893.7 & 45.9 & & 0.796 & 70 & 0.064 & & 0.762 & & 0.1270 & & & & & 0.5261 & 08 & & & 0.32 \\
\hline lahw & 7 & 10.2 & 15 & 1453.7 & 13.0 & 14 & & & 0.920 & & & & 0.796 & & & & & & & & & & & \\
\hline ler & 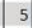 & 15.1 & .7 & 458.0 & 7.8 & 475.8 & 373.8 & & 0.622 & 86 & 0.497 & -3 & 0.041 & & 0.0500 & & & & & 0.8 & & & & 0. \\
\hline , the & 6 & 10.3 & & 678.0 & & 689.8 & & & 0.519 & & & & 0.004 & & & & & & & & & & & \\
\hline ip & 7 & 10.2 & 15 & 67.0 & 13.0 & 8 & 7 & & 0.427 & 66 & 0.000 & 5 & 0.003 & 0 & 0.0170 & & & 0.1 & & 0.7 & 44 & 97 & 4 & 10 \\
\hline 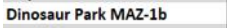 & 13 & 2.6 & & & 10.3 & & & & 0.427 & & & & 0.077 & & & & & & & & & & & \\
\hline Djadokh & 14 & 0.2 & 3103.1 & 19.9 & 2.4 & 22.3 & 16.2 & 0 & 0.012 & 32 & 0.097 & 2.5585 & 0.022 & 989 & 0.0000 & 29 & 59 & 0.1 & & 0.5 & & 45 & 19 & 0.2294 \\
\hline 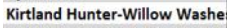 & 6 & 10.2 & & 763.4 & 12.7 & 77 & & & 0.519 & & & & 0.004 & & & & & & & & & & & \\
\hline anyon Drumheller & 9 & 10.3 & 2345.2 & 93.5 & 13.3 & 106.8 & .5 & & 0.572 & 08 & 0.004 & & 0.008 & 0 & 0.0500 & 62 & 29 & 0.2404 & & & & & 3 & 0.5950 \\
\hline 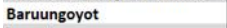 & 12 & 0.2 & & & 1.8 & & 1.6 & & 0.028 & & 0.085 & & 0.049 & 0. & 0.0070 & & & & & & & & & \\
\hline Lameta & 7 & 9.1 & 3500.0 & 2507.6 & 12.0 & 2519.6 & 491.3 & 1152.4 & 0.278 & -0.3718 & 0.770 & & 0.325 & & & & & & & & & & & \\
\hline Prince $C$ & 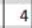 & 14.7 & 750.0 & 562.6 & 16.9 & 579.5 & 45.8 & 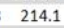 & 0.669 & 1.4142 & 0.011 & 1.5 & 0.0 & I & 0.0 & & 0. & & 7 & 0.4 & $c$ & 4.500 & 2.00 & \\
\hline & 3 & 0.8 & & & 0.8 & & & & & & 0.068 & & & & & & & & & & & & & \\
\hline & 4 & 9 & & 4814.9 & 9.9 & 482 & 3 & 161 & 0. & & 0. & & 0. & & & & & & & & & & & \\
\hline (II) & 9 & 10.2 & & & 4.4 & & & & & & & & & & & & & & & & & & & \\
\hline Tremp & & 0.2 & & 188.0 & 12.3 & 200.3 & 13. & 136 & & & 0.000 & & 0.0 & & & & 0 & & & 0.7 & & & 4 & \\
\hline & 11 & 2 & & 5 & 3.0 & & 3.4 & & & & & & & & & & & & & & & & & \\
\hline Scollar & 1. & 10.2 & 3.6 & 3236.8 & .6 & 3248.4 & 4.7 & 1306.9 & & 315 & 0.003 & 512 & & 160 & & 525 & 960 & 214 & 4500 & 7703 & & 7.3371 & 3.0378 & 7 \\
\hline
\end{tabular}

Summary Statistics, KS Test and polynomial tests for carnivore guilds. $\alpha=0.05$, Sarle's $b=.555$ 
Table S5.

\begin{tabular}{|c|c|c|c|c|c|c|c|c|c|c|c|c|c|}
\hline & \multirow[b]{2}{*}{$\log (\mathbf{k g})$} & \multicolumn{12}{|c|}{ Percentage of Communities Containing at least one Species } \\
\hline \multirow{5}{*}{$\overline{\bar{\alpha}}$} & & -1 & -0.5 & 0 & 0.5 & 1 & 1.5 & 2 & 2.5 & 3 & 3.5 & 4 & 4.5 \\
\hline & Carnivores & 2 & 5 & 21 & 28 & 88 & 47 & 23 & 42 & 63 & 23 & 0 & 0 \\
\hline & Herbivores & 0 & 0 & 14 & 23 & 42 & 37 & 63 & 53 & 67 & 79 & 37 & 14 \\
\hline & Carnivores with larger Carnivore & 2 & 5 & 21 & 28 & 86 & 47 & 19 & 26 & 9 & 0 & 0 & 0 \\
\hline & Herbivores with larger Herbivore & 0 & 0 & 14 & 23 & 40 & 37 & 63 & 49 & 65 & 35 & 5 & 0 \\
\hline \multirow{4}{*}{ 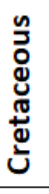 } & Carnivores & 2 & 5 & 21 & 23 & 67 & 42 & 12 & 21 & 44 & 16 & 0 & 0 \\
\hline & Herbivores & 0 & 0 & 12 & 16 & 33 & 28 & 49 & 35 & 53 & 60 & 23 & 7 \\
\hline & Carnivores with larger Carnivore & 2 & 5 & 21 & 23 & 65 & 42 & 9 & 12 & 2 & 0 & 0 & 0 \\
\hline & Herbivores with larger Herbivore & 0 & 0 & 12 & 16 & 30 & 28 & 49 & 35 & 53 & 21 & 0 & 0 \\
\hline \multirow{4}{*}{ 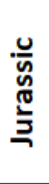 } & Carnivores & 0 & 0 & 0 & 5 & 21 & 5 & 12 & 21 & 19 & 7 & 0 & 0 \\
\hline & Herbivores & 0 & 0 & 2 & 7 & 9 & 9 & 14 & 19 & 14 & 19 & 14 & 7 \\
\hline & Carnivores with larger Carnivore & 0 & 0 & 0 & 5 & 21 & 5 & 9 & 14 & 7 & 0 & 0 & 0 \\
\hline & Herbivores with larger Herbivore & 0 & 0 & 2 & 7 & 9 & 9 & 14 & 14 & 12 & 14 & 5 & 0 \\
\hline
\end{tabular}

The likelihood of bin occupation by guild with and without larger species present. 
Table S6.

\begin{tabular}{|lrrr|}
\multicolumn{1}{c}{ Biomass Proportion } \\
\hline Species & Adult & Juvenile & RSP \\
\hline Acrocanthosaurus atokensis & 0.32 & 0.68 & $211 \%$ \\
\hline Albertosaurus sarcophagus & 0.31 & 0.69 & $219 \%$ \\
\hline Alectrosaurus olseni & 0.31 & 0.69 & $219 \%$ \\
\hline Alioramus altai & 0.31 & 0.69 & $217 \%$ \\
Allosaurus fragilis & 0.55 & 0.45 & $82 \%$ \\
\hline Daspletosaurus torosus & 0.63 & 0.37 & $60 \%$ \\
Gorgosaurus libratus & 0.40 & 0.60 & $148 \%$ \\
\hline Siats meekerorum & 0.32 & 0.68 & $211 \%$ \\
\hline Tarbosaurus bataar & 0.36 & 0.64 & $179 \%$ \\
\hline Tyrannosaurus rex & 0.40 & 0.60 & $148 \%$
\end{tabular}

Proportional biomass for 10 species of large theropods 
Table S7.

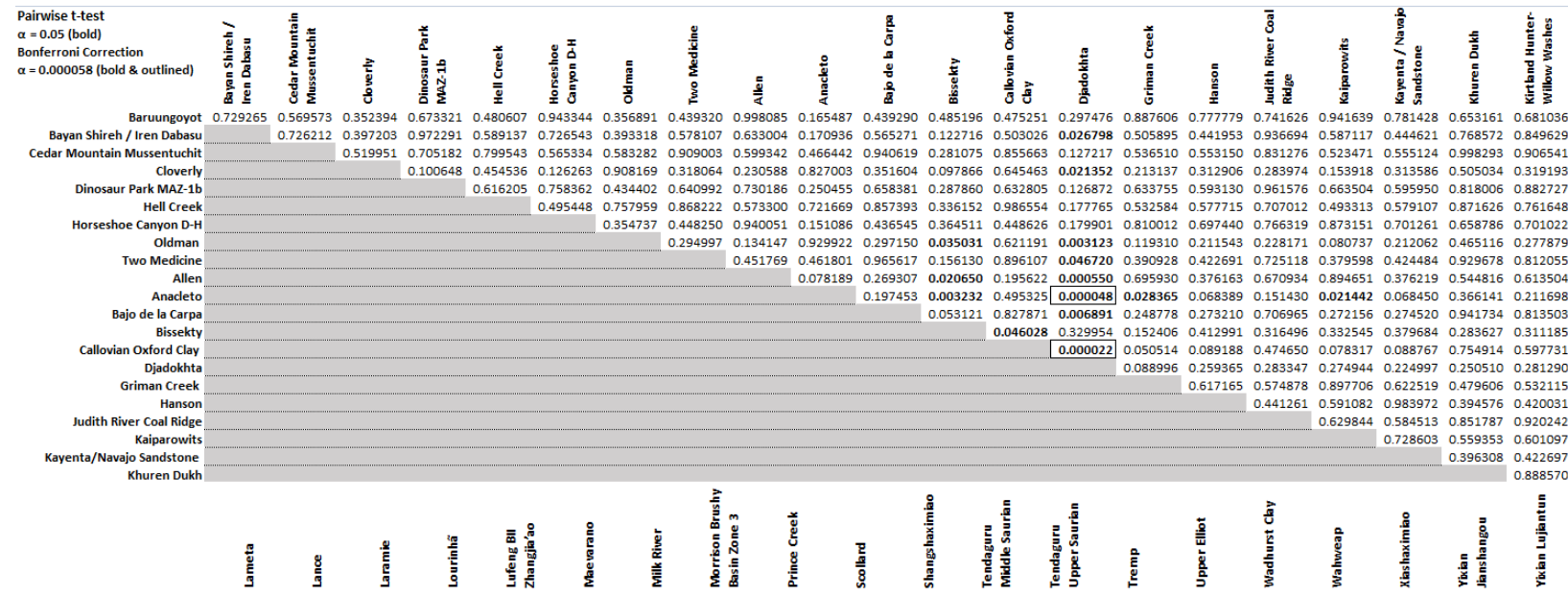

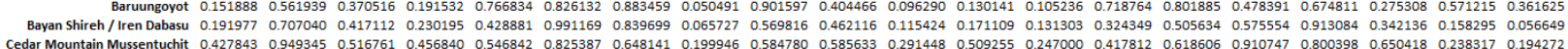

$\begin{array}{llllllllllllllllllllllll}\text { Cloverly } & 0.685267 & 0.300063 & 0.970736 & 0.773010 & 0.310670 & 0.418533 & 0.167609 & 0.955709 & 0.269784 & 0.876460 & 0.952114 & 0.458838 & 0.887555 & 0.151292 & 0.411741 & 0.468468 & 0.227351 & 0.530402 & 0.038924 & 0.054711\end{array}$

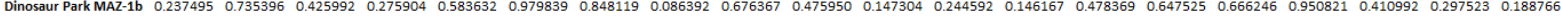

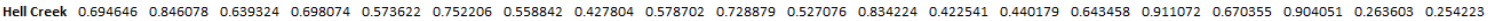
$\begin{array}{lllllllllllllllllllll}\text { Horseshoe Canyon D-H } & 0.150732 & 0.582807 & 0.378361 & 0.189815 & 0.684860 & 0.846191 & 0.927940 & 0.050044 & 0.834971 & 0.413276 & 0.094301 & 0.124146 & 0.107810 & 0.619278 & 0.731914 & 0.471649 & 0.697037 & 0.272768 & 0.448467 & 0.242804\end{array}$

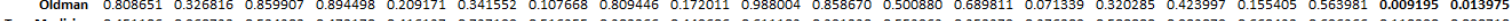

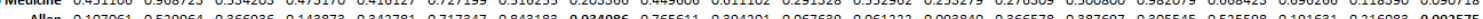

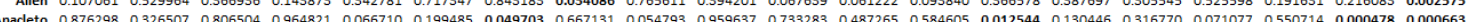

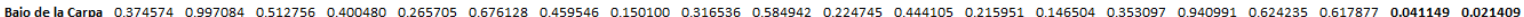
$\begin{array}{rllllllllllllllllllll}\text { Bujo de la Carpa } & 0.374574 & 0.997084 & 0.512756 & 0.400480 & 0.265705 & 0.676128 & 0.459546 & 0.150100 & 0.316536 & 0.584942 & 0.224745 & 0.444105 & 0.215951 & 0.146504 & 0.353097 & 0.940991 & 0.624235 & 0.617877 & 0.041149 & 0.021409 \\ \text { Bissekty } & 0.042956 & 0.301782 & 0.286120 & 0.068232 & 0.420487 & 0.297738 & 0.341519 & 0.012289 & 0.303296 & 0.294756 & 0.028903 & 0.013324 & 0.051354 & 0.426099 & 0.426638 & 0.102374 & 0.135281 & 0.075643 & 0.470513 & 0.177315\end{array}$

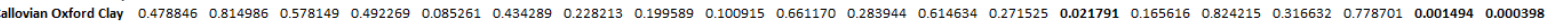
$\begin{array}{lllllllllllllllllllllll}\text { Djadokhta } 0.032941 & 0.280442 & 0.268264 & 0.053548 & 0.252880 & 0.254417 & 0.301173 & 0.008198 & 0.228091 & 0.277269 & 0.019693 & 0.008736 & 0.040697 & 0.312647 & 0.215375 & 0.078295 & 0107299 & 0.060107 & 0.347201 & 0.107440\end{array}$ $\begin{array}{llllllllllllllllllllll}\text { Griman Creek } & 0.089513 & 0.467570 & 0.348253 & 0.125013 & 0.581508 & 0.610220 & 0.704930 & 0.028216 & 0.999374 & 0.369960 & 0.058549 & 0.045859 & 0.083690 & 0.618222 & 0.614992 & 0.251333 & 0.407035 & 0.159696 & 0.448865 & 0.022857 & \end{array}$ $\begin{array}{lllllllllllllllllllllllllll}\text { Hanson } & 0.071672 & 0.381830 & 0.333442 & 0.108922 & 0.957783 & 0.456591 & 0.509440 & 0.023645 & 0.637118 & 0.343382 & 0.054680 & 0.029614 & 0.077546 & 0.940300 & 0.936196 & 0.184703 & 0.252562 & 0.123525 & 0.936711 & 0.043215\end{array}$ Judith River Coal Ridge $\begin{array}{lllllllllllllllllllllll}0.268549 & 0.769893 & 0.448039 & 0.308792 & 0.573105 & 0.948850 & 0.807274 & 0.106114 & 0.653535 & 0.498202 & 0.176093 & 0.282293 & 0.168980 & 0.459803 & 0.640067 & 0.709594 & 0.999074 & 0.449838 & 0.276769 & 0.184856\end{array}$

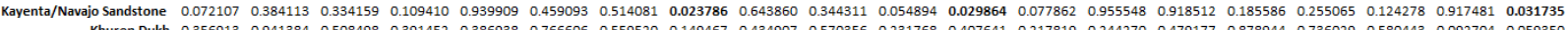

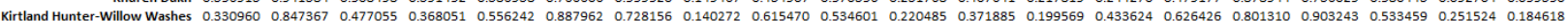

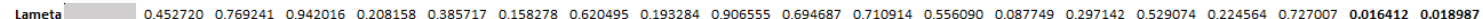
Lance $\quad \begin{array}{llllllllllllllllllll} & 0.564147 & 0.569874 & 0.639218 & 0.840759 & 0.682720 & 0.305348 & 0.661545 & 0.638700 & 0.408346 & 0.650529 & 0.326473 & 0.524233 & 0.699092 & 0.967817 & 0.805447 & 0.756151 & 0.352369 & 0.319817\end{array}$

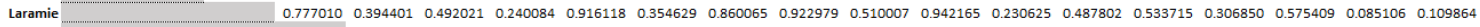

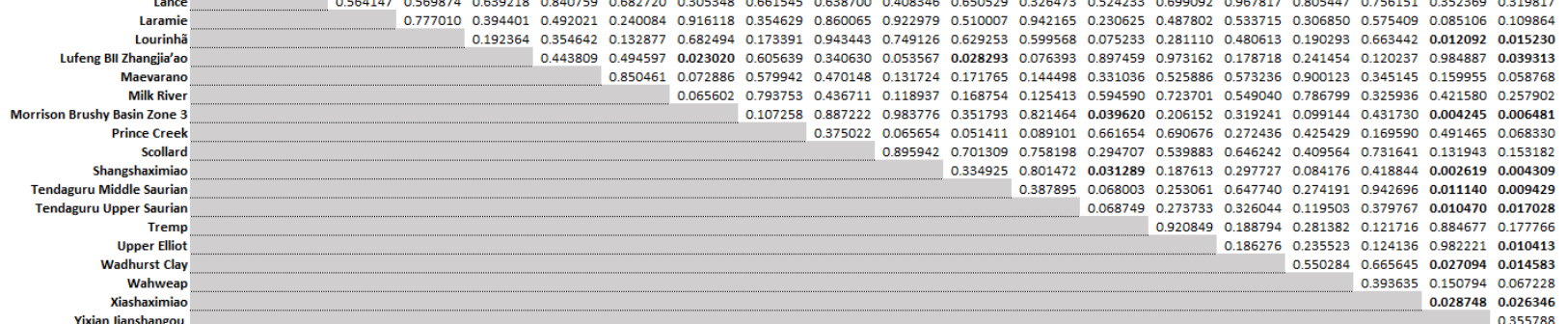

Pairwise t-test of communities with Bonferroni correction alpha value. 


\section{References and Notes}

1. S. C. Wang, P. Dodson, Estimating the diversity of dinosaurs. Proc. Natl. Acad. Sci. U.S.A. 103, 13601-13605 (2006). Medline

2. J. H. Brown, P. F. Nicoletto, Spatial scaling of species composition: Body masses of North American land mammals. Am. Nat. 138, 1478-1512 (1991). doi:10.1086/285297

3. D. A. Kelt, J. H. Brown, E. J. Heske, P. A. Marquet, S. R. Morton, J. R. W. Reid, K. A. Rogovin, G. Shenbrot, Community structure of desert small mammals: Comparisons across four continents. Ecology 77, 746-761 (1996). doi:10.2307/2265499

4. E. J. O'Gorman, D. W. E. Hone, Body size distribution of the dinosaurs. PLOS ONE 7, e51925 (2012). doi:10.1371/journal.pone.0051925 Medline

5. C. M. Brown, D. C. Evans, N. E. Campione, L. J. O’Brien, D. A. Eberth, Evidence for taphonomic size bias in the Dinosaur Park Formation (Campanian, Alberta), a model Mesozoic terrestrial alluvial-paralic system. Palaeogeogr. Palaeoclimatol. Palaeoecol. 372, 108-122 (2013). doi:10.1016/j.palaeo.2012.06.027

6. R. S. Seymour, Dinosaur eggs: gas conductance through the shell, water loss during incubation and clutch size. Paleobiology 5, 1-11 (1979). doi: $10.1017 / \mathrm{S} 0094837300006242$

7. N. O. Ratsimbaholison, R. N. Felice, P. M. O'Connor, Ontogenetic changes in the craniomandibular skeleton of the abelisaurid dinosaur Majungasaurus crenatissimus from the Late Cretaceous of Madagascar. Acta Palaeontol. Pol. 61, 281-292 (2016). doi:10.4202/app.00132.2014

8. T. D. Carr, Craniofacial ontogeny in Tyrannosauridae (Dinosauria, Coelurosauria). J. Vertebr. Paleontol. 19, 497-520 (1999). doi:10.1080/02724634.1999.10011161

9. D. Codron, C. Carbone, D. W. H. Müller, M. Clauss, Ontogenetic niche shifts in dinosaurs influenced size, diversity and extinction in terrestrial vertebrates. Biol. Lett. 8, 620-623 (2012). doi:10.1098/rsbl.2012.0240 Medline

10. E. E. Werner, J. F. Gilliam, The ontogenetic niche and species interactions in size-structured populations. Annu. Rev. Ecol. Syst. 15, 393-425 (1984). doi:10.1146/annurev.es.15.110184.002141

11. D. Codron, C. Carbone, M. Clauss, Ecological interactions in dinosaur communities: Influences of small offspring and complex ontogenetic life histories. PLOS ONE $\mathbf{8}$, e77110 (2013). doi:10.1371/journal.pone.0077110 Medline

12. H. N. Woodward, K. Tremaine, S. A. Williams, L. E. Zanno, J. R. Horner, N. Myhrvold, Growing up Tyrannosaurus rex: Osteohistology refutes the pygmy "Nanotyrannus" and supports ontogenetic niche partitioning in juvenile Tyrannosaurus. Sci. Adv. 6, eaax6250 (2020). doi:10.1126/sciadv.aax6250 Medline

13. D. Purwandana, A. Ariefiandy, M. J. Imansyah, A. Seno, C. Ciofi, M. Letnic, T. S. Jessop, Ecological allometries and niche use dynamics across Komodo dragon ontogeny. Naturwissenschaften 103, 27 (2016). doi:10.1007/s00114-016-1351-6 Medline

14. G. M. Erickson, K. C. Rogers, S. A. Yerby, Dinosaurian growth patterns and rapid avian 
growth rates. Nature 412, 429-433 (2001). doi:10.1038/35086558 Medline

15. J. R. Horner, A. De Ricqlès, K. Padian, Long bone histology of the hadrosaurid dinosaur Maiasaura peeblesorum: Growth dynamics and physiology based on an ontogenetic series of skeletal elements. J. Vertebr. Paleontol. 20, 115-129 (2000). doi:10.1671/02724634(2000)020[0115:LBHOTH]2.0.CO;2

16. G. M. Erickson, P. J. Currie, B. D. Inouye, A. A. Winn, Tyrannosaur life tables: An example of nonavian dinosaur population biology. Science 313, 213-217 (2006). doi:10.1126/science.1125721 Medline

17. G. M. Erickson, P. J. Currie, B. D. Inouye, A. A. Winn, A revised life table and survivorship curve for Albertosaurus sarcophagus based on the dry island mass death assemblage. Can. J. Earth Sci. 47, 1269-1275 (2010). doi:10.1139/E10-051

18. P. Gignac, H. O'Brien, Suchian feeding success at the interface of ontogeny and macroevolution. Integr. Comp. Biol. 56, 449-458 (2016). doi:10.1093/icb/icw041 Medline

19. D. J. Varricchio, A distinct dinosaur life history? Hist. Biol. 23, 91-107 (2011). doi:10.1080/08912963.2010.500379

20. The Paleobiology Database (2020); https://paleobiodb.org/.

21. Materials and methods are available as supplementary materials.

22. F. A. Smith, A. G. Boyer, J. H. Brown, D. P. Costa, T. Dayan, S. K. M. Ernest, A. R. Evans, M. Fortelius, J. L. Gittleman, M. J. Hamilton, L. E. Harding, K. Lintulaakso, S. K. Lyons, C. McCain, J. G. Okie, J. J. Saarinen, R. M. Sibly, P. R. Stephens, J. Theodor, M. D. Uhen, The evolution of maximum body size of terrestrial mammals. Science 330, 12161219 (2010). doi:10.1126/science.1194830 Medline

23. J. B. Foster, Evolution of mammals on Islands. Nature 202, 234-235 (1964). doi: $10.1038 / 202234 \mathrm{a} 0$

24. D. Codron, J. Codron, M. Sponheimer, M. Clauss, Within-population isotopic niche variability in savanna mammals: Disparity between carnivores and herbivores. Front. Ecol. Evol. 4, (2016). doi:10.3389/fevo.2016.00015

25. S. L. Brusatte, M. J. Benton, M. Ruta, G. T. Lloyd, Superiority, competition, and opportunism in the evolutionary radiation of dinosaurs. Science 321, 1485-1488 (2008). doi:10.1126/science.1161833 Medline

26. R. B. J. Benson, G. Hunt, M. T. Carrano, N. Campione, Cope's rule and the adaptive landscape of dinosaur body size evolution. Palaeontology 61, 13-48 (2017). doi:10.1111/pala.12329

27. R. B. J. Benson, N. E. Campione, M. T. Carrano, P. D. Mannion, C. Sullivan, P. Upchurch, D. C. Evans, Rates of dinosaur body mass evolution indicate 170 million years of sustained ecological innovation on the avian stem lineage. PLOS Biol. 12, e1001853 (2014). doi:10.1371/journal.pbio.1001853 Medline

28. K. T. Bates, R. B. J. Benson, P. L. Falkingham, A computational analysis of locomotor anatomy and body mass evolution in Allosauroidea (Dinosauria: Theropoda). 
Paleobiology 38, 486-507 (2016). doi:10.1666/10004.1

29. C. Foth, B. P. Hedrick, M. D. Ezcurra, Cranial ontogenetic variation in early saurischians and the role of heterochrony in the diversification of predatory dinosaurs. PeerJ 4, e1589 (2016). doi:10.7717/peerj. 1589 Medline

30. E. J. Rayfield, D. B. Norman, C. C. Horner, J. R. Horner, P. M. Smith, J. J. Thomason, P. Upchurch, Cranial design and function in a large theropod dinosaur. Nature 409, 10331037 (2001). doi:10.1038/35059070 Medline

31. R. Delcourt, Ceratosaur palaeobiology: New insights on evolution and ecology of the southern rulers. Sci. Rep. 8, 9730 (2018). doi:10.1038/s41598-018-28154-x Medline

32. J. O. Farlow, T. R. Holtz Jr., The fossil record of predation in dinosaurs. Paleontol. Soc. Pap. 8, 251-266 (2002). doi:10.1017/S108933260000111X

33. T. D. Carr, A high-resolution growth series of Tyrannosaurus rex obtained from multiple lines of evidence. PeerJ 8, e9192 (2020). doi:10.7717/peerj.9192

34. A. R. Fiorillo, R. A. Gangloff, Theropod teeth from the prince creek formation (Cretaceous) of northern Alaska, with speculations on arctic dinosaur paleoecology. J. Vertebr.

Paleontol. 20, 675-682 (2001). doi:10.1671/02724634(2000)020[0675:TTFTPC $] 2.0 . \mathrm{CO} ; 2$

35. M. J. Ryan, P. J. Currie, J. D. Gardner, M. K. Vickaryous, J. M. Lavigne, Baby hadrosaurid material associated with an unusually high abundance of Troodon teeth from the Horseshoe Canyon Formation, Upper Cretaceous, Alberta, Canada. GAIA 15, 123-133 (2000); doi:10.7939/R32B8VS51

36. S. Wang, J. Stiegler, R. Amiot, X. Wang, G. Du, J. M. Clark, X. Xu, Extreme ontogenetic changes in a ceratosaurian theropod. Curr. Biol. 27, 144-148 (2017). doi:10.1016/j.cub.2016.10.043 Medline

37. G. M. Erickson, P. J. Makovicky, P. J. Currie, M. A. Norell, S. A. Yerby, C. A. Brochu, Gigantism and comparative life-history parameters of tyrannosaurid dinosaurs. Nature 430, 772-775 (2004). doi:10.1038/nature02699 Medline

38. P. J. Bybee, A. H. Lee, E. T. Lamm, Sizing the Jurassic theropod dinosaur Allosaurus: Assessing growth strategy and evolution of ontogenetic scaling of limbs. J. Morphol. 267, 347-359 (2006). doi:10.1002/jmor.10406 Medline

39. D. Stiles, The ivory trade and elephant conservation. Environ. Conserv. 31, 309-321 (2005). doi:10.1017/S0376892904001614

40. J. C. Parish, P. M. Barrett, A reappraisal of the ornithischian dinosaur Amtosaurus magnus Kurzanov and Tumanova 1978, with comments on the status of A. archibaldi Averianov 2002. Can. J. Earth Sci. 41, 299-306 (2004). doi:10.1139/e03-101

41. N. E. Campione, D. C. Evans, Cranial growth and variation in edmontosaurs (Dinosauria: Hadrosauridae): implications for latest Cretaceous megaherbivore diversity in North America. PLOS ONE 6, e25186 (2011). doi:10.1371/journal.pone.0025186 Medline

42. S. C. R. Maidment, G. Wei, A review of the Late Jurassic stegosaurs (Dinosauria, Stegosauria) from the People's Republic of China. Geol. Mag. 143, 621-634 (2006). 
doi:10.1017/S0016756806002500

43. G. S. Paul, The Princeton Field Guide to Dinosaurs (Princeton Univ. Press, ed. 2, 2016).

44. F. E. Novas, F. L. Agnolín, S. Bandyopadhyay, Cretaceous theropods from India: A review of specimens described by Huene and Matley (1933). Rev. del Mus. Argentino Ciencias Nat. nueva Ser. 67-103 (2004). doi:10.22179/REVMACN.6.74

45. K. Carpenter, P. Galton, A photo documentation of bipedal ornithischian dinosaurs from the Upper Jurassic Morrison Formation, USA. Geol. Intermt. West 5, 167-207 (2018). doi:10.31711/giw.v5.pp167-207

46. D. B. Norman, A taxonomy of iguanodontians (Dinosauria: Ornithopoda) from the lower Wealden Group (Cretaceous: Valanginian) of southern England. Zootaxa 2489, 47 (2010). doi:10.11646/zootaxa.2489.1.3

47. D. Norman, On Asian ornithopods (Dinosauria: Ornithischia). 3. A new species of iguanodontid dinosaur. Zool. J. Linn. Soc. 122, 291-348 (2008). doi:10.1111/j.10963642.1998.tb02533.X

48. A. Prieto-Marquez, J. R. Wagner, Pararhabdodon isonensis and Tsintaosaurus spinorhinus: A new clade of Lambeosaurine hadrosaurids from Eurasia. Cretac. Res. 30, 1238-1246 (2009). doi:10.1016/j.cretres.2009.06.005

49. G. Funston, Caenagnathids of the Dinosaur Park Formation (Campanian) of Alberta, Canada: Anatomy, osteohistology, taxonomy, and evolution. Vertebr. Anat. Morphol. Palaeontol. 8, 105-153 (2020). doi:10.18435/vamp29362

50. D. W. Krause, S. D. Sampson, M. T. Carrano, P. M. O'Connor, Overview of the history of discovery, taxonomy, phylogeny, and biogeography of Majungasaurus crenatissimus (Theropoda: Abelisauridae) from the Late Cretaceous of Madagascar. J. Vertebr.

Paleontol. 27 (sup2), 1-20 (2007). doi:10.1671/02724634(2007)27[1:OOTHOD]2.0.CO;2

51. V. M. Arbour, "Systematics, evolution, and biogeography of the ankylosaurid dinosaurs," thesis, University of Alberta, Edmonton, Alberta, Canada (2014); https://doi.org/10.7939/R31N7XW06.

52. R. T. Bakker, M. Williams, P. J. Currie, Nanotyrannus, a new genus of pygmy tyrannosaur, from the latest Cretaceous of Montana. Hunteria 1, 1-30 (1988). doi:10.5281/zenodo.1037529

53. P. J. Currie, Cranial anatomy of tyrannosaurid dinosaurs from the Late Cretaceous of Alberta, Canada. Acta Palaeontol. Pol. 48, 191-226 (2003). doi:10.1017/CBO9780511608377.023

54. S. L. Brusatte, T. D. Carr, T. E. Williamson, T. R. Holtz Jr., D. W. E. Hone, S. A. Williams, Dentary groove morphology does not distinguish 'Nanotyrannus' as a valid taxon of tyrannosauroid dinosaur. Comment on: "Distribution of the dentary groove of theropod dinosaurs: Implications for theropod phylogeny and the validity of the genus Nanotyrannus Bakker et al., 1988. Cretac. Res. 65, 232-237 (2016). doi:10.1016/j.cretres.2016.02.007

55. T. D. Carr, A high-resolution growth series of Tyrannosaurus rex obtained from multiple 
lines of evidence. PeerJ 8, e9192 (2020). doi:10.7717/peerj.9192

56. T. D. Carr, T. E. Williamson, Diversity of late Maastrichtian Tyrannosauridae (Dinosauria: Theropoda) from western North America. Zool. J. Linn. Soc. 142, 479-523 (2004). doi:10.1111/j.1096-3642.2004.00130.x

57. T. R. Holtz Jr, Dinosaurs: The Most Complete, Up-To-Date Encyclopedia for Dinosaur Lovers of All Ages (Updated Internet Supplement) (Random House Books for Young Readers, 2007).

58. A. Averianov, H. D. Sues, Morphometric analysis of the teeth and taxonomy of the enigmatic theropod Richardoestesia from the Upper Cretaceous of Uzbekistan. J. Vertebr. Paleontol. 39, e1614941 (2019). doi:10.1080/02724634.2019.1614941

59. A. T. McDonald, A subadult specimen of Rubeosaurus ovatus (Dinosauria: Ceratopsidae), with observations on other ceratopsids from the Two Medicine Formation. PLOS ONE 6, e22710 (2011). doi:10.1371/journal.pone.0022710 Medline

60. J. A. Wilson, P. Upchurch, A revision of Titanosaurus lydekker (Dinosauria: Sauropoda), the first dinosaur genus with a 'Gondwanan' distribution. J. Syst. Palaeontology 1, 125-160 (2003). doi:10.1017/S1477201903001044

61. J. B. Scannella, J. R. Horner, 'Nedoceratops': An example of a transitional morphology. PLOS ONE 6, e28705 (2011). doi:10.1371/journal.pone.0028705 Medline

62. J. B. Scannella, J. R. Horner, Torosaurus Marsh, 1891, is Triceratops Marsh, 1889 (Ceratopsidae: Chasmosaurinae): Synonymy through ontogeny. J. Vertebr. Paleontol. 30, 1157-1168 (2010). doi:10.1080/02724634.2010.483632

63. L. Maiorino, A. A. Farke, T. Kotsakis, P. Piras, Is Torosaurus Triceratops? Geometric morphometric evidence of Late Maastrichtian ceratopsid dinosaurs. PLOS ONE 8, e81608 (2013). doi:10.1371/journal.pone.0081608 Medline

64. N. R. Longrich, D. J. Field, Torosaurus is not Triceratops: Ontogeny in chasmosaurine ceratopsids as a case study in dinosaur taxonomy. PLOS ONE 7, e32623 (2012). doi:10.1371/journal.pone.0032623 Medline

65. H. Xing, J. C. Mallon, M. L. Currie, Supplementary cranial description of the types of Edmontosaurus regalis (Ornithischia: Hadrosauridae), with comments on the phylogenetics and biogeography of Hadrosaurinae. PLOS ONE 12, e0175253 (2017). doi:10.1371/journal.pone.0175253 Medline

66. T. A. Tumanova, V. R. Alifanov, First record of stegosaur (Ornithischia, Dinosauria) from the Aptian-Albian of Mongolia. Paleontol. J. 52, 1771-1779 (2019). doi:10.1134/S0031030118140186

67. R. D. Juárez Valieri, J. A. Haro, L. E. Fiorelli, J. O. Calvo, A new hadrosauroid (Dinosauria: Ornithopoda) from the Allen Formation (Late Cretaceous) of Patagonia, Argentina. Rev. del Mus. Argentino Ciencias Nat. Nueva Ser. 12, 217-231 (2010). doi:10.22179/REVMACN.12.242

68. P. Cruzado-Caballero, R. A. Coria, Revisiting the hadrosaurid (Dinosauria: Ornithopoda) diversity of the Allen Formation: A re-evaluation of Willinakaqe salitralensis from Salitral Moreno, Río Negro Province, Argentina. Ameghiniana 53, 231-237 (2016). 
doi:10.5710/AMGH.25.09.2015.2943

69. T. Sekiya, X. Jin, W. Zheng, M. Shibata, Y. Azuma, A new juvenile specimen of Yunnanosaurus robustus (Dinosauria: Sauropodomorpha) from Early to Middle Jurassic of Chuxiong Autonomous Prefecture, Yunnan Province, China. Hist. Biol. 26, 252-277 (2013). doi:10.1080/08912963.2013.821702

70. A. Martinelli, A. Forasiepi, Late Cretaceous vertebrates from bajo de Santa Rosa (Allen Formation), Río Negro province, Argentina, with the description of a new sauropod dinosaur (Titanosauridae). Rev. Mus. Argent. Cienc. Nat. 6, 257-265 (2004). doi:10.22179/REVMACN.6.88

71. R. Molina-Pérez, A. Larramendi, Dinosaur Facts and Figures: The Sauropods and Other Sauropodomorphs (Princeton Univ. Press, 2020).

72. L. Salgado, C. Azpilicueta, A new saltasaurine dinosaur (Sauropoda, Titanosauridae) from the Rio Negro Province (Allen Formation, Upper Cretaceous), Patagonia, Argentina. Ameghiniana 37, 259-264 (2000).

73. F. Seebacher, A new method to calculate allometric length-mass relationships of dinosaurs. $J$. Vertebr. Paleontol. 21, 51-60 (2001). doi:10.1671/02724634(2001)021[0051:ANMTCA]2.0.CO;2

74. P. Cruzado-Caballero, J. Powell, Bonapartesaurus rionegrensis, a new hadrosaurine dinosaur from South America: Implications for phylogenetic and biogeographic relations with North America. J. Vertebr. Paleontol. 37, e1289381 (2017). doi:10.1080/02724634.2017.1289381

75. R. A. Coria, B. G. Riga, S. Casadio, A new hadrosaurid (Dinosauria, Ornithopoda) from Allen Formation, La Pampa Province, Argentina. Ameghiniana 49, 552-572 (2012). doi:10.5710/AMGH.9.4.2012.487

76. L. Salgado, R. A. Coria, C. M. Magalhaes Ribeiro, A. Garrido, R. Rogers, M. E. Simón, A. B. Arcucci, K. C. Rogers, A. P. Carabajal, S. Apesteguía, M. Fernández, R. A. García, M. Talevi, Upper Cretaceous dinosaur nesting sites of Río Negro (Salitral Ojo de Agua and Salinas de Trapalcó-Salitral de Santa Rosa), northern Patagonia, Argentina. Cretac. Res. 28, 392-404 (2007). doi:10.1016/j.cretres.2006.06.007

77. L. Salgado, R. A. Coria, The genus Aeolosaurus (Sauropoda, Titanosauridae) in the Allen Formation (Campanian-Maastrichtian) of Rio Negro Province, Argentina. Ameghiniana 30, 119-128 (1993).

78. J. Porfiri, J. Calvo, Panamericansaurus schroederi gen. nov. sp. nov. Un nuevo Sauropoda (Titanosauridae-Aeolosaurini) de la Provincia del Neuquén, Cretácico Superior de Patagonia, Argentina. Braz. Geo. J. 1, 100-115 (2010).

79. R. A. Garcia, L. Salgado, The titanosaur sauropods from the Late Campanian-Early Maastrichtian Allen Formation of Salitral Moreno, Río Negro, Argentina. Acta Palaeontol. Pol. 58, 269-284 (2013). doi:10.4202/app.2011.0055

80. G. V. Mazzetta, P. Christiansen, R. A. Fariña, Giants and bizarres: Body size of some Southern South American Cretaceous dinosaurs. Hist. Biol. 16, 71-83 (2004). doi:10.1080/08912960410001715132 
81. R. A. García, A giant tooth from the Late Cretaceous (middle Campanian-lower Maastrichtian) of Patagonia, Argentina: An enormous titanosaur or a large toothed titanosaur? Cretac. Res. 41, 82-85 (2013). doi:10.1016/j.cretres.2012.10.004

82. M. J. Benton, Prehistoric Life (Dorling Kindersley, 2012).

83. F. L. Agnolin, J. E. Powell, F. E. Novas, M. Kundrát, New alvarezsaurid (Dinosauria, Theropoda) from uppermost Cretaceous of north-western Patagonia with associated eggs. Cretac. Res. 35, 33-56 (2012). doi:10.1016/j.cretres.2011.11.014

84. F. E. Novas, D. Pol, J. I. Canale, J. D. Porfiri, J. O. Calvo, A bizarre Cretaceous theropod dinosaur from Patagonia and the evolution of Gondwanan dromaeosaurids. Proc. Biol. Sci. 276, 1101-1107 (2008). doi:10.1098/rspb.2008.1554 Medline

85. R. A. Coria, in Mesozoic Vertebrate Life, D. H. Tanke, K. Carpenter, Eds. (Indiana Univ. Press, 2001), pp. 3-9.

86. R. Molina-Pérez, A. Larramendi, Dinosaur Facts and Figures: The Theropods and Other Dinosauriformes (Princeton Univ. Press, 2020).

87. H. A. Leanza, S. Apesteguía, F. E. Novas, M. S. de la Fuente, Cretaceous terrestrial beds from the Neuquén Basin (Argentina) and their tetrapod assemblages. Cretac. Res. 25, 6187 (2004). doi:10.1016/j.cretres.2003.10.005

88. R. A. Coria, L. S. Filippi, L. M. Chiappe, R. García, A. B. Arcucci, Overosaurus paradasorum gen. et sp. nov., a new sauropod dinosaur (Titanosauria: Lithostrotia) from the late Cretaceous of Neuquén, Patagonia, Argentina. Zootaxa 3683, 357-376 (2013). doi:10.11646/zootaxa.3683.4.2 Medline

89. L. S. Filippi, A. C. Garrido, Pitekunsaurus macayai gen. et sp. nov., nuevo titanosaurio (Saurischia, Sauropoda) del Cretácico Superior de la Cuenca Neuquina, Argentina. Ameghiniana 43, 575-590 (2008).

90. L. S. Filippi, R. A. García, A. C. Garrido, A new titanosaur sauropod dinosaur from the Upper Cretaceous of North Patagonia, Argentina. Acta Palaeontol. Pol. 56, 505-520 (2011). doi:10.4202/app.2010.0019

91. L. Salgado, R. A. Coria, Barrosasaurus casamiquelai gen. et sp. nov., a new titanosaur (Dinosauria, Sauropoda) from the anacleto formation (Late Cretaceous: Early Campanian) of Sierra Barrosa (Neuquén, Argentina). Zootaxa 2222, 1-16 (2009). doi:10.11646/zootaxa.2222.1.1

92. G. Grellet-Tinner, V. Codrea, A. Folie, A. Higa, T. Smith, First evidence of reproductive adaptation to "island effect" of a dwarf Cretaceous Romanian titanosaur, with embryonic integument in ovo. PLOS ONE 7, e32051 (2012). doi:10.1371/journal.pone.0032051 Medline

93. P. C. Sereno, R. N. Martinez, J. A. Wilson, D. J. Varricchio, O. A. Alcober, H. C. E. Larsson, Evidence for avian intrathoracic air sacs in a new predatory dinosaur from Argentina. PLOS ONE 3, e3303 (2008). doi:10.1371/journal.pone.0003303 Medline

94. P. Cruzado-Caballero, L. S. Filippi, A. H. Méndez, A. C. Garrido, I. Díaz-Martínez, First ornithopod remains from the Bajo de la Carpa Formation (Santonian, Upper Cretaceous), northern Patagonia, Argentina. Cretac. Res. 83, 182-193 (2018). 
doi:10.1016/j.cretres.2017.07.022

95. P. Cruzado-Caballero, A. H. Mendez, L. S. Filippi, R. D. Juárez Valieri, A. C. Garrido, paper presented at the VII Jornadas Internaciones sobre Paleontología de Dinosaurios y su Entorno, Bargos, Spain, 8 to 10 September 2016.

96. A. G. Martinelli, E. I. Vera, Achillesaurus manazzonei, a new alvarezsaurid theropod (Dinosauria) from the Late Cretaceous Bajo de la Carpa Formation, Río Negro Province, Argentina. Zootaxa 1582, 1-17 (2007). doi:10.11646/zootaxa.1582.1.1

97. J. D. Porfiri, R. D. Juárez Valieri, D. D. D. Santos, M. C. Lamanna, A new megaraptoran theropod dinosaur from the Upper Cretaceous Bajo de la Carpa Formation of northwestern Patagonia. Cretac. Res. 89, 302-319 (2018).

doi:10.1016/j.cretres.2018.03.014

98. M. D. Ezcurra, A. H. Méndez, First report of a derived abelisaurid theropod from the Bajo de la Carpa Formation (Late Cretaceous), Patagonia, Argentina. Bull. Geosci. 547-554 (2009). doi:10.3140/bull.geosci.1106

99. N. R. Longrich, P. J. Currie, D. Zhi-Ming, A new oviraptorid (dinosauria: Theropoda) from the Upper Cretaceous of Bayan Mandahu, Inner Mongolia. Palaeontology 53, 945-960 (2010). doi:10.1111/j.1475-4983.2010.00968.x

100. T. Jerzykiewicz, D. A. Russell, Late Mesozoic stratigraphy and vertebrates of the Gobi Basin. Cretac. Res. 12, 345-377 (1991). doi:10.1016/0195-6671(91)90015-5

101. V. M. Arbour, P. J. Currie, D. Badamgarav, The ankylosaurid dinosaurs of the Upper Cretaceous Baruungoyot and Nemegt formations of Mongolia. Zool. J. Linn. Soc. 172, 631-652 (2014). doi:10.1111/zoj.12185

102. S. M. Kurzanov, A. F. Bannikov, A new sauropod from the Upper Cretaceous of Mongolia. Paleo. J 17, 91-97 (1983).

103. V. R. Alifanov, R. Barsbold, Ceratonykus oculatus gen. et sp. nov., a new dinosaur (?Theropoda, Alvarezsauria) from the Late Cretaceous of Mongolia. Paleontol. J. 43, 94106 (2009). doi:10.1134/S0031030109010109

104. D. B. Weishampel, P. Dodson, H. Osmólska, The Dinosauria (Univ. of California Press, ed. 2, 2007).

105. H. Osmólska, Hulsanpes perlei n.g. n.sp. (Deinonychosauria, Saurischia, Dinosauria) from the Upper Cretaceous Barun Goyot Formation of Mongolia. Neues Jahrb. für Geol. und Paläontologie Monatshefte 7, 440-448 (1982). doi:10.1127/njgpm/1982/1982/440

106. J. Nakajima, Y. Kobayashi, T. Chinzorig, T. Tanaka, R. Takasaki, K. Tsogtbaatar, P. J. Currie, A. R. Fiorillo, Dinosaur tracks at the Nemegt locality: Paleobiological and paleoenvironmental implications. Palaeogeogr. Palaeoclimatol. Palaeoecol. 494, 147159 (2018). doi:10.1016/j.palaeo.2017.10.026

107. B. Rinchen, T. Khishigjav, paper presented at the 12th Symposium on Mesozoic Terrestrial Ecosystems, Shenyan, China, 16 to 20 August 2015.

108. T. Maryańska, H. Osmólska, Protoceratopsidae (Dinosauria) of Asia. Palaeontol. Pol. 33, 133-182 (1975). 
109. M. Watabe, K. Tsogtbaatar, R. M. Sullivan, A new pachycephalosaurid from the Baynshire Formation (Cenomanian-late Santonian), Gobi Desert, Mongolia. New Mex. Museum Nat. Hist. Sci. Bull. 53, 489-495 (2011).

110. A. O. Averianov, An ankylosaurid (Ornithischia: Ankylosauria) braincase from the Upper Cretaceous Bissekty formation of Uzbekistan. Bull. l'Institut R. des Sci. Nat. Belqique, Sci. la Terre (2002).

111. D. H. Tanke, M. K. Brett-Surman, in Mesozoic Vertebrate Life, D. H. Tanke, K. Carpenter, Eds. (Indiana Univ. Press, 2001), pp. 206-218.

112. J. Van Itterbeeck, D. J. Horne, P. Bultynck, N. Vandenberghe, Stratigraphy and palaeoenvironment of the dinosaur-bearing Upper Cretaceous Iren Dabasu Formation, Inner Mongolia, People's Republic of China. Cretac. Res. 26, 699-725 (2005). doi:10.1016/j.cretres.2005.03.004

113. K. Tsogtbaatar, D. B. Weishampel, D. C. Evans, M. Watabe, A new hadrosauroid (Dinosauria: Ornithopoda) from the Late Cretaceous Baynshire Formation of the Gobi Desert (Mongolia). PLOS ONE 14, e0208480 (2019). doi:10.1371/journal.pone.0208480 $\underline{\text { Medline }}$

114. T. Tsuihiji, M. Watabe, R. Barsbold, K. Tsogtbaatar, A gigantic caenagnathid oviraptorosaurian (Dinosauria: Theropoda) from the Upper Cretaceous of the Gobi Desert, Mongolia. Cretac. Res. 56, 60-65 (2015). doi:10.1016/j.cretres.2015.03.007

115. X. Xu, Q. Tan, J. Wang, X. Zhao, L. Tan, A gigantic bird-like dinosaur from the Late Cretaceous of China. Nature 447, 844-847 (2007). doi:10.1038/nature05849 Medline

116. D. T. Ksepka, M. A. Norell, Erketu Ellisoni, a Long-necked Sauropod from Bor Guvé (Dornogov Aimag, Mongolia). Am. Mus. Novit. 3508, 1 (2006). doi:10.1206/00030082(2006)3508[1:EEALSF]2.0.CO;2

117. A. Perle, M. A. Norell, J. M. Clark, “A new maniraptoran theropod, Achillobator giganticus (Dromaeosauridae), from the Upper Cretaceous of Burkhant, Mongolia: Contributions of the Department of Geology" (National University of Mongolia, 1999); pp. 1-105.

118. H. D. Sues, A. Averianov, A new basal hadrosauroid dinosaur from the Late Cretaceous of Uzbekistan and the early radiation of duck-billed dinosaurs. Proc. Biol. Sci. 276, 25492555 (2009). doi:10.1098/rspb.2009.0229 Medline

119. H. D. Sues, A. Averianov, Ornithomimidae (Dinosauria: Theropoda) from the Bissekty Formation (Upper Cretaceous: Turonian) of Uzbekistan. Cretac. Res. 57, 90-110 (2016). doi:10.1016/j.cretres.2015.07.012

120. L. P. Tatarinov, L. A. Nessov, "Dinosaurs of the Northern Eurasia: New data about assemblages, ecology, and palaeobiogeography" (St. Petersburg: St. Petersburg State University, Institute of the Earth Crust, 1996).

121. A. Averianov, H. D. Sues, Review of Cretaceous sauropod dinosaurs from Central Asia. Cretac. Res. 69, 184-197 (2017). doi:10.1016/j.cretres.2016.09.006

122. P. J. Currie, S. J. Godfrey, L. Nessov, New caenagnathid (Dinosauria: Theropoda) specimens from the Upper Cretaceous of North America and Asia. Can. J. Earth Sci. 30, 2255-2272 (1993). doi:10.1139/e93-196 
123. H. D. Sues, A. Averianov, Enigmatic teeth of small theropod dinosaurs from the Upper Cretaceous (Cenomanian-Turonian) of Uzbekistan. Can. J. Earth Sci. 50, 306-314 (2013). doi:10.1139/e2012-033

124. S. M. Kurzanov, Brain-case structure in the carnosaur Itemirus n. gen. and some aspects of the cranial anatomy of dinosaurs. Paleo. J. 3, 361-369 (1976).

125. S. L. Brusatte, A. Averianov, H. D. Sues, A. Muir, I. B. Butler, New tyrannosaur from the mid-Cretaceous of Uzbekistan clarifies evolution of giant body sizes and advanced senses in tyrant dinosaurs. Proc. Natl. Acad. Sci. U.S.A. 113, 3447-3452 (2016). doi:10.1073/pnas.1600140113 Medline

126. D. M. Martill, A review of the terrestrial vertebrate fossils of the Oxford Clay (CallovianOxfordian) of England. Mercian Geol. 11, 171-190 (1988).

127. R. Lydekker, On the jaw of a new carnivorous dinosaur from the Oxford clay of Peterborough. Q. J. Geol. Soc. Lond. 49, 284-287 (1893). doi:10.1144/GSL.JGS.1893.049.01-04.46

128. J. W. Hulke, Note on some dinosaurian remains in the collection of A. Leeds, Esq., of Eyebury, Northamptonshire. Q. J. Geol. Soc. Lond. 43, 695-702 (1887). doi:10.1144/GSL.JGS.1887.043.01-04.52

129. A. J. Charig, in Aspects of Vertebrate History: Essays in Honor of Edwin Harris Colbert, L. L. Jacobs, Ed. (Museum of Northern Arizona Press, 1980), pp. 19-33.

130. J. B. Delair, The dinosaurs of Wiltshire. Wiltsh. Archaeol. Nat. Hist. Mag. 68, 1-7 (1973). doi:10.1098/rstb.1964.0009

131. A. D. Walker, Triassic reptiles from the Elgin area: Ornithosuchus and the origin of carnosaurs. Philos. Trans. R. Soc. Lond. B. Biol. Sci. 744, 53-134 (1964). doi:10.1098/rstb.1964.0009

132. F. Von Huene, Carnivorous Saurischia in Europe since the Triassic. Bull. Geol. Soc. Am. 34, 449-458 (1923). doi:10.1130/GSAB-34-449

133. B. J. Chinnery, T. R. Lipka, J. I. Kirkland, M. J. Parrish, M. K. Brett-Surman, in Lower and Middle Cretaceous Terrestrial Ecosystems: New Mexico Museum of Natural History and Science Bulletin No. 14., S. G. Lucas, J. I. Kirkland, J. W. Estep, Eds. (New Mexico Museum of Natural History and Science, 1998), pp. 297-302.

134. A. A. Farke, W. D. Maxwell, R. L. Cifelli, M. J. Wedel, A ceratopsian dinosaur from the lower cretaceous of Western North America, and the biogeography of Neoceratopsia. PLOS ONE 9, e112055 (2014). doi:10.1371/journal.pone.0112055 Medline

135. R. L. Cifelli, R. L. Nydam, J. D. Gardner, A. Weil, J. G. Eaton, J. I. Kirkland, S. K. Madsen, in Vertebrate Paleontology in Utah (Miscellaneous Publication 99-1, Utah Geological Survey, 1999), pp. 201-218.

136. J. I. Kirkland, M. Suarez, C. Suarez, R. Hunt-Foster, The Lower Cretaceous in east-central Utah: The Cedar Mountain Formation and its bounding strata. Geol. Intermt. West $\mathbf{3}$, 101-228 (2016). doi:10.31711/giw.v3.pp101-228

137. K. Carpenter, J. I. Kirkland, D. L. Burge, J. Bird, in Vertebrate Paleontology in Utah 
(Miscellaneous Publication 99-1, Utah Geological Survey, 1999), pp. 243-252.

138. K. Carpenter, Y. Ishida, Early and "middle" Cretaceous iguanodonts in time and space. J. Iber. Geol. 36, 145-164 (2010). doi:10.5209/rev_JIGE.2010.v36.n2.3

139. J. R. Garrison Jr., D. Brinkman, D. J. Nichols, P. Layer, D. Burge, D. Thayn, A multidisciplinary study of the Lower Cretaceous Cedar Mountain Formation, Mussentuchit Wash, Utah: A determination of the paleoenvironment and paleoecology of the Eolambia caroljonesa dinosaur quarry. Cretac. Res. 28, 461-494 (2007). doi:10.1016/j.cretres.2006.07.007

140. K. Carpenter, J. Bartlett, J. Bird, R. Barrick, Ankylosaurs from the Price River Quarries, Cedar Mountain Formation (Lower Cretaceous), east-central Utah. J. Vertebr. Paleontol. 28, 1089-1101 (2008). doi:10.1671/0272-4634-28.4.1089

141. R. D. Conkwright, "Maryland's State Dinosaur (Astrodon johnstoni)” (Maryland Geological Survey, 1998); www.mgs.md.gov/geology/fossils/maryland_state_dinosaur.html.

142. J. A. Frederickson, M. H. Engel, R. L. Cifelli, Niche partitioning in theropod dinosaurs: Diet and habitat preference in predators from the Uppermost Cedar Mountain Formation (Utah, U.S.A.). Sci. Rep. 8, 17872 (2018). doi:10.1038/s41598-018-35689-6 Medline

143. L. E. Zanno, P. J. Makovicky, Neovenatorid theropods are apex predators in the Late Cretaceous of North America. Nat. Commun. 4, 2827 (2013). doi:10.1038/ncomms3827 Medline

144. M. P. J. Oreska, M. T. Carrano, K. M. Dzikiewicz, Vertebrate paleontology of the Cloverly Formation (Lower Cretaceous). I: Faunal composition, biogeographic relationships, and sampling. J. Vertebr. Paleontol. 33, 264-292 (2013). doi:10.1080/02724634.2012.717567

145. W. L. Parsons, K. M. Parsons, A new ankylosaur (Dinosauria: Ankylosauria) from the Lower Cretaceous Cloverly Formation of central Montana. Can. J. Earth Sci. 46, 721738 (2009). doi:10.1139/E09-045

146. D. C. Woodruff, A new titanosauriform from the Early Cretaceous Cloverly Formation of Montana. Cretac. Res. 36, 58-66 (2012). doi:10.1016/j.cretres.2012.02.003

147. M. J. Wedel, R. L. Cifelli, R. K. Sanders, Osteology, paleobiology, and relationships of the sauropod dinosaur Sauroposeidon. Acta Palaeontol. Pol. 45, 343-388 (2000).

148. R. M. Sullivan, Revision of the dinosaur Stegoceras lambe (Ornithischia, Pachycephalosauridae). J. Vertebr. Paleontol. 23, 181-207 (2003). doi:10.1671/02724634(2003)23[181:ROTDSL]2.0.CO;2

149. D. A. Eberth, D. C. Evans, D. B. Brinkman, F. Therrien, D. H. Tanke, L. S. Russell, Dinosaur biostratigraphy of the Edmonton group (upper Cretaceous), Alberta, Canada: Evidence for climate influence. Can. J. Earth Sci. 50, 701-726 (2013). doi:10.1139/cjes$\underline{2012-0185}$

150. J. C. Mallon, Competition structured a Late Cretaceous megaherbivorous dinosaur assemblage. Sci. Rep. 9, 15447 (2019). doi:10.1038/s41598-019-51709-5 Medline

151. J. C. Mallon, D. C. Evans, M. J. Ryan, J. S. Anderson, Megaherbivorous dinosaur turnover 
in the Dinosaur Park Formation (Upper Campanian) of Alberta, Canada. Palaeogeogr. Palaeoclimatol. Palaeoecol. 350-352, 124-138 (2012). doi:10.1016/j.palaeo.2012.06.024

152. N. R. Longrich, P. J. Currie, A microraptorine (Dinosauria-Dromaeosauridae) from the Late Cretaceous of North America. Proc. Natl. Acad. Sci. U.S.A. 106, 5002-5007 (2009). doi:10.1073/pnas.0811664106 Medline

153. D. W. Larson, P. J. Currie, Multivariate analyses of small theropod dinosaur teeth and implications for paleoecological turnover through time. PLOS ONE 8, e54329 (2013). doi:10.1371/journal.pone.0054329 Medline

154. G. F. Funston, P. J. Currie, New material of Chirostenotes pergracilis (Theropoda, Oviraptorosauria) from the Campanian Dinosaur Park Formation of Alberta, Canada. Hist. Biol. 10.1080/08912963.2020.1726908 (2020). doi:10.1080/08912963.2020.1726908

155. G. F. Funston, W. S. Persons, G. J. Bradley, P. J. Currie, New material of the large-bodied caenagnathid Caenagnathus collinsi from the Dinosaur Park Formation of Alberta, Canada. Cretac. Res. 54, 179-187 (2015). doi:10.1016/j.cretres.2014.12.002

156. V. M. Arbour, M. E. Burns, R. L. Sissons, A redescription of the ankylosaurid dinosaur Dyoplosaurus acutosquameus Parks, 1924 (Ornithischia: Ankylosauria) and a revision of the genus. J. Vertebr. Paleontol. 29, 1117-1135 (2010). doi:10.1671/039.029.0405

157. Ł. Czepiński, Ontogeny and variation of a protoceratopsid dinosaur Bagaceratops rozhdestvenskyi from the Late Cretaceous of the Gobi Desert. Hist. Biol. 32, 1394-1421 (2019). doi:10.1080/08912963.2019.1593404

158. T. Chinzorig, Y. Kobayashi, K. Tsogtbaatar, P. J. Currie, M. Watabe, R. Barsbold, First Ornithomimid (Theropoda, Ornithomimosauria) from the Upper Cretaceous Djadokhta Formation of Tögrögiin Shiree, Mongolia. Sci. Rep. 7, 5835 (2017). Medline

159. L. M. Chiappe, M. A. Norell, J. M. Clark, in Mesozoic Birds: Above the Heads of Dinosaurs, L. M. Chiappe, L. M. Witmer, Eds. (Univ. of California Press, 2002), pp. 87120.

160. A. H. Turner, D. Pol, M. A. Norell, Anatomy of Mahakala omnogovae (Theropoda: Dromaeosauridae), Tögr ögiin Shiree, Mongolia. Am. Mus. Novit. 3722, 1-66 (2011). doi:10.1206/3722.2

161. A. Cau, V. Beyrand, D. F. A. E. Voeten, V. Fernandez, P. Tafforeau, K. Stein, R. Barsbold, K. Tsogtbaatar, P. J. Currie, P. Godefroit, Synchrotron scanning reveals amphibious ecomorphology in a new clade of bird-like dinosaurs. Nature 552, 395-399 (2017). doi:10.1038/nature24679 Medline

162. M. H. Schweitzer, J. A. Watt, R. Avci, L. Knapp, L. Chiappe, M. Norell, M. Marshall, Betakeratin specific immunological reactivity in feather-like structures of the Cretaceous Alvarezsaurid, Shuvuuia deserti. J. Exp. Zool. 285, 146-157 (1999). doi:10.1002/(SICI)1097-010X(19990815)285:2<146:AID-JEZ7>3.0.CO;2-A Medline

163. P. J. Makovicky, M. A. Norell, J. M. Clark, T. B. Rowe, Osteology and relationships of Byronosaurus jaffei (Theropoda: Troodontidae). Am. Mus. Novit. 3402, 1-32 (2003). doi:10.1206/0003-0082(2003)402<0001:OAROBJ>2.0.CO;2 
164. A. H. Turner, S. J. Nesbitt, M. A. Norell, A large alvarezsaurid from the Late Cretaceous of Mongolia. Am. Mus. Novit. 3648, 1-14 (2009). doi:10.1206/639.1

165. M. A. Norell, J. M. Clark, A. H. Turner, P. J. Makovicky, R. Barsbold, T. Rowe, A new dromaeosaurid theropod from Ukhaa Tolgod (Ömnögov, Mongolia). Am. Mus. Novit. 3545, 1 (2006). doi:10.1206/0003-0082(2006)3545[1:ANDTFU]2.0.CO;2

166. J. M. Clark, M. A. Norell, R. Barsbold, Two new oviraptorids (Theropoda: Oviraptorosauria), Upper Cretaceous Djadokhta Formation, Ukhaa Tolgod, Mongolia. $J$. Vertebr. Paleontol. 21, 209-213 (2001). doi:10.1671/02724634(2001)021[0209:TNOTOU]2.0.CO;2

167. F. v. Huene, The fossil reptile order Saurischia, their development and history. Monogr. zur Geol. und Palaeontol. 4, 1-361 (1932).

168. P. R. Bell, F. Fanti, L. J. Hart, L. A. Milan, S. J. Craven, T. Brougham, E. Smith, Revised geology, age, and vertebrate diversity of the dinosaur-bearing Griman Creek Formation (Cenomanian), Lightning Ridge, New South Wales, Australia. Palaeogeogr. Palaeoclimatol. Palaeoecol. 514, 655-671 (2019). doi:10.1016/j.palaeo.2018.11.020

169. P. R. Bell, M. C. Herne, T. Brougham, E. T. Smith, Ornithopod diversity in the Griman Creek Formation (Cenomanian), New South Wales, Australia. PeerJ 6, e6008 (2018). doi:10.7717/peerj.6008 Medline

170. R. E. Molnar, in Vertebrate Paleontology of Australasia, P. Vickers-Rich, J. M. Monaghan, R. F. Baird, T. H. Rich, Eds. (Pioneer Design Studio; Monash University Publications Committee, 1991), pp. 605-702. doi:10.5962/bhl.title.60647

171. F. L. Agnolin, M. D. Ezcurra, D. F. Pais, S. W. Salisbury, A reappraisal of the cretaceous non-avian dinosaur faunas from Australia and New Zealand: Evidence for their Gondwanan affinities. J. Syst. Palaeontology 8, 257-300 (2010). doi:10.1080/14772011003594870

172. P. R. Bell, T. Brougham, M. C. Herne, T. Frauenfelder, E. T. Smith, Fostoria dhimbangunmal, gen. et sp. nov., a new iguanodontian (Dinosauria, Ornithopoda) from the mid-Cretaceous of Lightning Ridge, New South Wales, Australia. J. Vertebr. Paleontol. 39, e1564757 (2019). doi:10.1080/02724634.2019.1564757

173. R. E. Molnar, S. W. Salisbury, in Thunder-Lizards: The Sauropodomorph Dinosaurs, V. Tidwell, K. Carpenter, Eds. (Indiana Univ. Press, 2005), pp. 454-465.

174. T. Brougham, E. T. Smith, P. R. Bell, Noasaurids are a component of the Australian 'mid'Cretaceous theropod fauna. Sci. Rep. 10, 1428 (2020). doi:10.1038/s41598-020-57667-7 Medline

175. T. Brougham, E. T. Smith, P. R. Bell, New theropod (Tetanurae: Avetheropoda) material from the 'mid'-Cretaceous Griman Creek Formation at Lightning Ridge, New South Wales, Australia [corrected]. R. Soc. Open Sci. 6, 180826 (2019). doi:10.1098/rsos.180826 Medline

176. H. Mallison, The digital Plateosaurus II: An assessment of the range of motion of the limbs and vertebral column and of previous reconstructions using a digital skeletal mount. Acta Palaeontol. Pol. 55, 433-458 (2010). doi:10.4202/app.2009.0075 
177. N. D. Smith, D. Pol, Anatomy of a basal sauropodomorph dinosaur from the Early Jurassic Hanson Formation of Antarctica. Acta Palaeontol. Pol. 52, 657-674 (2007).

178. Natural History Museum of Los Angeles County, “"Antarctic dinosaurs' makes its west coast debut at the Natural History Museum of Los Angeles County" (Natural History Museum of Los Angeles County, 2019); https://nhm.org/press/antarctic-dinosaurs.

179. W. R. Hammer, W. J. Hickerson, R. W. Slaughter, A dinosaur assemblage from the Transantarctic Mountains. Antarct. J. Rev. 29, 31-33 (1994).

180. C. L. Camp, A new type of small bipedal dinosaur from the Navajo Sandstone of Arizona. Univ. Calif. Publ. Geol. Sci. (1936).

181. N. D. Smith, P. J. Makovicky, D. Pol, W. R. Hammer, P. J. Currie, "The dinosaurs of the Early Jurassic Hanson Formation of the Central Transantarctic Mountains: Phylogenetic review and synthesis," in Antarctica: A Keystone in a Changing World-Online Proceedings of the 10th ISAES, A. K. Cooper, C. R. Raymond, 10th ISAES Editorial Team, Eds. (USGS Open-File Report 2007-1047, Short Research Paper 003, 2007); doi:10.3133/of2007-1047.srp003.

182. P. M. Galton, The species of the basal hypsilophodontid dinosaur Thescelosaurus Gilmore (Ornithischia: Ornithopoda) from the Late Cretaceous of North America. Neues Jahrb. für Geol. und Paläontologie - Abhandlungen 3, 297-311 (1995). doi:10.1127/njgpa/198/1995/297

183. M. B. Goodwin, New occurrences of pachycephalosaurid dinosaurs from the Hell Creek Formation, Garfield County, Montana. J. Vertebr. Paleontol. 3, 23A (1989).

184. D. J. Varricchio, in Mesozoic Vertebrate Life, D. H. Tanke, K. Carpenter, Eds. (Indiana Univ. Press, 2001), pp. 42-57.

185. G. F. Funston, P. J. Currie, M. E. Burns, New Elmisaurine specimens from North America and their relationship to the Mongolian Elmisaurus rarus. Acta Palaeontol. Pol. 61, 159173 (2016). doi:10.4202/app.00129.2014

186. T. E. Williamson, T. D. Carr, A new genus of derived pachycephalosaurian from western North America. J. Vertebr. Paleontol. 22, 779-801 (2003). doi:10.1671/02724634(2002)022[0779:ANGODP]2.0.CO;2

187. C. A. Boyd, C. M. Brown, R. D. Scheetz, J. A. Clarke, Taxonomic revison of the basal neornithischian taxa Thescelosaurus and Bugenasaura. J. Vertebr. Paleontol. 29, 758-770 (2009). doi:10.1671/039.029.0328

188. C. J. Ott, in Horns and Beaks: Ceratopsian and Ornithopod Dinosaurs, K. Carpenter, Ed. (Univ. of Indiana Press, 2006), pp. 213-234. doi:10.2307/j.ctt1zxz1md

189. T. W. Stanton, The age and strati graphic relations of the "Ceratops beds" of Wyoming and Montana. Wash. Acad. Sci. 11, 239-293 (1909); https://hdl.handle.net/10088/74447.

190. M. B. Goodwin, E. A. Buchholtz, R. E. Johnson, Cranial anatomy and diagnosis of Stygimoloch spinifer (Ornithischia: Pachycephalosauria) with comments on cranial display structures in agonistic behavior. J. Vertebr. Paleontol. 18, 363-375 (1998). doi:10.1080/02724634.1998.10011064 
191. K. Carpenter, Redescription of Ankylosaurus magniventris Brown 1908 (Ankylosauridae) from the Upper Cretaceous of the Western Interior of North America. Can. J. Earth Sci. 41, 961-986 (2004). doi:10.1139/e04-043

192. M. Wosik, M. B. Goodwin, D. C. Evans, A nestling-sized skeleton of Edmontosaurus (Ornithischia, Hadrosauridae) from the Hell Creek Formation of northeastern Montana, U.S.A., with an analysis of ontogenetic limb allometry. J. Vertebr. Paleontol. 37, e1398168 (2018). doi:10.1080/02724634.2017.1398168

193. A. T. McDonald, C. E. Campbell, B. Thomas, A new specimen of the controversial chasmosaurine Torosaurus latus (Dinosauria: Ceratopsidae) from the Upper Cretaceous hell creek formation of Montana. PLOS ONE 11, e0151453 (2016). doi:10.1371/journal.pone.0151453 Medline

194. D. A. Pearson, T. Schaefer, K. R. Johnson, D. J. Nichols, J. P. Hunter, in The Hell Creek Formation and the Cretaceous-Tertiary boundary in the northern Great Plains: An Integrated continental record of the end of the Cretaceous (Special Papers of the Geological Society of America, 2002), vol. 361. doi:10.1130/SPE361

195. J. H. Hartman, K. R. Johnson, D. J. Nichols, in The Hell Creek Formation and the Cretaceous-Tertiary boundary in the northern Great Plains: An Integrated continental record of the end of the Cretaceous (Special Papers of the Geological Society of America, 2002), vol. 361. doi:10.1130/SPE361.

196. J. T. Sankey, in Vertebrate Microfossil Assemblages: Their Role in Paleoecology and Paleobiogeography, J. T. Sankey, S. Baszio, Eds. (Indiana Univ. Press, 2008), pp. 123144.

197. L. E. Wilson, Comparative taphonomy and paleoecological reconstruction of two microvertebrate accumulations from the Late Cretaceous Hell Creek Formation (Maastrichtian), eastern Montana. Palaios 23, 289-297 (2008). doi:10.2110/palo.2007.p07-006r

198. T. A. Gates, L. E. Zanno, P. J. Makovicky, Theropod teeth from the upper Maastrichtian Hell Creek Formation "Sue" quarry: New morphotypes and faunal comparisons. Acta Palaeontol. Pol. 60, 131-139 (2013).

199. D. C. Evans, D. W. Larson, P. J. Currie, A new dromaeosaurid (Dinosauria: Theropoda) with Asian affinities from the latest Cretaceous of North America. Naturwissenschaften 100, 1041-1049 (2013). doi:10.1007/s00114-013-1107-5 Medline

200. M. Greenwald, thesis, South Dakota School of Mines and Technology, Rapid City, SD (1971).

201. M. C. Lamanna, H. D. Sues, E. R. Schachner, T. R. Lyson, A new large-bodied oviraptorosaurian theropod dinosaur from the latest Cretaceous of western North America. PLOS ONE 9, e92022 (2014). doi:10.1371/journal.pone.0092022 Medline

202. R. A. DePalma, D. A. Burnham, L. D. Martin, P. L. Larson, R. T. Bakker, The first giant raptor (Theropoda: Dromaeosauridae) from the Hell Creek Formation. Univ. Kansas Paleontol. Contrib. 14, 1-16 (2015).

203. D. A. Eberth, S. L. Kamo, High-precision U-PB CA-ID-TIMS dating and 
chronostratigraphy of the dinosaur-rich horseshoe canyon formation (Upper Cretaceous, Campanian-Maastrichtian), Red Deer River Valley, Alberta, Canada. Can. J. Earth Sci. 57, 1220-1237 (2020). doi:10.1139/cjes-2019-0019

204. W. Coombs, thesis, Columbia University, New York, NY (1971).

205. D. C. Evans, T. M. Cullen, D. W. Larson, A. Rego, A new species of troodontid theropod (Dinosauria: Maniraptora) from the Horseshoe Canyon formation (Maastrichtian) of Alberta, Canada. Can. J. Earth Sci. 54, 813-826 (2017). doi:10.1139/cjes-2017-0034

206. E. Chung, "Dinosaur's special arm muscles likely used to flex feathers in mating display." CBC News, 14 April 2016.

207. A. Sahni, The vertebrate fauna of the Judith River Formation, Montana. Bull. Am. Mus. Nat. Hist. 147, 6 (1972); http://hdl.handle.net/2246/1099.

208. J. C. Mallon, C. J. Ott, P. L. Larson, E. M. Iuliano, D. C. Evans, Spiclypeus shipporum gen. et sp. nov., a boldly audacious new chasmosaurine ceratopsid (Dinosauria: Ornithischia) from the Judith River Formation (Upper Cretaceous: Campanian) of Montana, USA. PLOS ONE 11, e0154218 (2016). doi:10.1371/journal.pone.0154218 Medline

209. M. J. Ryan, D. C. Evans, P. J. Currie, M. A. Loewen, A new chasmosaurine from northern Laramidia expands frill disparity in ceratopsid dinosaurs. Naturwissenschaften 101, 505512 (2014). doi:10.1007/s00114-014-1183-1 Medline

210. M. J. Ryan, in Geological Society of America Abstracts with Programs (Geological Society of America, 2006), p. 62.

211. V. M. Arbour, D. C. Evans, A new ankylosaurine dinosaur from the Judith River Formation of Montana, USA, based on an exceptional skeleton with soft tissue preservation. $R$. Soc. Open Sci. 4, 161086 (2017). doi:10.1098/rsos.161086 Medline

212. C. A. Boyd, S. K. Drumheller, T. A. Gates, Crocodyliform feeding traces on juvenile ornithischian dinosaurs from the Upper Cretaceous (Campanian) Kaiparowits Formation, Utah. PLOS ONE 8, e57605 (2013). doi:10.1371/journal.pone.0057605 Medline

213. L. E. Zanno, M. A. Loewen, A. A. Farke, G. S. Kim, L. P. A. M. Claessens, C. T. McGarrity, in At the Top of the Grand Staircase: The Late Cretaceous of Southern Utah (Univ. of Indiana Press, 2013), pp. 504-525.

214. J. P. Wiersma, R. B. Irmis, A new southern Laramidian ankylosaurid, Akainacephalus johnsoni gen. et sp. nov., from the upper Campanian Kaiparowits Formation of southern Utah, USA. PeerJ 6, e5016 (2018). doi:10.7717/peerj.5016 Medline

215. S. D. Sampson, E. K. Lund, M. A. Loewen, A. A. Farke, K. E. Clayton, A remarkable shortsnouted horned dinosaur from the Late Cretaceous (late Campanian) of southern Laramidia. Proc. Biol. Sci. 280, 20131186 (2013). doi:10.1098/rspb.2013.1186 Medline

216. S. D. Sampson, M. A. Loewen, A. A. Farke, E. M. Roberts, C. A. Forster, J. A. Smith, A. L. Titus, New horned dinosaurs from Utah provide evidence for intracontinental dinosaur endemism. PLOS ONE 5, e12292 (2010). doi:10.1371/journal.pone.0012292 Medline

217. T. A. Gates, S. D. Sampson, A new species of Gryposaurus (Dinosauria: Hadrosauridae) from the late Campanian Kaiparowits Formation, southern Utah, USA. Zool. J. Linn. Soc. 
151, 351-376 (2007). doi:10.1111/j.1096-3642.2007.00349.x

218. T. E. Williamson, R. M. Sullivan, Reinterpretation of a Parasaurolophus skull from the Upper Cretaceous (Campanian) Kaiparowits Formation, Utah. J. Vertebr. Paleontol. 17, 84A (1997).

219. L. E. Zanno, D. J. Varricchio, P. M. O’Connor, A. L. Titus, M. J. Knell, A new troodontid theropod, Talos sampsoni gen. et sp. nov., from the Upper Cretaceous Western Interior Basin of North America. PLOS ONE 6, e24487 (2011). doi:10.1371/journal.pone.0024487 Medline

220. L. E. Zanno, S. D. Sampson, A new oviraptorosaur (Theropoda, Maniraptora) from the Late Cretaceous (Campanian) of Utah. J. Vertebr. Paleontol. 25, 897-904 (2005). doi:10.1671/0272-4634(2005)025[0897:ANOTMF]2.0.CO;2

221. T. D. Carr, T. E. Williamson, B. B. Britt, K. Stadtman, Evidence for high taxonomic and morphologic tyrannosauroid diversity in the Late Cretaceous (late Campanian) of the American Southwest and a new short-skulled tyrannosaurid from the Kaiparowits Formation of Utah. Naturwissenschaften 98, 241-246 (2011). doi:10.1007/s00114-011$\underline{0762-7} \underline{\text { Medline }}$

222. R. S. Tykoski, Vertebrate paleontology in the Arizona Jurassic. Mesa Southwest Museum Bull. 11, 72-93 (2005).

223. J. M. Clark, D. E. Fastovsky, in The Beginning of the Age of the Dinosaurs: Faunal Change Across the Triassic-Jurassic Boundary, K. Padian, Ed. (Cambridge Univ. Press, 1986), pp. 285-302.

224. J. J. W. Sertich, M. A. Loewen, A new basal sauropodomorph dinosaur from the lower Jurassic Navajo sandstone of southern Utah. PLOS ONE 5, e9789 (2010). doi:10.1371/journal.pone.0009789 Medline

225. T. B. Rowe, H. D. Sues, R. R. Reisz, Dispersal and diversity in the earliest North American sauropodomorph dinosaurs, with a description of a new taxon. Proc. Biol. Sci. 278, 1044-1053 (2010). doi:10.1098/rspb.2010.1867 Medline

226. K. Padian, Presence of the dinosaur Scelidosaurus indicates Jurassic age for the Kayenta Formation (Glen Canyon Group, northern Arizona). Geology 17, 438 (1989). doi:10.1130/0091-7613(1989)017<0438:POTDSI>2.3.CO;2

227. R. Gay, in Notes on Early Mesozoic Theropods (Lulu Press, 2010), pp. 1-44.

228. T. Rowe, A new species of the theropod dinosaur Syntarsus from the early Jurassic Kayenta Formation of Arizona. J. Vertebr. Paleontol. 9, 125-136 (1989). doi:10.1080/02724634.1989.10011748

229. R. Gay, in The Carnivorous Dinosaurs (Life of the Past), K. Carpenter, Ed. (Indiana Univ. Press, 2005), pp. 277-283.

230. A. Averianov, P. Skutschas, A eutherian mammal from the early cretaceous of Russia and biostratigraphy of the Asian early Cretaceous vertebrate assemblages. Lethaia 33, 330340 (2007). doi:10.1080/002411600750053899

231. T. A. Gates, K. Tsogtbaatar, L. E. Zanno, T. Chinzorig, M. Watabe, A new iguanodontian 
(Dinosauria: Ornithopoda) from the Early Cretaceous of Mongolia. PeerJ 6, e5300 (2018). doi:10.7717/peerj.5300 Medline

232. A. O. Averianov, H. D. Sues, A new Troodontid (Dinosauria: Theropoda) from the Cenomanian of Uzbekistan, with a review of Troodontid records from the territories of the former Soviet Union. J. Vertebr. Paleontol. 27, 87-98 (2007). doi:10.1671/02724634(2007)27[87:ANTDTF]2.0.CO;2

233. R. M. Sullivan, S. G. Lucas, D. R. Braman, in New Mexico Geological Society 56th Field Conference Guidebook, S. G. Lucas, K. E. Zeigler, V. W. Lueth, D. E. Owen, Eds. (New Mexico Geological Society, 2005), pp. 395-407.

234. R. M. Sullivan, in New Mexico Geological Society 48th Field Conference Guidebook, O. Anderson, B. S. Kues, S. G. Lucas, Eds. (New Mexico Geological Society, 1997), pp. 249-253.

235. V. M. Arbour, M. E. Burns, R. M. Sullivan, S. G. Lucas, A. K. Cantrell, J. Fry, T. L. Suazo, A new ankylosaurid dinosaur from the Upper Cretaceous (Kirtlandian) of New Mexico with implications for ankylosaurid diversity in the Upper Cretaceous of western North America. PLOS ONE 9, e108804 (2014). doi:10.1371/journal.pone.0108804 Medline

236. N. R. Longrich, Titanoceratops ouranos, a giant horned dinosaur from the late Campanian of New Mexico. Cretac. Res. 32, 264-276 (2011). doi:10.1016/j.cretres.2010.12.007

237. D. W. Fowler, R. M. Sullivan, The first giant titanosaurian sauropod from the upper Cretaceous of North America. Acta Palaeontol. Pol. 56, 685-690 (2011). doi:10.4202/app.2010.0105

238. S. G. Sullivan, R. M. Lucas, in Fossil Vertebrates in New Mexico (Bulletin 68, New Mexico Museum of Natural History and Science, 2015).

239. T. E. Williamson, S. L. Brusatte, Small theropod teeth from the Late Cretaceous of the San Juan Basin, northwestern New Mexico and their implications for understanding latest Cretaceous dinosaur evolution. PLOS ONE 9, e93190 (2014).

doi:10.1371/journal.pone.0093190 Medline

240. D. C. Evans, D. W. Larson, T. M. Cullen, R. M. Sullivan, "Saurornitholestes" robustus is a troodontid (Dinosauria: Theropoda). Can. J. Earth Sci. 51, 730-734 (2014). doi:10.1139/cjes-2014-0073

241. T. D. Carr, T. E. Williamson, Bistahieversor sealeyi, gen. et sp. nov., a new tyrannosauroid from New Mexico and the origin of deep snouts in Tyrannosauroidea. J. Vertebr.

Paleontol. 30, 1-16 (2010). doi:10.1080/02724630903413032

242. P. M. Galton, Earliest record of an ankylosaurian dinosaur (Ornithischia: Thyreophora): Dermal armor from Lower Kota Formation (Lower Jurassic) of India. Neues Jahrb. für Geol. und Paläontologie-Abhandlungen 291, 205-219 (2019). doi:10.1127/njgpa/2019/0800

243. R. E. Ulanksy, "Evolution of the stegosaurs (Dinosauria; Ornithischia)," in Dinologia (2014), pp. 1-35; http://dinoweb.narod.ru/.

244. P. Yadagiri, A new sauropod Kotasaurus yamanpalliensis from Lower Jurassic Kota Formation of India. Rec. Geol. Surv. India 11, 102-127 (1988). 
245. S. L. Jain, T. S. Kutty, T. Roy-Chowdhury, S. Chatterjee, The sauropod dinosaur from the Lower Jurassic Kota Formation of India. Proc. R. Soc. London Ser. A 188, 221-228 (1975).

246. J. A. Wilson, P. C. Sereno, S. Srivastava, D. K. Bhatt, A. Khosla, A. Sahni, “A new Abelisaurid (Dinosauria, Theropoda) from the Lameta Formation (Cretaceous, Maastrichtian) of India: Contributions from the Museum of Paleontology" (Univ. of Michigan, 2003); https://d3qi0qp55mx5f5.cloudfront.net/paulsereno/i/docs/03-UMRajasaurus.pdf.

247. J. A. Wilson, M. D. D’Emic, K. Curry Rogers, D. M. Mohabey, S. Sen, "Reassessment of the Sauropod Dinosaur Jainosaurus (=“Antarctosaurus") septentrionalis from the Upper Cretaceous of India: Contributions from the Museum of Paleontology" (Univ. of Michigan, 2009); https://deepblue.lib.umich.edu/bitstream/handle/2027.42/63584/Contributions_32_no2_F INAL_07-14-09.pdf; sequence=1.

248. R. S. Loyal, D. M. Mohabey, A. Khosla, A. Sahni, Status and palaeobiology of the Late Cretaceous Indian theropods with description of a new theropod eggshell oogenus and oospecies, Ellipsoolithus khedaensis, from the Lameta Formation, District Kheda, Gujarat, Western India. Gaia 15, 379-387 (1998).

249. D. M. Mohabey, B. Samant, Cretaceous-Paleogene transition of reptilian tetrapods across deccan volcanism in India. Open J. Geol. 09, 639-642 (2019). doi:10.4236/ojg.2019.910062

250. D. Lambert, The Wordsworth Book of Dinosaurs (Wordsworth, 1998).

251. G. S. Paul, Predatory Dinosaurs of the World, A Complete Illustrated Guide (Simon \& Schuster, 1989).

252. F. E. Novas, S. Chatterjee, D. K. Rudra, P. M. Datta, in New Aspects of Mesozoic Biodiversity, S. Bandopadhyay, Ed. (Springer, 2010); pp. 45-62.

253. J. H. Ostrom, Leptoceratops gracilis from the "Lance" formation of Wyoming. J. Paleontol. 52, 697-704 (1978).

254. B. Brown, E. M. Schlaikjer, A study of the troodont dinosaurs with the description of a new genus and four new species. Bull. Am. Mus. Nat. Hist. 82, 115-150 (1943).

255. O. C. Marsh, Notice of new reptiles from the Laramie Formation. Am. J. Sci. s3-43, 449453 (1892). doi:10.2475/ajs.s3-43.257.449

256. K. L. Derstler, The Dragons' Grave: An Edmontosaurus bonebed containing theropod egg shells and juveniles, Lance Formation (uppermost Cretaceous), Niobrara County, Wyoming. J. Vertebr. Paleontol. 15, 26A (1995).

257. E. Tschopp, O. Mateus, R. B. J. Benson, A specimen-level phylogenetic analysis and taxonomic revision of Diplodocidae (Dinosauria, Sauropoda). PeerJ 3, e857 (2015). doi: $10.7717 /$ peerj.857 Medline

258. K. Snyder, M. A. McLain, I. Snyder, A. V. Chadwick, paper presented at the Geological Society of America, Southeastern Section 67th Annual Meeting, Indianapolis, IN, USA, 4-7 November 2018. 
259. S. G. Dalman, New examples of Tyrannosaurus rex from the Lance Formation of Wyoming, United States. Bull. Peabody Mus. Nat. Hist. 54, 241-254 (2013). doi:10.3374/014.054.0202

260. K. Carpenter, Late Cretaceous dinosaurs from the Denver Basin, Colorado. Rocky Mt. Geol. 37, 237-254 (2002). doi:10.2113/11

261. J. B. Hatcher, O. C. Marsh, R. S. Lull, “The Ceratopsia” (Monograph 49, U. S. Geological Survey, 1907).

262. M. T. Antunes, O. Mateus, Dinosaurs of Portugal. C. R. Palevol 2, 77-95 (2003). doi:10.1016/S1631-0683(03)00003-4

263. O. Mateus, S. C. R. Maidment, N. A. Christiansen, A new long-necked 'sauropod-mimic' stegosaur and the evolution of the plated dinosaurs. Proc. Biol. Sci. 276, 1815-1821 (2009). doi:10.1098/rspb.2008.1909 Medline

264. C. Hendrickx, O. Mateus, Abelisauridae (Dinosauria: Theropoda) from the Late Jurassic of Portugal and dentition-based phylogeny as a contribution for the identification of isolated theropod teeth. Zootaxa 3759, 1-74 (2014). doi:10.11646/zootaxa.3759.1.1 Medline

265. L. Mao, L. Xing, J. Zhang, T. Wang, D. Wang, Revisiting the world famous Lufeng Formation dinosaur fauna: New approaches to old problems. Hist. Biol. 32, 1062-1070 (2019). doi:10.1080/08912963.2018.1563784

266. H. L. You, Y. Azuma, T. Wang, Y. M. Wang, Z. M. Dong, The first well-preserved coelophysoid theropod dinosaur from Asia. Zootaxa 3873, 233-249 (2014). doi:10.11646/zootaxa.3873.3.3 Medline

267. K. C. Rogers, C. A. Forster, The skull of Rapetosaurus Krausei (Sauropoda: Titanosauria) from the Late Cretaceous of Madagascar. J. Vertebr. Paleontol. 24, 121-144 (2004). doi:10.1671/A1109-10

268. K. C. Rogers, J. A. Wilson, Vahiny depereti, gen. et sp. nov., a new titanosaur (Dinosauria, Sauropoda) from the Upper Cretaceous Maevarano Formation, Madagascar. J. Vertebr. Paleontol. 34, 606-617 (2014). doi:10.1080/02724634.2013.822874

269. P. M. O'Connor, C. A. Forster, A late Cretaceous (Maastrichtian) Avifauna from the Maevarano Formation, Madagascar. J. Vertebr. Paleontol. 30, 1178-1201 (2010). doi:10.1080/02724634.2010.483544

270. D. C. Evans, R. K. Schott, D. W. Larson, C. M. Brown, M. J. Ryan, The oldest North American pachycephalosaurid and the hidden diversity of small-bodied ornithischian dinosaurs. Nat. Commun. 4, 1828 (2013). doi:10.1038/ncomms2749 Medline

271. M. J. Ryan, D. C. Evans, P. J. Currie, C. M. Brown, D. Brinkman, New leptoceratopsids from the Upper Cretaceous of Alberta, Canada. Cretac. Res. 35, 69-80 (2012). doi:10.1016/j.cretres.2011.11.018

272. D. W. Larson, thesis, University of Alberta, Edmonton, Alberta, Canada (2010).

273. C. E. Turner, F. Peterson, in Vertebrate Palaeontology in Utah, Issue 1, D. D. Gillette, Ed. (Utah Geological Survey, 1999).

274. P. M. Sander, A. Christian, M. Clauss, R. Fechner, C. T. Gee, E. M. Griebeler, H. C. 
Gunga, J. Hummel, H. Mallison, S. F. Perry, H. Preuschoft, O. W. M. Rauhut, K. Remes, T. Tütken, O. Wings, U. Witzel, Biology of the sauropod dinosaurs: The evolution of gigantism. Biol. Rev. Camb. Philos. Soc. 86, 117-155 (2010). doi:10.1111/j.1469185X.2010.00137.x Medline

275. J. Foster, Jurassic West: The Dinosaurs of the Morrison Formation and Their World (Indiana Univ. Press, 2007).

276. D. D. Gillette, Seismosaurus Halli, gen. et sp. nov., a new sauropod dinosaur from the morrison formation (upper jurassic/lower cretaceous) of New Mexico, USA. J. Vertebr. Paleontol. 11, 417-433 (1991). doi:10.1080/02724634.1991.10011413

277. F. Therrien, D. M. Henderson, My theropod is bigger than yours...or not: Estimating body size from skull length in theropods. J. Vertebr. Paleontol. 27, 108-115 (2007). doi:10.1671/0272-4634(2007)27[108:MTIBTY]2.0.CO;2

278. D. J. Chure, M. A. Loewen, Cranial anatomy of Allosaurus jimmadseni, a new species from the lower part of the Morrison Formation (Upper Jurassic) of Western North America. PeerJ 8, e7803 (2020). doi:10.7717/peerj.7803 Medline

279. E. L. Nicholls, A. P. Russell, New specimen of Struthiomimus altus from Alberta, with comments on the classificatory characters of Upper Cretaceous ornithomimids. Can. J. Earth Sci. 18, 518-526 (1981). doi:10.1139/e81-045

280. D. C. Evans, M. J. Ryan, Cranial anatomy of Wendiceratops pinhornensis gen. et sp. nov., a centrosaurine ceratopsid (Dinosauria: Ornithischia) from the Oldman Formation (Campanian), Alberta, Canada, and the evolution of ceratopsid nasal ornamentation. PLOS ONE 10, e0130007 (2015). doi:10.1371/journal.pone.0130007 Medline

281. A. Boyle, "Wendiceratops: Horned dinosaur fossil adds hooks to evolutionary tale," NBC News, 8 July 2015.

282. K. Carpenter, Skeletal and dermal armor reconstruction of Euoplocephalus tutus (Ornithischia: Ankylosauridae) from the Late Cretaceous Oldman Formation of Alberta. Can. J. Earth Sci. 19, 689-697 (1982). doi:10.1139/e82-058

283. A. A. Farke, M. J. Ryan, P. M. Barrett, D. H. Tanke, D. R. Braman, M. A. Loewen, M. R. Graham, A new centrosaurine from the Late Cretaceous of Alberta, Canada, and the evolution of parietal ornamentation in horned dinosaurs. Acta Palaeontol. Pol. 56, 691702 (2011). doi:10.4202/app.2010.0121

284. K. Chiba, M. J. Ryan, D. R. Braman, D. A. Eberth, E. E. Scott, C. M. Brown, Y. Kobayashi, D. C. Evans, Taphonomy of a monodominant Centrosaurus apertus (Dinosauria: Ceratopsia) bonebed from the Upper Oldman Formation of Southeastern Alberta. Palaios 30, 655-667 (2015). doi:10.2110/palo.2014.084

285. M. J. Ryan, A new basal centrosaurine ceratopsid from the Oldman Formation, southeastern Alberta. J. Paleontol. 81, 376-396 (2007).

286. J. Peng, D. Brinkman, A. P. Russell, "Vertebrate microsite assemblages (exclusive of mammals) from the Foremost and Oldman formations of the Judith River Group (Campanian) of southeastern Alberta: An illustrated guide" (Occasional Paper 25, Provinical Museum of Alberta, 2001). 
287. C. M. Brown, P. Druckenmiller, Basal ornithopod (Dinosauria: Ornithischia) teeth from the Prince Creek Formation (early Maastrichtian) of Alaska. Can. J. Earth Sci. 48, 13421354 (2011). doi:10.1139/e11-017

288. J. F. Anderson, A. Hall-Martin, D. A. Russell, Long-bone circumference and weight in mammals, birds and dinosaurs. J. Zool. 207, 53-61 (2009). doi:10.1111/j.14697998.1985.tb04915.x

289. R. A. Gangloff, A. R. Fiorillo, D. W. Norton, The first pachycephalosaurine (Dinosauria) from the Paleo-Arctic of Alaska and its paleogeographic implications. J. Paleontol. 79, 997-1001 (2005). doi:10.1666/0022-3360(2005)079[0997:TFPDFT]2.0.CO;2

290. J. M. Parrish, J. T. Parrish, J. H. Hutchison, R. A. Spicer, Late Cretaceous vertebrate fossils from the North Slope of Alaska and implications for dinosaur ecology. Palaios 2, 377 (1987). doi:10.2307/3514763

291. R. S. Tykoski, A. R. Fiorillo, K. Chiba, New data and diagnosis for the Arctic ceratopsid dinosaur Pachyrhinosaurus perotorum. J. Syst. Palaeontology 17, 1397-1416 (2019). doi:10.1080/14772019.2018.1532464

292. R. Takasaki, A. R. Fiorillo, Y. Kobayashi, R. S. Tykoski, P. J. McCarthy, The first definite lambeosaurine bone from the Liscomb bonebed of the Upper Cretaceous Prince Creek Formation, Alaska, United States. Sci. Rep. 9, 5384 (2019). doi:10.1038/s41598-019$\underline{41325-8} \underline{\text { Medline }}$

293. A. R. Fiorillo, Microwear patterns on the teeth of northern high latitude hadrosaurs with comments on microwear patterns in hadrosaurs as a function of latitude and seasonal ecological constraints. Palaeontol. Electron. 14, PE14.3.20A (2011); https://palaeoelectronica.org/2011_3/7_fiorillo/7_fiorillo.pdf.

294. A. R. Fiorillo, R. S. Tykoski, A diminutive new tyrannosaur from the top of the world. PLOS ONE 9, e91287 (2014). doi:10.1371/journal.pone.0091287 Medline

295. K. Li, C. Yang, F. Hu, Dinosaur assemblages from the Middle Jurassic Shaximiao Formation and Chuanjie Formation in the Sichuan-Yunnan Basin, China. Vol. Jurassica 9, 21-42 (2011).

296. Z. Dong, On a small ornithopod (Gongbusaurus wucaiwanensis sp. nov.) from Kelamaili, Jungar Basin, Xinjiang, China. Vertebr. Palasiat. 27, 140-146 (1989).

297. Y. Zhang, W. Chen, in Transactions of the Continental Jurassic Symposium, Museum of Northern Arizona (Flagstaff, 1996), pp. 97-107.

298. Z.-M. Dong, S. Zhou, Y. Zhang, Dinosaurs from the Jurassic of Sichuan. Palaeontol. Sin. 162, 1-136 (1983).

299. S. L. Brusatte, R. B. J. Benson, X. Xu, The evolution of large-bodied theropod dinosaurs during the mesozoic in asia. J. Iber. Geol. 36, 275-296 (2010). doi:10.5209/rev JIGE.2010.v36.n2.12

300. O. Mateus, Late Jurassic dinosaurs from the Morrison Formation (USA), the Lourinhã and Alcobaça Formations (Portugal), and the Tendaguru beds (Tanzania): a comparison. New Mex. Museum Nat. Hist. Sci. Bull. 36, 223-231 (2006). 
301. D. Schwarz, J. C. D. Kosch, G. Fritsch, T. Hildebrandt, Dentition and tooth replacement of Dicraeosaurus hansemanni (Dinosauria, Sauropoda, Diplodocoidea) from the Tendaguru Formation of Tanzania. J. Vertebr. Paleontol. 35, e1008134 (2015). doi:10.1080/02724634.2015.1008134

302. M. P. Taylor, A re-evaluation of Brachiosaurus altithorax Riggs 1903 (Dinosauria, Sauropoda) and its generic separation from Giraffatitan brancai (Janensch 1914). $J$. Vertebr. Paleontol. 29, 787-806 (2009). doi:10.1671/039.029.0309

303. O. W. M. Rauhut, Theropod dinosaurs from the Late Jurassic of Tendaguru (Tanzania). Spec. Pap. Palaeontol. 86, 195-239 (2011).

304. O. W. M. Rauhut, Post-cranial remains of "coelurosaurs" (Dinosauria, Theropoda) from the Late Jurassic of Tanzania. Geol. Mag. 142, 97-107 (2005). doi: $10.1017 /$ S0016756804000330

305. K. Remes, Taxonomy of Late Jurassic diplodocid sauropods from Tendaguru (Tanzania). Foss. Rec. 12, 23-46 (2009). doi:10.1002/mmng.200800008

306. P. D. Mannion, P. Upchurch, D. Schwarz, O. Wings, Taxonomic affinities of the putative titanosaurs from the Late Jurassic Tendaguru Formation of Tanzania: Phylogenetic and biogeographic implications for eusauropod dinosaur evolution. Zool. J. Linn. Soc. 185, 784-909 (2019). doi:10.1093/zoolinnean/zly068

307. E. Buffetaut, An early spinosaurid dinosaur from the Late Jurassic of Tendaguru (Tanzania) and the evolution of the spinosaurid dentition. Oryctos 10, 1-8 (2012).

308. M. L. Casanovas, X. Pereda Suberbiola, J. V. Santafé, D. B. Weishampel, A primitive euhadrosaurian dinosaur from the uppermost Cretaceous of the Ager syncline (southern Pyrenees, Catalonia). Geol. en Mijnbouw/Netherlands. J. Geosci. 78, 345-356 (1999).

309. V. Riera, O. Oms, R. Gaete, À. Galobart, The end-Cretaceous dinosaur succession in Europe: The Tremp Basin record (Spain). Palaeogeogr. Palaeoclimatol. Palaeoecol. 283, 160-171 (2009). doi:10.1016/j.palaeo.2009.09.018

310. H. K. Erben, J. Hoefs, K. H. Wedepohl, Paleobiological and isotopic studies of eggshells from a declining dinosaur species. Paleobiology 5, 380-414 (2016). doi:10.1017/S0094837300016900

311. A. Torices, P. J. Currie, J. I. Canudo, X. Pereda-Suberbiola, Theropod dinosaurs from the Upper Cretaceous of the South Pyrenees basin of Spain. Acta Palaeontol. Pol. 60, 611626 (2013).

312. R. Allain, P. Taquet, A new genus of Dromaeosauridae (Dinosauria, Theropoda) from the Upper Cretaceous of France. J. Vertebr. Paleontol. 20, 404-407 (2000). doi:10.1671/0272-4634(2000)020[0404:ANGODD]2.0.CO;2

313. J. Lü, L. Yi, S. L. Brusatte, L. Yang, H. Li, L. Chen, A new clade of Asian late Cretaceous long-snouted tyrannosaurids. Nat. Commun. 5, 3788 (2014). Medline

314. T. A. Gates, S. D. Sampson, L. E. Zanno, E. M. Roberts, J. G. Eaton, R. L. Nydam, J. H. Hutchison, J. A. Smith, M. A. Loewen, M. A. Getty, Biogeography of terrestrial and freshwater vertebrates from the late Cretaceous (Campanian) Western Interior of North America. Palaeogeogr. Palaeoclimatol. Palaeoecol. 291, 371-387 (2010). 
doi:10.1016/j.palaeo.2010.03.008

315. B. Chinnery, Description of Prenoceratops pieganensis gen. et sp. Nov. (Dinosauria: Neoceratopsia) from the two medicine formation of montana. J. Vertebr. Paleontol. 24, 572-590 (2004). doi:10.1671/0272-4634(2004)024[0572:DOPPGE]2.0.CO;2

316. R. E. Barrick, W. J. Showers, A. G. Fischer, Comparison of thermoregulation of four ornithischian dinosaurs and a varanid lizard from the cretaceous two medicine formation: Evidence from oxygen isotopes. Palaios 11, 295 (1996). doi:10.2307/3515240

317. B. J. Chinnery, J. R. Horner, A new neoceratopsian Dinosaur linking North American and Asian taxa. J. Vertebr. Paleontol. 27, 625-641 (2007). doi:10.1671/02724634(2007)27[625:ANNDLN]2.0.CO;2

318. S. D. Sampson, Two new horned dinosaurs from the Upper Cretaceous two medicine formation of montana; with a phylogenetic analysis of the centrosaurinae (Ornithischia: Ceratopsidae). J. Vertebr. Paleontol. 15, 743-760 (1995). doi:10.1080/02724634.1995.10011259

319. J. P. Wilson, M. J. Ryan, D. C. Evans, A new, transitional centrosaurine ceratopsid from the Upper Cretaceous Two Medicine Formation of Montana and the evolution of the 'Styracosaurus-line' dinosaurs. R. Soc. Open Sci. 7, 200284 (2020). doi:10.1098/rsos.200284 Medline

320. P. Penkalski, A new ankylosaurid from the Late Cretaceous Two Medicine Formation of Montana, USA. Acta Palaeontol. Pol. 59, 617-634 (2014).

321. A. T. McDonald, J. R. Horner, in New Perspectives on Horned Dinosaurs: The Royal Tyrrell Museum Ceratopsian Symposium, M. J. Ryan, B. J. Chinnery-Allgeier, D. A. Eberth, Eds. (Indiana Univ. Press, 2010), pp. 156-168.

322. T. A. Gates, J. R. Horner, R. R. Hanna, C. R. Nelson, New unadorned hadrosaurine hadrosaurid (Dinosauria, Ornithopoda) from the Campanian of North America. $J$. Vertebr. Paleontol. 31, 798-811 (2011). doi:10.1080/02724634.2011.577854

323. D. J. Varricchio, Taphonomy of Jack's Birthday Site, a diverse dinosaur bonebed from the Upper Cretaceous Two Medicine Formation of Montana. Palaeogeogr. Palaeoclimatol. Palaeoecol. 114, 297-323 (1995). doi:10.1016/0031-0182(94)00084-L

324. D. A. Burnham, K. L. Derstler, P. J. Currie, R. T. Bakker, Z. Zhou, J. H. Ostrom, "Remarkable new birdlike dinosaur (Theropoda: Maniraptora) from the Upper Cretaceous of Montana" (Paleontological Contributions no. 13, University of Kansas, 2000).

325. N. R. Longrich, K. Barnes, S. Clark, L. Millar, Caenagnathidae from the upper Campanian Aguja Formation of west Texas, and a revision of the Caenagnathinae. Bull. Peabody Mus. Nat. Hist. 54, 23-49 (2013). doi:10.3374/014.054.0102

326. P. J. Currie, D. Trexler, E. B. Koppelhus, K. Wicks, N. Murphy, in The Carnivorous Dinosaurs, K. Carpenter, Ed. (Indiana Univ. Press, 2005), pp. 313-324.

327. R. J. Butler, The "fabrosaurid" ornithischian dinosaurs of the Upper Elliot Formation (Lower Jurassic) of South Africa and Lesotho. Zool. J. Linn. Soc. 145, 175-218 (2005). doi:10.1111/j.1096-3642.2005.00182.x 
328. J. A. Hopson, On the generic separation of the ornithischian dinosaurs Lycorhinus and Heterodontosaurus from the Stromberg Series (Upper Triassic) of South Africa. S. Afr. J. Sci. 71, 302-305 (1975).

329. P. C. Sereno, Taxonomy, morphology, masticatory function and phylogeny of heterodontosaurid dinosaurs. ZooKeys 226, 1-225 (2012). doi:10.3897/zookeys.226.2840 Medline

330. B. W. McPhee, E. M. Bordy, L. Sciscio, J. N. Choiniere, The sauropodomorph biostratigraphy of the elliot formation of southern Africa: Tracking the evolution of sauropodomorpha across the triassic-jurassic boundary. Acta Palaeontol. Pol. 62, (2017). doi:10.4202/app.00377.2017

331. A. M. Yates, M. F. Bonnan, J. Neveling, A new basal sauropodomorph dinosaur from the Early Jurassic of South Africa. J. Vertebr. Paleontol. 31, 610-625 (2011). doi: $10.1080 / 02724634.2011 .560626$

332. P. M. Barrett, A new basal sauropodomorph dinosaur from the Upper Elliot Formation (Lower Jurassic) of South Africa. J. Vertebr. Paleontol. 29, 1032-1045 (2009). doi:10.1671/039.029.0401

333. C. Peyre de Fabrègues, R. Allain, New material and revision of Melanorosaurus thabanensis, a basal sauropodomorph from the Upper Triassic of Lesotho. PeerJ 4, e1639 (2016). Medline

334. A. M. Yates, M. F. Bonnan, J. Neveling, A. Chinsamy, M. G. Blackbeard, A new transitional sauropodomorph dinosaur from the Early Jurassic of South Africa and the evolution of sauropod feeding and quadrupedalism. Proc. Biol. Sci. 277, 787-794 (2009). doi:10.1098/rspb.2009.1440 Medline

335. B. W. McPhee, M. F. Bonnan, A. M. Yates, J. Neveling, J. N. Choiniere, A new basal sauropod from the pre-Toarcian Jurassic of South Africa: Evidence of niche-partitioning at the sauropodomorph-sauropod boundary? Sci. Rep. 5, 13224 (2015). doi:10.1038/srep13224 Medline

336. D. Munyikwa, M. A. Raath, Further material of the ceratosaurian dinosaur Syntarsus from the Elliot Formation (early Jurassic) of South Africa. Palaeontol. Afr. 35, 55-99 (1999).

337. A. M. Yates, A new theropod dinosaur from the Early Jurassic of South Africa and its implications for the early evolution of theropods. Palaeontol. Africana 41, 105-122 (2005).

338. W. T. Blows, A review of Lower and Middle Cretaceous dinosaurs of England. New Mex. Museum Nat. Hist. Sci. Bull. 14, 29-38 (1998).

339. R. Allain, R. Vullo, J. Le Loeuff, J. F. Tournepiche, European ornithomimosaurs (Dinosauria, Theropoda): an undetected record. Geol. Acta 12, 127-135 (2014).

340. P. M. Barrett, S. C. R. Maidment, in English Wealden Fossils, D. J. Batten, Ed. (The Palaeontological Association, 2011), pp. 391-406.

341. J. Ruiz-Omeñaca, J. Canudo Sanagustín, "Pleurocoelus” valdensis Lydekker, 1889 (Saurischia, sauropoda) en el Cretácico Inferior (Barremiense) de la Península Ibérica. Geogaceta (2005). 
342. D. B. Norman, On the history, osteology, and systematic position of the Wealden (Hastings group) dinosaur Hypselospinus fittoni (Iguanodontia: Styracosterna). Zool. J. Linn. Soc. 173, 92-189 (2014). doi:10.1111/zoj.12193

343. K. Brooks, Dinosaur quarries of Hastings. Hast. Dist. Geol. Soc. J. 17, 7-13 (2011).

344. D. F. Glut, Dinosaurs: The Encyclopedia (McFarland, 1997).

345. P. Upchurch, P. D. Mannion, M. P. Taylor, The anatomy and phylogenetic relationships of "Pelorosaurus" becklesii (Neosauropoda, Macronaria) from the Early Cretaceous of England. PLOS ONE 10, e0125819 (2015). doi:10.1371/journal.pone.0125819 Medline

346. M. P. Taylor, Xenoposeidon is the earliest known rebbachisaurid sauropod dinosaur. PeerJ 6, e5212 (2018). doi:10.7717/peerj.5212 Medline

347. M. P. Taylor, D. Naish, An unusual new neosauropod dinosaur from the Lower Cretaceous Hastings Beds Group of East Sussex, England. Palaeontology 50, 1547-1564 (2007). doi:10.1111/j.1475-4983.2007.00728.x

348. P. Upchurch, J. Martin, The anatomy and taxonomy of Cetiosaurus (Saurischia, Sauropoda) from the Middle Jurassic of England. J. Vertebr. Paleontol. 23, 208-231 (2003). doi:10.1671/0272-4634(2003)23[208:TAATOC]2.0.CO;2

349. P. D. Mannion, A revision of the sauropod dinosaur genus "Bothriospondylus" with a redescription of the type material of the Middle Jurassic form "B. madagascariensis". Palaeontology 53, 277-296 (2010). doi:10.1111/j.1475-4983.2009.00919.x

350. J. W. Hulke, Note (3rd) on (Ecumerotus, Hulke) ornuithopsis, H. G. Seeley, = Bothriospondylus magnus, owen, = Chondrosteosaurus Magnus, Owen. Q. J. Geol. Soc. Lond. 35, 752-762 (1879). doi:10.1144/GSL.JGS.1879.035.01-04.55

351. D. Naish, S. C. Sweetman, A tiny maniraptoran dinosaur in the Lower Cretaceous Hastings Group: Evidence from a new vertebrate-bearing locality in south-east England. Cretac. Res. 32, 464-471 (2011). doi:10.1016/j.cretres.2011.03.001

352. P. Austen, D. Brockhurst, K. Honeysett, Vertebrate fauna from Ashdown brickworks, Bexhill, East Sussex. Wealden News 8, 13-23 (2010).

353. M. W. Maisch, The nomenclatural status of the carnivorous dinosaur genus Altispinax v. HUENE, 1923 (Saurischia, Theropoda) from the Lower Cretaceous of England. Neues Jahrb. Geol. Paläontol. Abh. 280, 215-219 (2016). doi:10.1127/njgpa/2016/0576

354. J. I. Kirkland, D. Deblieux, in New Perspectives on Horned Dinosaurs: The Royal Tyrell Museum Ceratopsian Symposium, M. J. Ryan, B. J. Chinnery-Allgeier, D. A. Eberth, Eds. (Indiana Univ. Press, 2010), pp. 117-140.

355. J. G. Eaton, R. L. Cifelli, J. H. Hutchison, J. I. Kirkland, J. M. Parrish, in Vertebrate Paleontology in Utah (Utah Geological Survey, Misc. Pub. 99-1, 1999), pp. 345-353.

356. J. G. Eaton, H. Munk, M. A. Hardman, in National Park Paleontological Research, V. L. Santucci, L. McClelland, Eds. (Tech. Rep. NPS/NRGRD/GRDTR-98/01, National Park Service, 1998); pp. 36-40.

357. T. A. Gates, Z. Jinnah, C. Levitt, M. A. Getty, in Hadrosaurs: Life of the Past, D. A. Eberth, D. C. Evans, Eds. (Indiana Univ. Press, 2014); pp. 361-384. 
358. A. L. Titus, J. G. Eaton, J. Sertich, Late Cretaceous stratigraphy and vertebrate faunas of the Markagunt, Paunsaugunt, and Kaiparowits plateaus, southern Utah. Geol. Intermt. West 3, 229-291 (2016). doi:10.31711/giw.v3.pp229-291

359. E. K. Lund, P. M. O’Connor, M. A. Loewen, Z. A. Jinnah, A New Centrosaurine Ceratopsid, Machairoceratops cronusi gen et sp. nov., from the Upper Sand Member of the Wahweap Formation (Middle Campanian), Southern Utah. PLOS ONE 11, e0154403 (2016). doi:10.1371/journal.pone.0154403 Medline

360. M. A. Loewen, R. B. Irmis, J. J. W. Sertich, P. J. Currie, S. D. Sampson, Tyrant dinosaur evolution tracks the rise and fall of Late Cretaceous oceans. PLOS ONE 8, e79420 (2013). doi:10.1371/journal.pone.0079420 Medline

361. G. Z. Peng, C. Shu, in Proceedings of the Seventh Annual Meeting of the Chinese Society of Vertebrate Paleontology (China Ocean Press, Beijing, 1999), pp. 27-35.

362. G. Z. Peng, Y. Ye, Y. Gao, C. Shu, S. Jiang, in Jurassic Dinosaur Faunas in Zigong, G. Z. Peng, Ed. (Sichuan People's Publishing House, 2005), pp. 81-85.

363. C. Tan, M. Xiao, H. Dai, X. F. Hu, N. Li, Q. Y. Ma, Z. Y. Wei, H. D. Yu, C. Xiong, G. Z. Peng, S. Jiang, X. X. Ren, H. L. You, A new species of Omeisaurus (Dinosauria: Sauropoda) from the Middle Jurassic of Yunyang, Chongqing, China. Hist. Biol. 32, 10.1080/08912963.2020.1743286 (2020). doi:10.1080/08912963.2020.1743286

364. Y. Gao, A new species of Szechuanosaurus from the Middle Jurassic of Dashanpu, Zigong, Sichuan. Vertebr. Palasiat. 31, 308-314 (1993).

365. P. M. Barrett, R. J. Butler, F. Knoll, Small-bodied ornithischian dinosaurs from the middle jurassic of sichuan, China. J. Vertebr. Paleontol. 25, 823-834 (2005). doi:10.1671/02724634(2005)025[0823:SODFTM]2.0.CO;2

366. Q. Ji, J. C. Lü, X. F. Wei, X. R. Wang, A new oviraptorosaur from the Yixian Formation of Jianchang, Western Liaoning Province, China. Geol. Bull. China 31, 2102-2107 (2012).

367. X. Xu, M. A. Norell, Non-avian dinosaur fossils from the Lower Cretaceous Jehol Group of western Liaoning, China. Geol. J. 41, 419-437 (2006). doi:10.1002/gj.1044

368. X. Wang, H. You, Q. Meng, C. Gao, X. Cheng, J. Liu, Dongbeititan dongi, the first sauropod dinosaur from the Lower Cretaceous Jehol Group of western Liaoning Province, China. Acta Geol. Sin. 81, 911-916 (2007).

369. G. Han, L. M. Chiappe, S. A. Ji, M. Habib, A. H. Turner, A. Chinsamy, X. Liu, L. Han, A new raptorial dinosaur with exceptionally long feathering provides insights into dromaeosaurid flight performance. Nat. Commun. 5, 4382 (2014). doi:10.1038/ncomms5382 Medline

370. J. Lü, S. L. Brusatte, A large, short-armed, winged dromaeosaurid (Dinosauria: Theropoda) from the Early Cretaceous of China and its implications for feather evolution. Sci. Rep. 5, 11775 (2015). Medline

371. H. Pu, Y. Kobayashi, J. Lü, L. Xu, Y. Wu, H. Chang, J. Zhang, S. Jia, An unusual basal Therizinosaur dinosaur with an ornithischian dental arrangement from northeastern China. PLOS ONE 8, e63423 (2013). doi:10.1371/journal.pone.0063423 Medline 
372. L. Xing, P. R. Bell, W. S. Persons 4th, S. Ji, T. Miyashita, M. E. Burns, Q. Ji, P. J. Currie, Abdominal contents from two large early cretaceous compsognathids (Dinosauria: Theropoda) demonstrate feeding on confuciusornithids and dromaeosaurids. PLOS ONE 7, e44012 (2012). doi:10.1371/journal.pone.0044012 Medline

373. X. Xu, K. Wang, K. Zhang, Q. Ma, L. Xing, C. Sullivan, D. Hu, S. Cheng, S. Wang, A gigantic feathered dinosaur from the lower cretaceous of China. Nature 484, 92-95 (2012). doi:10.1038/nature10906 Medline

374. J. Liyong, C. Jun, P. Godefroit, in Bernissart Dinosaurs and Early Cretaceous Terrestrial Ecosystems, P. Godefroid, Ed. (Indiana Univ. Press, 2012), pp. 467-488.

375. C. F. Zhou, K. Q. Gao, R. C. Fox, S. H. Chen, A new species of Psittacosaurus (Dinosauria: Ceratopsia) from the Early Cretaceous Yixian Formation, Liaoning, China. Palaeoworld 15, 100-114 (2006). doi:10.1016/j.palwor.2005.11.001

376. C. Z. Shen, B. Zhao, C. L. Gao, J. C. Lü, M. Kundrát, A new troodontid dinosaur (Liaoningvenator curriei gen. et sp. nov.) from the Early Cretaceous Yixian Formation in Western Liaoning Province. Acta Geosci. Sin. 38, 359-371 (2017). doi:10.3975/cagsb.2017.03.06

377. C. Shen, J. Lü, C. Gao, M. Hoshino, K. Uesugi, M. Kundrát, Forearm bone histology of the small theropod Daliansaurus liaoningensis (Paraves: Troodontidae) from the Yixian Formation, Liaoning, China. Hist. Biol. 31, 253-261 (2017). doi:10.1080/08912963.2017.1360296

378. C. Shen, J. Lu, S. Liu, M. Kundrát, S. L. Brusatte, H. Gao, A new troodontid dinosaur from the Lower Cretaceous Yixian Formation of Liaoning Province, China. Acta Geol. Sin. 91, 763-780 (2017). doi:10.1111/1755-6724.13307 


\section{Materials Design Analysis Reporting (MDAR) Checklist for Authors}

The MDAR framework establishes a minimum set of requirements in transparent reporting applicable to studies in the life sciences (see Statement of Task: doi:10.31222/osf.io/9sm4x.). The MDAR checklist is a tool for authors, editors and others seeking to adopt the MDAR framework for transparent reporting in manuscripts and other outputs. Please refer to the MDAR Elaboration Document for additional context for the MDAR framework. 


\section{Materials}

\begin{tabular}{|l|l|l|}
\hline Antibodies & Yes (indicate where provided: page no/section/legend) & n/a \\
\hline $\begin{array}{l}\text { For commercial reagents, provide supplier } \\
\text { name, catalogue number and RRID, if available. }\end{array}$ & & $x$ \\
\hline
\end{tabular}

\begin{tabular}{|l|l|l|}
\hline Cell materials & Yes (indicate where provided: page no/section/legend) & n/a \\
\hline $\begin{array}{l}\text { Cell lines: Provide species information, strain. } \\
\text { Provide accession number in repository OR } \\
\text { supplier name, catalog number, clone number, } \\
\text { OR RRID }\end{array}$ & & $x$ \\
\hline $\begin{array}{l}\text { Primary cultures: Provide species, strain, sex of } \\
\text { origin, genetic modification status. }\end{array}$ & & \\
\hline
\end{tabular}

\begin{tabular}{|l|l|l|}
\hline Experimental animals & Yes (indicate where provided: page no/section/legend) & n/a \\
\hline $\begin{array}{l}\text { Laboratory animals: Provide species, strain, sex, age, } \\
\text { genetic modification status. Provide accession } \\
\text { number in repository OR supplier name, catalog } \\
\text { number, clone number, OR RRID }\end{array}$ & & $\mathrm{x}$ \\
\hline $\begin{array}{l}\text { Animal observed in or captured from the } \\
\text { field: Provide species, sex and age where } \\
\text { possible }\end{array}$ & & \\
\hline $\begin{array}{l}\text { Model organisms: Provide Accession number } \\
\text { in repository (where relevant) OR RRID }\end{array}$ & & $\mathrm{x}$ \\
\hline
\end{tabular}

\begin{tabular}{|l|l|l|}
\hline Plants and microbes & Yes (indicate where provided: page no/section/legend) & n/a \\
\hline $\begin{array}{l}\text { Plants: provide species and strain, unique accession } \\
\text { number if available, and source (including location } \\
\text { for collected wild specimens) }\end{array}$ & & $\mathrm{x}$ \\
\hline $\begin{array}{l}\text { Microbes: provide species and strain, unique } \\
\text { accession number if available, and source }\end{array}$ & & $\mathrm{x}$ \\
\hline
\end{tabular}

\begin{tabular}{|l|l|l|}
\hline Human research participants & Yes (indicate where provided: page no/section/legend) & $\mathrm{n} / \mathrm{a}$ \\
\hline $\begin{array}{l}\text { Identify authority granting ethics approval (IRB or } \\
\text { equivalent committee(s), provide reference number } \\
\text { for approval. }\end{array}$ & & $\mathrm{x}$ \\
\hline $\begin{array}{l}\text { Provide statement confirming informed consent } \\
\text { obtained from study participants. }\end{array}$ & & $\mathrm{x}$ \\
\hline Report on age and sex for all study participants. & & $\mathrm{x}$ \\
\hline
\end{tabular}




\section{Design}

\begin{tabular}{|l|l|c|}
\hline Study protocol & Yes (indicate where provided: page no/section/legend) & n/a \\
\hline $\begin{array}{l}\text { For clinical trials, provide the trial registration } \\
\text { number OR cite DOI in manuscript. }\end{array}$ & & $x$ \\
\hline
\end{tabular}

\begin{tabular}{|l|c|c|}
\hline Laboratory protocol & Yes (indicate where provided: page no/section/legend) & n/a \\
\hline $\begin{array}{l}\text { Provide DOI or other citation details if detailed step- } \\
\text { by-step protocols are available. }\end{array}$ & & $x$ \\
\hline
\end{tabular}

\begin{tabular}{|l|l|c|}
\hline Experimental study design (statistics details) & Yes (indicate where provided: page no/section/legend) & n/a \\
\hline $\begin{array}{l}\text { State whether and how the following have been } \\
\text { done, or if they were not carried out. }\end{array}$ & & \\
\hline Sample size determination & Materials and Methods: lines 354-356 & \\
\hline Randomisation & Materials and Methods: line 379 & $\mathrm{X}$ \\
\hline Blinding & & \\
\hline Inclusion/exclusion criteria & Materials and Methods: lines 335-340 & \\
\hline
\end{tabular}

\begin{tabular}{|l|c|c|}
\hline Sample definition and in-laboratory replication & Yes (indicate where provided: page no/section/legend) & n/a \\
\hline $\begin{array}{l}\text { State number of times the experiment was } \\
\text { replicated in laboratory }\end{array}$ & $\mathrm{x}$ \\
\hline $\begin{array}{l}\text { Define whether data describe technical or biological } \\
\text { replicates }\end{array}$ & & $\mathrm{x}$ \\
\hline
\end{tabular}

\begin{tabular}{|l|c|c|}
\hline Ethics & Yes (indicate where provided: page no/section/legend) & n/a \\
\hline $\begin{array}{l}\text { Studies involving human participants: State details of } \\
\text { authority granting ethics approval (IRB or equivalent } \\
\text { committee(s), provide reference number for } \\
\text { approval. }\end{array}$ & & $\mathrm{x}$ \\
\hline $\begin{array}{l}\text { Studies involving experimental animals: State details } \\
\text { of authority granting ethics approval (IRB or } \\
\text { equivalent committee(s), provide reference number } \\
\text { for approval. }\end{array}$ & & $\mathrm{x}$ \\
\hline $\begin{array}{l}\text { Studies involving specimen and field samples: State if } \\
\text { relevant permits obtained, provide details of } \\
\text { authority approving study; if none were required, } \\
\text { explain why. }\end{array}$ & & $\mathrm{x}$ \\
\hline
\end{tabular}

\begin{tabular}{|l|c|c|}
\hline Dual Use Research of Concern (DURC) & Yes (indicate where provided: page no/section/legend) & n/a \\
\hline $\begin{array}{l}\text { If study is subject to dual use research of concern, } \\
\text { state the authority granting approval and reference } \\
\text { number for the regulatory approval }\end{array}$ & & $\mathrm{x}$ \\
\hline
\end{tabular}




\section{Analysis}

\begin{tabular}{|l|l|l|}
\hline Attrition & Yes (indicate where provided: page no/section/legend) & n/a \\
\hline $\begin{array}{l}\text { State if sample or data point from the analysis is } \\
\text { excluded, and whether the criteria for exclusion were } \\
\text { determined and specified in advance. }\end{array}$ & $\begin{array}{l}\text { Tables S3 }-\mathrm{S} 4 \\
\text { Materials and Methods: lines } 335-340\end{array}$ & \\
\hline
\end{tabular}

\begin{tabular}{|l|l|l|}
\hline Statistics & Yes (indicate where provided: page no/section/legend) & n/a \\
\hline $\begin{array}{l}\text { Describe statistical tests used and justify choice of } \\
\text { tests. }\end{array}$ & Materials and Methods: lines 377-385 & \\
\hline
\end{tabular}

\begin{tabular}{|l|c|c|}
\hline Data Availability & Yes (indicate where provided: page no/section/legend) & n/a \\
\hline $\begin{array}{l}\text { State whether newly created datasets are available, } \\
\text { including protocols for access or restriction on } \\
\text { access. }\end{array}$ & References: line 224 & \\
\hline $\begin{array}{l}\text { If data are publicly available, provide accession } \\
\text { number in repository or DOI or URL. }\end{array}$ & & $\mathrm{x}$ \\
\hline $\begin{array}{l}\text { If publicly available data are reused, provide } \\
\text { accession number in repository or DOI or URL, where } \\
\text { possible. }\end{array}$ & & $\mathrm{x}$ \\
\hline
\end{tabular}

\begin{tabular}{|l|l|c|}
\hline Code Availability & Yes (indicate where provided: page no/section/legend) & $\mathrm{n} / \mathrm{a}$ \\
\hline $\begin{array}{l}\text { For all newly generated code and software essential } \\
\text { for replicating the main findings of the study: }\end{array}$ & & \\
\hline State whether the code or software is available. & & $\mathrm{x}$ \\
\hline $\begin{array}{l}\text { If code is publicly available, provide accession } \\
\text { number in repository, or DOI or URL. }\end{array}$ & & $\mathrm{x}$ \\
\hline
\end{tabular}

\section{Reporting}

\begin{tabular}{|l|l|c|}
\hline Adherence to community standards & Yes (indicate where provided: page no/section/legend) & n/a \\
\hline $\begin{array}{l}\text { MDAR framework recommends adoption of } \\
\text { discipline-specific guidelines, established and } \\
\text { endorsed through community initiatives. Journals } \\
\text { have their own policy about requiring specific } \\
\text { guidelines and recommendations to complement }\end{array}$ & & \\
MDAR. & & \\
\hline $\begin{array}{l}\text { State if relevant guidelines (eg., ICMJE, MIBBI, } \\
\text { ARRIVE) have been followed, and whether a checklist } \\
\text { (eg., CONSORT, PRISMA, ARRIVE) is provided with } \\
\text { the manuscript. }\end{array}$ & & \\
\hline
\end{tabular}

